\title{
Open Mesoscopic Systems: beyond the Random Matrix Theory
}

\section{Dissertation}

zur Erlangung des Doktorgrades

der Mathematisch-Naturwissenschaftlichen Fakultäten der Georg-August-Universität zu Göttingen

vorgelegt von

\section{Alexandre Ossipov \\ aus Kemerovo/Rußland}

Göttingen 2003 
D7

Referent:

Koreferent:

Tag der mündlichen Prüfung:
Prof. Dr. Theo Geisel

Prof. Dr. Kurt Schönhammer

01.04 .2003 


\section{Contents}

1 Introduction $\quad 7$

1.1 Scattering matrix and related quantities .............. 8

1.2 Random matrix theory and its limitations . . . . . . . . . . . 11

2 Localization: one-dimensional Anderson model $\quad 15$

2.1 Localization in disordered systems . . . . . . . . . . . . . 16

2.2 Anderson model with one open channel . . . . . . . . . . . . . 16

2.3 From transfer matrix to Hamiltonian map . . . . . . . . . . . . . . 18

2.4 Distribution of phases . . . . . . . . . . . . . . . 20

2.5 Distribution of Wigner delay times . . . . . . . . . . . . . . 23

2.6 Distribution of resonance widths . . . . . . . . . . . . . 27

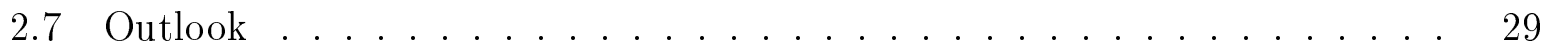

3 Diffusion: chaotic and disordered systems 31

3.1 Diffusion in chaotic and disordered systems . . . . . . . . . . . 32

3.1.1 From classical to quantum diffusion . . . . . . . . . . . 32

3.1.2 Quantum chaos and periodically driven systems . . . . . . . . 32

3.2 Closed systems: distribution of eigenfunction intensities . . . . . . . . 34

3.2.1 One-dimensional and two-dimensional kicked rotor models . . . . . 34 
3.2 .2 One-dimensional kicked rotor . . . . . . . . . . . . 38

3.2.3 Two-dimensional kicked rotor . . . . . . . . . . . . . . 41

3.3 Open systems: distribution of resonances and delay times . . . . . . . . 46

3.3.1 Open kicked rotor model . . . . . . . . . . . . . 46

3.3.2 Resonance widths distribution . . . . . . . . . . . 47

3.3.3 Wigner delay times distribution . . . . . . . . . . . 52

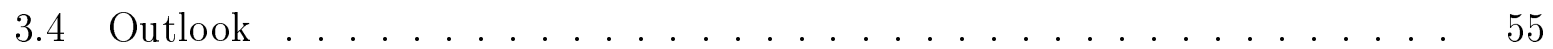

4 Criticality: one-dimensional quasiperiodic systems $\quad 57$

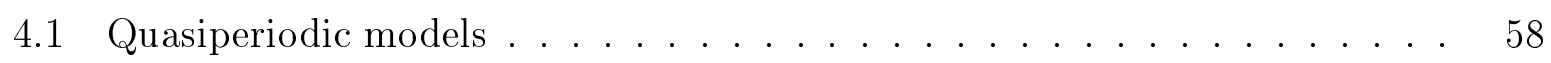

4.1 .1 Harper model . . . . . . . . . . . . . . . 58

4.1.2 Quasiperiodic tight-binding Hamiltonians . . . . . . . . . . . 60

4.1.3 Properties of the Harper and Fibonacci chain models . . . . . . . . 61

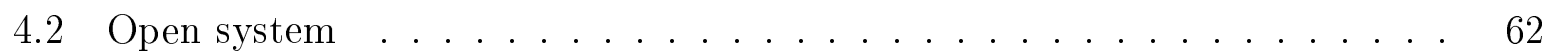

4.3 Statistics of resonances . . . . . . . . . . . . . . . . 64

4.4 Wigner delay times . . . . . . . . . . . . . . 66

4.5 Survival probability . . . . . . . . . . . . . . . 69

4.6 S-matrix autocorrelation function ..................... 75

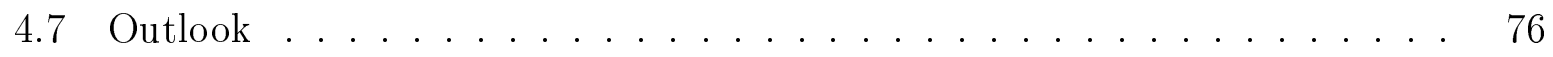

$\begin{array}{llr}5 & \text { Conclusion } & 79\end{array}$

A Effective Hamiltonian, resonances and Wigner delay time 81

A.1 Effective Hamiltonian for open one-dimensional system . . . . . . . . . 81

A.2 Wigner delay time and poles of the S-matrix . . . . . . . 83

B Single parameter scaling hypothesis $\quad 85$ 
C From Hamiltonian map to Fokker-Plank equation $\quad 87$

C.1 Expression for phases in terms of Hamiltonian map . . . . . . . . . . 87

C.2 Recursion relation for phases . . . . . . . . . . . . . . 88

C.3 Recursion relation for Wigner delay times . . . . . . . . . . 88

C.4 Fokker-Planck equation for distribution of Wigner delay times . . . . . . 89

D Mean free path and diffusion coefficient for the kicked rotor model $\quad 91$

D.1 Calculation of the mean free path . . . . . . . . . . . . . 91

D.2 Diffusion coefficient in the random phase approximation . . . . . . . . . 94

$\begin{array}{ll}\text { E Construction of the } S \text {-matrix for the kicked rotor model } & 97\end{array}$

E.1 Unitarity of the $S$-matrix $\ldots \ldots \ldots \ldots \ldots \ldots$

E.2 Wigner-Smith operator . . . . . . . . . . . . . 98 


\section{Chapter 1}

\section{Introduction}

Physical systems having sizes between microscopic and macroscopic are referred to as mesoscopic $[1,2]$. The motion in mesoscopic systems is phase-coherent, that means they must be treated by quantum mechanics. Another important property of mesoscopic systems is that they usually contain a lot of microscopic details (e.g. an impurity arrangement) which can not be taken into account exactly. Therefore one chooses a statistical approach considering an ensemble of systems having different microscopic configurations but the same macroscopic parameters. The rapid development of technology of fabrication of small electronic structures, having dimensions from a few nanometers to hundreds of microns, allows now to study mesoscopic systems experimentally.

In experiments or measurements one deals not with idealized closed, but with open systems. The natural way to describe an open system in quantum mechanics is to use a scattering formalism. Statistical approach to the scattering problems in mesoscopic physics is usually based on the random matrix theory (RMT) [3,4]. The strength of the RMT consists in the universality of its predictions containing no energy or length scales or any parameter dependence. At the same time this is the weakness of the RMT, because it does not allow to take into account various phenomena appearing in mesoscopic systems, which introduce new scales or parameters in the system, like for example localization.

This thesis is devoted to study exactly that type of scattering problems where "naive" RMT can not be applied. We consider different models of disordered and chaotic systems whose closed analogs have various specific features such as diffusion, fractality or localization. The investigation of different quantities related to scattering, like distributions of the Wigner delay times and the resonance widths, the survival probability, allows us to show how these features manifest themselves in the statistical properties of open systems. 


\subsection{Scattering matrix and related quantities}

Attaching leads to the sample makes it an open system. Then the whole system "sample+leads" can be described by a scattering setup (see Fig. 1.1). Taking into account that the potential $V$ vanishes inside the leads, the stationary Schrödinger equation there takes the following form:

$$
-\frac{\hbar^{2}}{2 m}\left(\frac{\partial^{2}}{\partial x^{2}}+\frac{\partial^{2}}{\partial x^{2}}\right) \psi(x, y)=E \psi(x, y)
$$

Denoting by $w$ the width of the leads and imposing the Dirichlet boundary condition at $y= \pm w / 2$ i.e. $\psi(x, \pm w / 2)=0$ one can represent the solution of Eq. (1.1) as a superposition of plane waves:

$$
\psi_{n}(x, y)=\left(\frac{m}{\pi \hbar^{2} k_{n}^{x} w}\right)^{\frac{1}{2}}\left[A_{n} e^{-i k_{n}^{x} x}+B_{n} e^{i k_{n}^{x} x}\right] \sin \left[k_{n}^{y}\left(y+\frac{w}{2}\right)\right],
$$

where $k_{n}^{x}=n \pi / w, k_{n}^{x 2}+k_{n}^{y^{2}}=k^{2} \equiv 2 m E / \hbar^{2}$. The index $n$ takes integer values $n=1 \cdots M$, where $M$ is the number of open channels, defined as the largest integer number less or equal to $k w / \pi$.

The Hamiltonian of the whole system is the sum of the Hamiltonian of the closed sample described by the $N \times N$ Hermitian matrix $H_{i n}$, the free Hamiltonian of the leads which corresponds to Eq. (1.1), and the coupling between sample and leads. Assuming that this coupling is local and the sample is finite we can argue that an asymptotic solution $(x \rightarrow \pm \infty)$ of the stationary Schrödinger equation for the whole system is given by the superposition of plane waves introduced in Eq. (1.2), where we denote by $A_{n}^{L(R)}$ and $B_{n}^{L(R)}$ the corresponding amplitudes for the left (right) lead.

According to the general definition, the scattering matrix $S$ relates the outgoing wave amplitudes to the incoming wave amplitudes. In our setup it is a $2 M \times 2 M$ matrix defined as

$$
\left(\begin{array}{l}
\mathbf{A}^{\mathbf{L}} \\
\mathbf{B}^{\mathbf{R}}
\end{array}\right)=S(E)\left(\begin{array}{c}
\mathbf{B}^{\mathbf{L}} \\
\mathbf{A}^{\mathbf{R}}
\end{array}\right)
$$

where $\mathbf{A}^{\mathbf{L}(\mathbf{R})}=\left(A_{1}^{L(R)}, \cdot, A_{M}^{L(R)}\right)^{T}$ and $\mathbf{B}^{\mathbf{L}(\mathbf{R})}=\left(B_{1}^{L(R)}, \cdot, B_{M}^{L(R)}\right)^{T}$. The flux conservation guarantees that the $S$-matrix is unitary

$$
S S^{\dagger}=I
$$

and can be written in a block-diagonal form:

$$
S=\left(\begin{array}{ll}
r & t^{\prime} \\
t & r^{\prime}
\end{array}\right)
$$




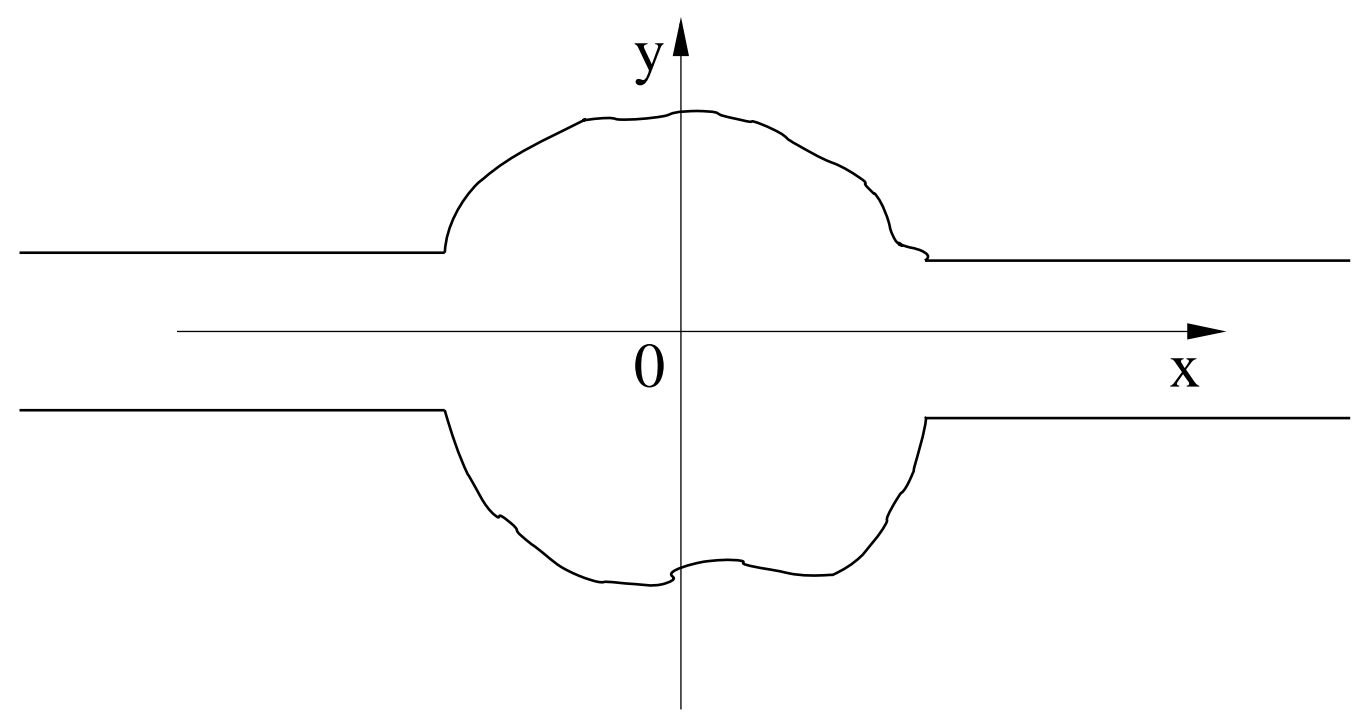

Figure 1.1: Two-dimensional sample attached to two leads.

All blocks in this decomposition have dimension $M \times M$. The matrix $r\left(r^{\prime}\right)$ describes the reflection for the waves coming from the left (right) lead, whereas the matrix $t\left(t^{\prime}\right)$ describes the transmission for the waves coming from the left (right) lead. This form of the $S$-matrix allows to connect its properties with quantities measurable in an experiment. Indeed, according to the Landauer formula $[5,6]$ the conductance $G$ of the mesoscopic sample can be calculated from the total transmission:

$$
G=\frac{2 e^{2}}{h} \operatorname{tr} t t^{\dagger}
$$

Thus one can extract the information about the conductance from the knowledge of the properties of the $S$-matrix. In this way it is possible to calculate for example the weak localization corrections to the conductance or the universal conductance fluctuations (for a review see $[4])$.

One can show $[7,8]$ that the scattering matrix can be written in the form (see also Appendix A)

$$
S=I-2 i \pi W^{\dagger} \frac{1}{E-H_{e f f}} W
$$

where the effective Hamiltonian $H_{\text {eff }}$ is given by

$$
H_{\text {eff }}=H_{i n}-i \pi W W^{\dagger} \text {. }
$$

The matrix $W$ is a rectangular $N \times 2 M$ matrix, which describes the coupling between sample and leads and generally is energy dependent. From Eq.(1.7) we see that the eigenvalues of the effective Hamiltonian $H_{e f f}$ are the poles of the scattering matrix. Since the effective Hamiltonian given by Eq. (1.8) is non-Hermitian its eigenvalues are complex $\mathcal{E}=E-\frac{i}{2} \Gamma$. 
The poles of the $S$-matrix correspond to the resonances - long-lived quasi-stationary states leaving in the interaction region [9]. Because of the presence of negative imaginary part of the eigenvalue, the resonant states decay in time exponentially. The quantity $\Gamma$ is referred to as the resonance width and it is inverse proportional to the lifetime of the corresponding resonant state. The properties of resonances are not only fundamental for the dynamics of the particle inside an open mesoscopic system (see Eq. (1.11 bellow), they are also directly related to the quantities measurable in experiments. One example is a quantum dot in the regime of Coulomb blockade. It is known that conductance in this case can be expressed in terms of the resonances [10,11]. Another application is related to the study of random lasers $[12,13]$. In this field one considers a multiple scattering of light in some disordered and in the same time amplifying media. The existence of the amplification leads to the shift of the poles of the $S$-matrix toward the real axis. When one of the poles reaches the real axis the lasing instability takes place: the gain due to the amplification becomes larger than the decay caused by opening of the system. Thus the position of the resonances are important for the understanding of the lasing instability.

The knowledge of the $S$-matrix allows in principle to study all characteristics of scattering problem. One of these characteristics is the time spent by the particle in the scattering region. Whereas in classical physics this issue is well defined without any additional explanations, in quantum mechanics one should first give a correct definition of this quantity. This problem is a fundamental one, since there is no natural quantum observable associated with time, and has a long history, which goes back to the works of Wigner and Eisenbud $[14,15]$. The idea that they used is to follow the peak of the scattered wave packet. Then one can define the Wigner delay time $\tau$ as a delay in propagation of the peak due to the interaction with the scatterer in comparison with a free propagation. Mathematically the Wigner delay time can be derived from the $S$-matrix as follows $[8,15,16]$. Since the $S$-matrix is unitary its eigenvalues have form $\exp \left(i \theta_{n}\right)$ with some real phases $\theta_{n}$. Then the Wigner delay time is given by the derivative of the total phase $\theta=\sum_{n} \theta_{n}$ with respect to energy. Another way to write it is

$$
\tau(E)=-i \hbar \frac{\partial}{\partial E} \ln \operatorname{Det} S=i \hbar \operatorname{Tr} \frac{\partial S^{\dagger}}{\partial E} S
$$

The Wigner-Smith time delay matrix $Q=i \hbar \frac{\partial S^{\dagger}}{\partial E} S$ introduced by Smith [17] generalizes the notion of the Wigner delay time. Its eigenvalues $\tau_{n}$, the so-called proper delay times, correspond to the delay time at one particular channel $n$. For the mesoscopic conductors the Wigner-Smith delay matrix determines the frequency-dependent current fluctuations [18-20]. In particular the admittance of the mesoscopic capacitor is given to leading order in frequency by [21]

$$
G^{I}(\omega)=-i \omega C_{e} \frac{\tau}{\tau+\eta}
$$

where $C_{e}$ is the geometric capacitance, $\tau$ is the dimensionless Wigner delay time, $\eta=$ $C_{e} \Delta /\left(M e^{2}\right)$, and $\Delta$ is the mean level spacing. The knowledge of the delay times makes also possible to understand the properties of the reflection eigenvalues $R_{n}$ of $S S^{\dagger}$ for optical or 
microwave cavities with absorption [22]. For the case of the weak absorption the eigenvalues $R_{n}$ can be expressed in a very simple way through the proper delay times $\tau_{n}: R_{n}=1-\tau_{n} / \tau_{a}$, where $1 / \tau_{a}$ is the absorption rate.

Another important quantity, which captures the time-dependent aspects of the scattering is the survival probability $P(t)$. It is defined as the probability of finding a particle inside the sample at time $t$ provided that the particle was injected into the sample at time $t=0$. Averaged over initial states it can be written as

$$
P(t)=\frac{1}{N} \sum_{i, k}\left\langle L_{k} \mid L_{i}\right\rangle\left\langle R_{i} \mid R_{k}\right\rangle \exp \left[i\left(\mathcal{E}_{i}^{*}-\mathcal{E}_{k}\right) t\right],
$$

with $\left|R_{k}\right\rangle$ and $\left\langle L_{k}\right|$ being the right and left eigenvectors and $\mathcal{E}_{k}$ the corresponding eigenvalues of the effective Hamiltonian $H_{\text {eff }}$. The survival probability is directly related to the problem of current relaxation in a mesoscopic sample [23], since it is proportional to the time-dependent conductance $G(t)$ defined by the Ohm law

$$
I(t)=\int_{-\infty}^{t} d t^{\prime} G\left(t-t^{\prime}\right) V\left(t^{\prime}\right)
$$

It is worth to mention that in mesoscopic systems, because of the strong fluctuation of the quantities discussed above (even in the thermodynamic limit), one needs to study not only the average values, but the whole statistical distributions.

\subsection{Random matrix theory and its limitations}

The properties of the scattering matrix strongly depend on the character of the corresponding closed system. This can be clearly seen from Eq. (1.7) and Eq. (1.8), which give the connection between the $S$-matrix and the Hamiltonian $H_{i n}$ of the closed system. So it is natural to begin the analysis of the scattering problem by defining the properties of the closed sample.

As it was mentioned above mesoscopic samples with the same macroscopic parameters can differ in microscopic details like disorder configurations or the exact shape of the boundaries. This force us to consider the corresponding Hamiltonian as random one and leads to the statistical description of the problem. The simplest and at first glance naive way to implement the idea of some randomness of the Hamiltonian is to assume that each of its matrix element is independent random number with some (for example Gaussian) probability distribution. However it turns out that this idea, which is underlying for the so-called random matrix theory (RMT) [3], is very fruitful and efficient not only in the field of disordered mesoscopic systems, but also in many other areas of physics. 
The RMT was pioneered by Wigner [24], who proposed to use large random matrices as Hamiltonians of complex nuclei in order to describe their excitation spectra. In 1960s the RMT was developed further by Dyson and Mehta [3,25]. The starting point of RMT is the ensemble of random Hamiltonians determined solely by the global symmetries of the system. For example, if there are no special symmetries in the system, one introduces a set of all possible Hermitian matrices. For any unitary transformation this set is an invariant one. Supplied with some invariant measure described below it is referred to as Gaussian Unitary Ensemble (GUE). If the system obeys time-reversal symmetry, then one demands that the matrices should be real and symmetric. The invariant transformations are now orthogonal and the corresponding ensemble is referred to as Gaussian Orthogonal Ensemble (GOE). The invariant measure is given for both classes by

$$
P(H)=\exp \left(-a \operatorname{tr} H^{2}+b \operatorname{tr} H+c\right)
$$

with certain constants $a, b, c$. Using these probability distribution functions one can calculate all possible statistical properties of the eigenvalues and eigenfunctions of the random Hamiltonians. Since the probability measure (1.13) does not contain any system-specific information and depends only on the global symmetries, all predictions of the RMT are universal.

The success of the RMT is not restricted by nuclear physics only. In 1984 Bohigas et al. [26] put forward a conjecture that the statistical properties of spectra of "quantum chaotic" systems can be also described by the RMT. By a quantum chaotic system [27-29] one understands a quantum system whose classical analog is chaotic. It means that the dynamics of the system is exponential sensitive to the infinitesimal changes of the initial conditions. Therefore despite of the absence of any stochastic noise or disorder this type of systems shows very irregular behavior and "randomness" appears in this case from the deterministic dynamics. A lot of numerical results confirm the validity of the RMT for fully chaotic system. Nevertheless the justification of its applicability remains a conjecture, although some attempts were made in order to prove it analytically [30-33].

For disordered systems in the ballistic regime the RMT conjecture suggested first by Gor'kov and Eliashberg [34] was proven in 1982 by Efetov [35] using the so-called nonlinear supermatrix $\sigma$-model. Using Grassmann variables this approach allows to perform an averaging over the disorder potential explicitly mapping the problem to some effective field-theoretical model. In the limit when the spatial variations of the effective field can be neglected one can show [36] that the nonlinear $\sigma$-model reproduces the RMT results. The last condition corresponds to the limit, when the dimensionless conductance of the sample goes to infinity.

In the cases, where the random matrix theory is established as an appropriate tool for the description of a disordered or chaotic system, it is natural using Eq. (1.7) and Eq. (1.8) to apply it for the study of its open counterpart. In particular, one can ask, what are the distributions of resonance widths and delay times for the system, whose closed analogs 
obey the RMT statistics. This issue has been a subject of intensive research activity in the last years and many interesting results were obtained in this direction (for review see [8]).

As it was mentioned above, the main advantage of the RMT is its universality. In the same time the universality means, that the RMT doesn't "know" anything about the specific properties of the system under study. Since no physical parameters (except the global symmetries) are plugged in the RMT machinery, it is clear that it can give the correct predictions only in some limiting case, when all physical parameters and scales can be considered as irrelevant. This can be easily understood especially for the case of disordered systems, for which exact criteria of the applicability of the RMT exist.

One of the fascinated phenomenon known in disordered system is the phenomenon of localization. In 1958 Anderson [37] showed that a typical eigenstate of an one-dimensional tight-binding disordered model (known now as the Anderson model) is exponentially localized. The mechanism which is responsible for this result is the appearance of destructive quantum mechanical interference effects. One of the surprising consequences following from this fact is the prediction, that the conductance of an one-dimensional wire goes exponentially to zero with increasing its length and the system behaves as an insulator. Later the localization theory was developed not only for one-dimensional, but also for two- and three-dimensional cases (for review see [38]). According to the so-called scaling theory of localization the infinite one- and two-dimensional systems (in the absence of interaction) are always in the localized regime. For the three-dimensional case the situation is more subtle: if the disorder is sufficiently strong the eigenstates are again localized and the system has an insulating behavior, if the disorder is weak enough there is no localization and the system has a metallic behavior. This phenomenon is referred to as the Anderson metal-insulator transition. Exactly at the transition point the eigenfunctions exhibit multifractal properties with strong fluctuations on all scales and the system has a critical behavior.

The appearance of the localization and the corresponding length scale - the localization length (which is inverse proportional to the decay rate of the typical eigenfunction) make the applicability of the RMT of course impossible. What are the consequences of localization for open disordered system? This issue is studied in Chapter 2, where the distribution of the phases of the $S$-matrix and the Wigner delay times are found for one-dimensional Anderson model [39]. Because of the universality of the localization mechanism, it is natural to expect that the obtained results are valid in the localized regime independent from the dimensionality or the symmetry class.

The fact that the RMT is not valid in the localized regime is not surprising. But it is interesting that even in the metallic regime the application of the RMT is strongly restricted. Indeed, it is well known that the motion of the electrons in a metal is diffusive. The rate of the diffusion process is characterized by the diffusive constant, which according to the Einstein relation determines the conductance of the sample. This parameter is again not included in the random matrix theory, therefore its predictions are valid only in the 
limit when the conductance goes to infinity. What happens when the conductance is large but finite? One can suggest that in this situation it is possible to describe the deviations from the RMT theory in a perturbative way. This is however not completely true. It turns out that, despite the fact that in a metallic regime the localization length is much larger than the system size and typical eigenstates are extended, there are some states which are anomalously localized. They exist due to some rare disorder realizations and can be considered as the signature of the onset of localization in a metallic regime. Although these states are rare and untypical in some sense, their existence lead to the long tails, which deviate strongly from the predictions of the RMT, in the distribution of many physical quantities (see review [23]).

The phenomenon of localization is known not only for disordered systems but also in the field of quantum chaos. The prominent example is the dynamical localization [40] found for the kicked rotor model [41]. The eigenstates of system are exponentially localized like in the case of the Anderson localization, although no disorder is present. It is clear again, that the RMT is not applicable for this chaotic system in the localized regime. Taking into account the discussion of the previous paragraph, it is natural to ask, what happens with such a quantum chaotic system when the localization length exceeds the system size and the localization is not dominant. Do the anomalously localized states exist in this case? Do the distribution of the physical quantities deviate from the RMT prediction? These questions are addressed in Chapter 3.1, where the distribution of the eigenfunction intensities are studied for the kicked rotor model and its two-dimensional generalization [42]. After establishing the kicked rotor as a nice model to study the metallic regime in chaotic and disordered systems we come back to the main issue of this thesis, namely to the properties of open systems. Construction of the $S$-matrix for the two-dimensional kicked rotor in Chapter 3.2 allows us to investigate, how the classical diffusion on the one hand and the existence of the anomalously localized states on the other hand affect the distributions of the resonance widths and the Wigner delay times [43]. Based on the results of Chapter 3.1 one can claim that these results are valid not only for chaotic but also for disordered systems.

The criticality is also out of scope of the RMT. The Anderson metal-insulator transition is a very hard problem both for analytical and numerical study. One reason for this is the fact that one should deal with a high-dimensional problem. In order to avoid this problem one can study instead critical systems in lower dimensions. This is the subject of Chapter 4 . In this chapter the statistics of the resonance widths, the distribution of the Wigner delay times, the survival probability, and the $S$-matrix autocorrelation function are investigated for various one-dimensional quasi-periodic systems at critical conditions [44,45]. This class of systems is interesting by itself (see for example [46]). In particular, the one-dimensional Harper model describes effectively an electron in a two-dimensional periodic potential in a magnetic field.

Finally, Chapter 5 contains the summary of the main results of this thesis. 


\section{Chapter 2}

\section{Localization: one-dimensional Anderson model}

This chapter deals with one-dimensional tight-binding model with random potential. It is well known that eigenfunctions of the closed system are exponentially localized. Here we investigate the properties of the open system, in particular, we study the statistics of phases of the $S$-matrix and the Wigner delay times both analytically and numerically for different strength of disorder potential. The importance of the distribution of phases of $S$-matrix for the single parameter scaling hypothesis is discussed. We also analyze how the different type of disorder influences on the distribution of the Wigner delay times. 


\subsection{Localization in disordered systems}

The concept of localization was introduced in 1958 by Anderson [37]. He was the first who pointed out that quantum diffusion is suppressed in random media due to destructive interference effects. In particular, he found that the eigenfunctions of a disordered sample are exponentially localized, provided that the disorder is sufficiently strong:

$$
|\psi(\mathbf{r})| \sim \exp \left(\left|\mathbf{r}-\mathbf{r}_{0}\right| / \xi\right)
$$

where the parameter $\xi$ is referred to as localization length and shows the degree of localization. The appearance of localization can be understood easily in the case of very strong disorder. In this limit the eigenstates in zero order approximation are represented by the localized states located in the deep minima of the random potential. If one wants to go beyond the zero order approximation, one should consider the overlap between different states. The states which are located nearby in space have usually very different values of energy due to the random character of the potential. Whereas the states which are nearly degenerate in energy are usually located at very different points in space, so that their overlap is infinitesimal small. Thus there is no possibility to establish an existence of any extended state and the resulting eigenstate is exponentially localized.

It turns out that the eigenstates are exponentially localized in one dimensional systems even for arbitrary weak disorder and the corresponding localization length is of the order of the mean free path. This statement is rigorously proved now by different methods [47-49]. According to the scaling theory of localization (see Section B and review [38]) the same is true for two dimensions. The localization length in this case scales exponentially with the mean free path. In three dimensions the degree of localization depends on the strength of disorder. If the disorder is weak, there is no localization and the states are extended. For sufficiently strong disorder however all states are exponentially localized. The theory of localization is very important for many observable quantities like for example conductance. In particular, it predicts non Ohmic behavior for sufficiently large one- and two-dimensional samples and metal-insulator transition for three dimensional systems.

\subsection{Anderson model with one open channel}

One of the simplest and well-known models of disordered systems, which allows to study the effect of localization, is the tight-binding Anderson model. The tight-binding model is a useful approximation used not only in solid state physics [50], but also in optics and acoustics. Within this model the wavefunction is expressed in terms of localized atomic orbitals one at each site. In this representation the one-dimensional Schrödinger equation takes the form:

$$
\psi_{n+1}+\psi_{n-1}+V_{n} \psi_{n}=E \psi_{n}
$$


where $E$ is the dimensionless energy and $V_{n}$ is the on-site potential. The diagonal term of the tight-binding Hamiltonian describes the particle localized at some site $n$ with the eigenenergy $V_{n}$. Two non-diagonal terms allow the particle to hop from the site $n$ to the nearest neighbor sites $n-1$ and $n+1$. Eq.(2.2) can be thought also as a discrete version of the Schrödinger equation after one replaces the Laplace operator by the finite differences.

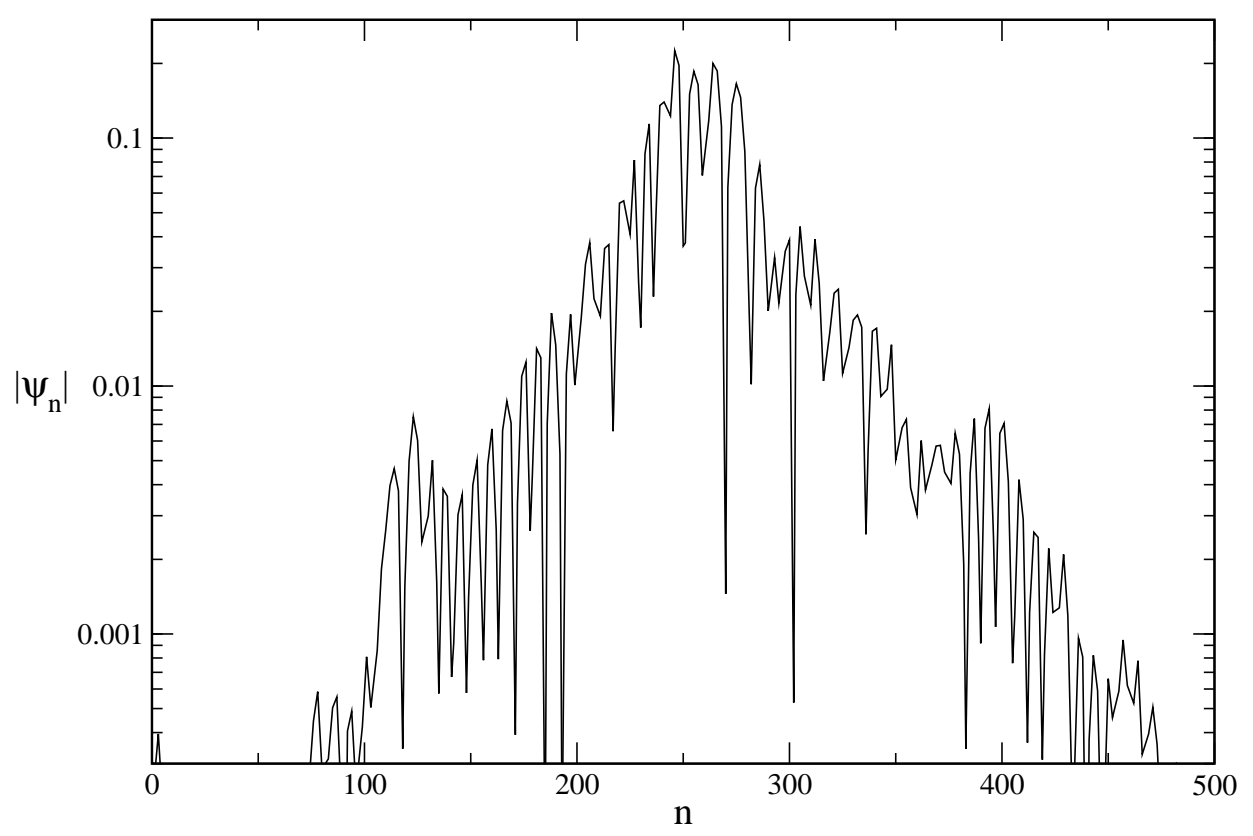

Figure 2.1: Example of the exponentially localized eigenstate of the one-dimensional Anderson model.

One dimensional disordered systems can be described by Eq. (2.2) when one considers the on-site potential $V_{n}$ as a random variable having some probability distribution. This model is referred to as the Anderson model. The strength of the disorder potential determines the energy mismatch between different sites, whereas the hopping elements (equal to one in our case) control the probability that a particle will be transfered to another site. Thus the important parameter is the ratio of the disorder strength to the hopping strength, which fixes the degree of localization in the system. In agreement to the general theory of localization the eigenstates of the Anderson model are exponentially localized (Fig. 2.1) for any disorder strength.

In the following we consider a finite disordered sample of length $L$ with one semi-infinite perfect lead attached on the left side (see Fig. 2.2). This means that $V_{n}$ are delta-correlated 
random variables with a distribution $\mathcal{P}_{V}$ having mean zero and variance $\sigma_{V}^{2}$ for $0 \leq n \leq L$. For $n<0, V_{n}=0$ and we impose Dirichlet boundary condition at the edge $\psi_{L+1}=0$.

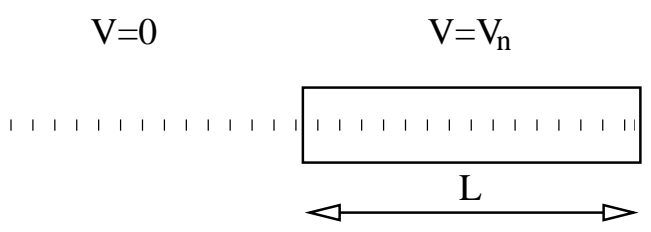

Figure 2.2: Disordered sample of size $L$ with one semi-infinite perfect lead attached on the left side.

The solution of Eq.(2.2) for $n \leq 0$ can be found easily:

$$
\begin{aligned}
\psi_{n} & =e^{i k n}+r e^{-i k n}, \quad n \leq 0 \\
E & =2 \cos k
\end{aligned}
$$

It represents a superposition of an incoming and a reflected plane waves. Since there is only backscattering, the reflection coefficient $r(E) \equiv e^{i \Phi(E)}$ is of unit modulus and the total information about the scattering is contained in the phase $\Phi(E)$. The Wigner delay time is given now according to its general definition $(1.9)$ by $\tau(E) \equiv \frac{d \Phi(E)}{d E}$ (here we put $\hbar=1$ ).

Our aim is to find the probability distributions of the phases $\mathcal{P}_{\Phi}(\Phi)$ and of delay times $\mathcal{P}_{\tau}(\tau)$ in the thermodynamic limit, i.e. when $L \rightarrow \infty$. The knowledge of the distribution of phases is not only necessary to calculate the distribution of delay times, which is in the focus of our main interest, but it has its own importance. The question, whether the phases are distributed uniformally or not, is crucial for the justification of the single parameter scaling hypothesis (see Appendix B), which is the cornerstone of the localization theory .

\subsection{From transfer matrix to Hamiltonian map}

Equation (2.2) can be written equivalently in a form

$$
\left(\begin{array}{c}
\psi_{n+1} \\
\psi_{n}
\end{array}\right)=M_{n}\left(\begin{array}{c}
\psi_{n} \\
\psi_{n-1}
\end{array}\right)
$$

where $M_{n}$ is a transfer matrix given by

$$
M_{n}=\left(\begin{array}{cc}
E-V_{n} & -1 \\
1 & 0
\end{array}\right)
$$

Equation (2.4) can be iterated so that one relates the wavefunction amplitudes at sites $n=-1$ and $n=0$, where the wavefunction is a superposition of two plane waves described 
by Eq. (2.3), with wavefunction amplitudes at sites $n=L$ and $n=L+1$. Recalling that $\psi_{L+1}=0$ due to the Dirichlet boundary condition, we can write the following equation:

$$
\left(\begin{array}{c}
0 \\
\psi_{L}
\end{array}\right)=\left(\begin{array}{ll}
P_{11} & P_{12} \\
P_{21} & P_{22}
\end{array}\right)\left(\begin{array}{c}
1+r \\
\mathrm{e}^{-i k}+r \mathrm{e}^{i k}
\end{array}\right),
$$

where matrix $P$ is a total transfer matrix given by the product of all transfer matrices at each site $P=\prod_{n=0}^{L} M_{n}$. From this equation $r$ can be found:

$$
r=-\frac{P_{11}+P_{12} \mathrm{e}^{-i k}}{P_{11}+P_{12} \mathrm{e}^{i k}}
$$

Introducing a new variable $\phi=\Phi / 2$ and noting that the nominator in the last expression is a complex conjugate to the denominator, one obtains (Appendix C.1):

$$
\tan \phi=\left(\frac{P_{11}+P_{12} \cos k}{P_{12} \sin k}\right) .
$$

As was indicated in Ref. [51,52], one can introduce new variable $\left(x_{n}, p_{n}\right)$ by

$$
\left(\begin{array}{c}
x_{n} \\
p_{n}
\end{array}\right)=R\left(\begin{array}{c}
\psi_{n} \\
\psi_{n-1}
\end{array}\right), \quad R=\left(\begin{array}{cc}
1 & 0 \\
\frac{\cos k}{\sin k} & \frac{-1}{\sin k}
\end{array}\right)
$$

so that Eq. (2.4) takes the form (Appendix C.1)

$$
\begin{array}{r}
\left(\begin{array}{c}
x_{n+1} \\
p_{n+1}
\end{array}\right)=Q_{n}\left(\begin{array}{c}
x_{n} \\
p_{n}
\end{array}\right), \\
Q_{n}=\left(\begin{array}{cc}
\cos k-A_{n} \sin k & \sin k \\
-A_{n} \cos k-\sin k & \cos k
\end{array}\right) .
\end{array}
$$

$Q_{n}$ is related to the transfer matrix $M_{n}$ through a similarity transformation $Q_{n}=R M_{n} R^{-1}$ and $A_{n}=\frac{V_{n}}{\sin k}$. If we consider $\left(x_{n}, p_{n}\right)$ as coordinates in a phase space, then Eq. $(2.10)$ describes the time evolution of a system with the Hamiltonian $H$ given by

$$
H=\frac{k p^{2}}{2}+\frac{k x^{2}}{2}-\frac{x^{2}}{2} \sum_{n} A_{n} \delta(t-n),
$$

which is a Hamiltonian of a parametric linear oscillator subjected to periodic kicks of strength $A_{n}$ and period $T=1$. Between two successive kicks, there is a free rotation in the phase space which is determined by the eigenenergy $E$ of our initial equation (2.2). In such a representation, the amplitudes $\psi_{n}$ of a specific eigenstate correspond to positions of the oscillator at times $t_{n}=n$.

The total transfer matrix $P$ is related to the map $F=\prod_{n=0}^{L} Q_{n}$ through the similarity transformation $P=R^{-1} F R$. Using this, together with (2.8) we write $\phi$ in terms of the Hamiltonian map $F$ as (Appendix C.1):

$$
\tan \phi=\left(\frac{F_{11}}{-F_{12}}\right) \text {. }
$$


We can give a geometrical interpretation for (2.12). Consider the time evolution of the vector $v(t=0)=(0,1)^{T}$ under the inverse map $F^{-1}$. For time $t_{n}=L$ we have

$$
v(t=L)=F^{-1}\left(\begin{array}{l}
0 \\
1
\end{array}\right)=\left(\begin{array}{c}
-F_{12} \\
F_{11}
\end{array}\right) .
$$

Then $\phi$ is exactly the angle between the vector $v(t=L)$ and the $\mathrm{x}$-axis. It is convenient therefore to pass to polar coordinates $\left(r_{n}, \theta_{n}\right)$, using the transformation $x=r \cos \theta$ and $p=r \sin \theta$. Then (2.10) is written in the form [51]:

$$
\begin{aligned}
r_{n+1}^{2} & =r_{n}^{2} D_{n} ; D_{n}^{2}=\left(1+A_{n}^{2} \cos ^{2} \theta_{n}-A_{n} \sin 2 \theta_{n}\right) \\
\cos \theta_{n+1} & =D_{n}^{-1}\left[\cos \left(\theta_{n}-k\right)-A_{n} \cos \theta_{n} \sin k\right] \\
\sin \theta_{n+1} & =D_{n}^{-1}\left[\sin \left(\theta_{n}-k\right)-A_{n} \cos \theta_{n} \cos k\right] .
\end{aligned}
$$

Inverting the last two equations for $n=L$ we obtain the following relation (Appendix C.2):

$$
\tan \phi_{L+1}=\tan \left(\phi_{L}+k\right)+A_{L+1},
$$

which relates half of the phase of the scattering matrix for the chain of size $L$ with the one for the chain of size $L+1$ obtained from the previous chain by adding a new site at $n=L+1$. Eq. (2.15) can be also obtained directly from the initial tight-binding equation (2.2) [53].

Equation (2.15) is a starting point in order to get analytical results on the distribution of phases of scattering matrix presented in the next section.

\subsection{Distribution of phases}

The recursion relation (2.15) can be used in order to get a Frobenius-Perron equation [54] for the distribution of phases in the thermodynamic limit. Since the random variables $\phi_{L}$ and $A_{L+1}$ are statistically independent we have

$$
\mathcal{P}_{\tan \phi_{L+1}}(y)=\int d s \mathcal{P}_{\tan \left(\phi_{L}+k\right)}(y-s) \mathcal{P}_{A}(s),
$$

where we introduced the corresponding distributions of each term of Eq. (2.15). Rewriting this equation in terms of the distribution of $\phi$ yields

$$
\mathcal{P}_{\tan \left(\phi_{L}\right)}(y)=\frac{\mathcal{P}_{\phi_{L}}(\arctan y)}{1+y^{2}}
$$

Assuming further that $\mathcal{P}_{\phi_{L}}$ goes to some stationary distribution $\mathcal{P}_{\phi}$ when $L \rightarrow \infty$, we obtain

$$
\frac{\mathcal{P}_{\phi}(\arctan y)}{1+y^{2}}=\int d s \frac{\mathcal{P}_{\phi}(\arctan (y-s)-k)}{1+(y-s)^{2}} \mathcal{P}_{A}(s) .
$$


We start our analysis by investigating first the limit $\sigma_{A}=\sigma_{V} / \sin k \ll 1$ which occurs when the disorder is week $\sigma_{V} \ll 1$ and $k$ is far from the band edge $(k \neq 0, \pi)$. In this limit we can approximate $\mathcal{P}_{A}(s)$ by a $\delta$-function. Then from (2.18) we have that

$$
\mathcal{P}_{\Phi}(\Phi)=\mathcal{P}_{\Phi}(\Phi-k),
$$

which for $k$ equals to irrational multiples of $\pi$, immediately leads to a uniform distribution

$$
\mathcal{P}_{\Phi}(\Phi)=\frac{1}{2 \pi} ; k / \pi \notin Q
$$

in agreement with previous numerical results $[55,56]$. We understand $(2.20)$ in the following way: For $\sigma_{A} \ll 1$, the particle travels long distances inside the sample and undergoes many scattering events which leads to randomization of the phase. Our numerical results reported in Fig. 2.3a are in perfect agreement with (2.20). We notice here that the same uniform distribution of the reflection phases in the weak disorder limit was found also for continuous disordered models [57-59].

The case of $k$ equals to rational values of $\pi$ requires a special treatment. For these cases the wavefunction in the absence of disorder is commensurate with the lattice. Thus one expects that the discreteness of the lattice will show up. For example, for $k=\pi / 2$ we obtain [52]

$$
\mathcal{P}_{\Phi}(\Phi)=\left(2 \mathcal{K}\left(\frac{1}{\sqrt{2}}\right) \sqrt{3+\cos (2 \Phi)}\right)^{-1} ; \quad k=\frac{\pi}{2},
$$

where $\mathcal{K}$ is the complete elliptic integral of the first kind. The above expression (2.21) describe well our numerical results presented in Figs. 2.3b.

The deviation from the uniform distribution occurs at the band edge as well, i.e, when $k \rightarrow 0, \pi$. For this case the distribution has the form [52]

$$
\mathcal{P}_{\Phi}(\Phi)=\frac{f(\Phi / 2)}{\sin ^{2}(\Phi / 2)} \int_{0}^{\Phi / 2} d x \frac{2 J}{f(x) \sin ^{2} x},
$$

where $f(x)=\exp \left(2 \frac{k^{2}}{\sigma^{2}}\left(\frac{1}{3} \cot ^{3} x+\cot x\right)\right)$ and $J$ is a normalization constant.

In the $\sigma_{A} \gg 1$ limit, we distinguish between two parts of the spectrum. Namely, $k$ near the band edges (i.e. $k \simeq 0, \pi)$ and far away from them. For the latter case and for $V_{n}$ distributed uniformly between $\left[-\frac{V}{2} ; \frac{V}{2}\right]$ one can derive an analytical expression for $\mathcal{P}_{\Phi}$ using Eq. (2.16) in the thermodynamic limit. In this limit it is easy to show that $\mathcal{P}_{\tan (\phi)}(y)$ is given in the first order in $1 / \sigma_{A}$ by the uniform distribution:

$$
\mathcal{P}_{\tan (\phi)}(y)=\left(\sqrt{1} 2 \sigma_{A}\right)^{-1} \Theta\left(\sqrt{3} \sigma_{A}-|y+\cot k|\right),
$$

where $\Theta(x)$ is the Heaviside function. The distribution of phases $\mathcal{P}_{\Phi}(\Phi)$ can be derived easily from (2.23):

$$
\mathcal{P}_{\Phi}(\Phi)=\frac{1}{2 \sqrt{3} \sigma_{A}} \frac{\Theta\left(\sqrt{3} \sigma_{A}-\left|\tan \left(\frac{\Phi}{2}\right)+\cot k\right|\right)}{\cos ^{2}\left(\frac{\Phi}{2}\right)} .
$$




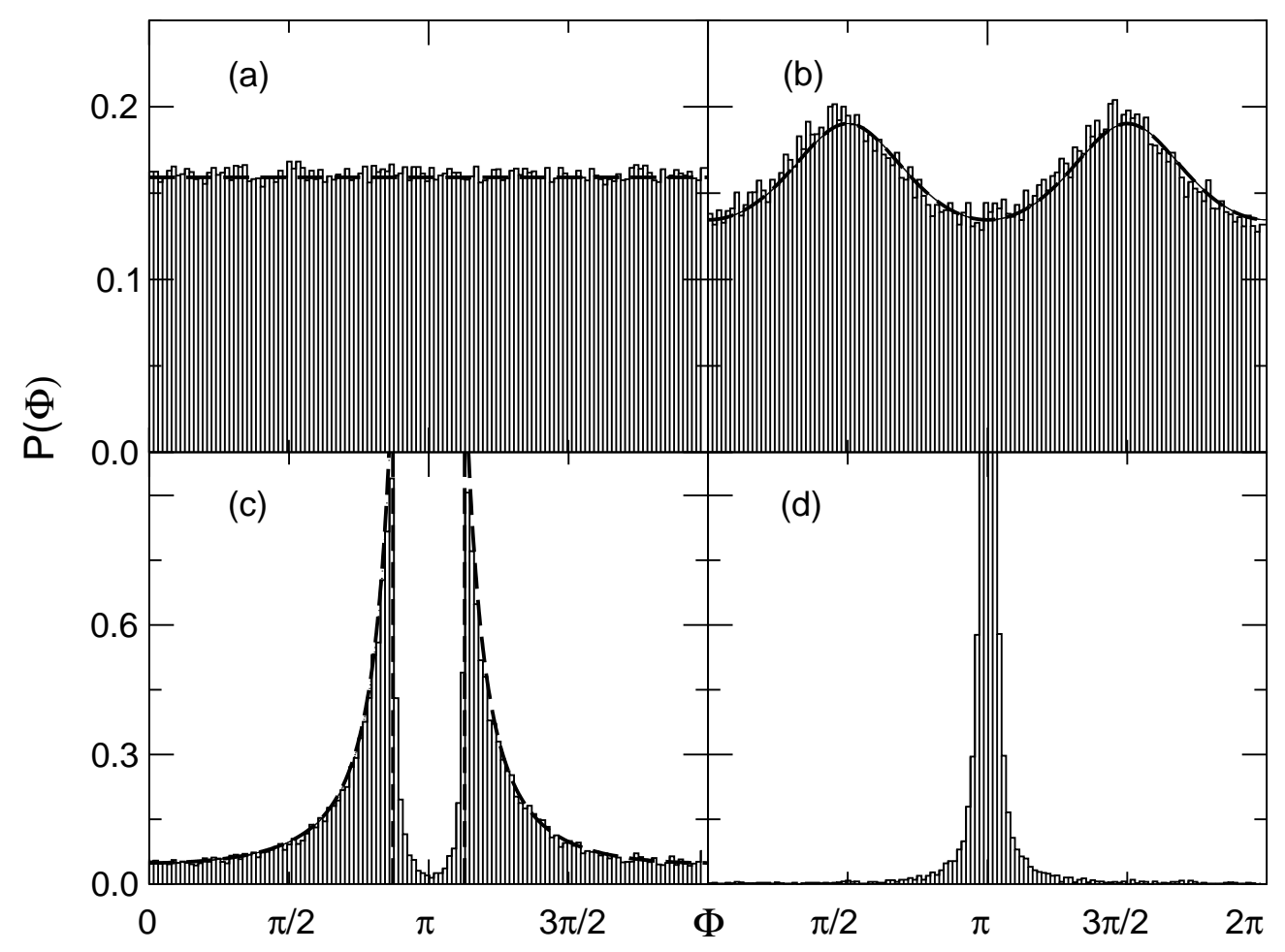

Figure 2.3: Distribution of the phases $\mathcal{P}(\Phi)$ for various disordered strengths and energies. The on-site potential is uniformly distributed between $\left[-\frac{V}{2} ; \frac{V}{2}\right]$. (a) $V=0.2$ and $k=\sqrt{\pi}$. (b) $V=0.2$ but now for $k=\pi / 2$ (band center); (c) $V=10$ and $k=\sqrt{\pi}$. Our numerical data (histogram) are in perfect agreement with the analytical predictions (dashed lines). (d) $V=0.2$ and $k=10^{-3} \sqrt{\pi}$

In contrast with the $\sigma_{A} \ll 1$ limit (2.20), here the distribution of phases (2.24) is highly non-uniform. By increasing the disorder strength $\sigma$, two peaks appear in the neighborhood of $\Phi=\pi$ (see Fig. 2.3c) while a gap is created between them. In general they are not symmetric because of the presence of the term $\cot k$ in argument of the Heaviside function Eq. (2.24). As $\sigma_{A}$ increases further, the two peaks move closer to one another. Now the scattering is so strong that most of the particles are reflected back from near the surface, thus being scattered only from a few sites. The possibility to be scattered from extremely strong on-site potential having either positive or negative sign explains the existence of two peaks in the distribution. In the limit $\sigma_{A} \rightarrow \infty$, the distribution becomes a $\delta$ function centered at $\Phi=\pi$. Eq. (2.24) is in very good agreement with our numerical data presented in Fig. 2.3c. The behavior near $\Phi=\pi$ is more subtle than the one given above; nevertheless, 
(2.24) gives the correct scale on which the distribution vanishes near $\Phi=\pi$. Distribution (2.24) agrees quite nicely also for other disorder potentials. It is worthing to point that a similar expression as (2.24) was found in [58] for continuous models in the limit of strong disorder.

Near the band edge $(k \simeq 0, \pi)$, our detail numerical analysis showed that $\mathcal{P}_{\Phi}(\Phi)$ is again highly non- uniform. Namely the distribution becomes narrower and centered (although with a slight asymmetry) at $\Phi=\pi$. We notice that such a choice of the parameters, can be realized even for weak disorder $\sigma \ll 1$. In Fig.2.3d we present a representative case corresponding to $k=10^{-3} \sqrt{\pi}$ and $\sigma=0.0577$. We point that the closer we are to the band edge the strongest is the singularity around $\Phi=\pi$. We also notice the different form of the distribution with respect to the previous case $\left(\sigma_{A} \gg 1\right.$ but away from the band edges).

All these results show that the distributions of phases $\mathcal{P}(\Phi)$ undergoes a transition from uniform $\left(\sigma_{A}<1\right)$ to non-uniform behavior $\left(\sigma_{A}>1\right)$, where $\sigma_{A}=\sigma_{V} / \sin k$. The latter limit can be achieved either by decreasing the disorder strength $\sigma$, or by taking $k \rightarrow 0, \pi$. In context of single parameter scaling hypothesis, it means that it can be violated even in the case of weak disorder $\sigma_{V} \ll 1$. In this limit, the states near the band edge $k \approx 0, \pi$ never obey the single parameter scaling since $\sigma_{A} \gg 1$. Thus the spectrum of the system is divided into two groups with different scaling properties, which coexist at the same disorder strength [60].

\subsection{Distribution of Wigner delay times}

Let us now turn to the analysis of delay times. Taking the derivative of both sides of Eq. (2.15) with respect to energy we get (see Appendix C.3) the following iteration relation for $\tau_{n}$ :

$$
\begin{aligned}
\tau_{L+1} & =G_{L}^{-1}\left(\tau_{L}+\frac{1}{\sin k}\right)-\frac{A_{L+1}}{1+\left(\tan \left(\phi_{L}-k\right)+A_{L+1}\right)^{2}} \frac{\cot k}{\sin k} \\
G_{L} & =1+A_{L+1} \sin \left(2\left(\phi_{L}+k\right)\right)+A_{L+1}^{2} \cos ^{2}\left(\phi_{L}+k\right),
\end{aligned}
$$

which proves to be very convenient for numerical calculations since it anticipates the numerical differentiation which is a rather unstable operation.

For $\sigma_{A} \ll 1$ and $k$ equals to irrational multiples of $\pi$, we obtain an analytical expression for $\mathcal{P}_{\tau}(\tau)$. To this end, we first derive from (2.15) and (2.25) a set of stochastic differential equations for the phases $\phi$ and rescaled delay times $\tilde{\tau}=\sigma_{V}^{2} \tau$ (up to $\sigma_{A}^{2}$ ), respectively 
(Appendix C.4):

$$
\begin{aligned}
& \frac{d \phi}{d L} \simeq k-\sigma_{A}^{2} \sin (\phi+k) \cos ^{3}(\phi+k)+\cos ^{2}(\phi+k) A \\
& \frac{d \tilde{\tau}}{d L} \simeq-\sigma_{A}^{2}\left(\tilde{\tau}\left(\cos ^{2}(\phi+k)-\sin ^{2} 2(\phi+k)\right)-\sin k\right)-\tilde{\tau} \sin 2(\phi+k) A
\end{aligned}
$$

This system of stochastic differential equations corresponds to the Fokker-Planck equation for the joint probability distribution of $\phi$ and $\tilde{\tau}$ [61]. Using the fact that $\phi$ follows the uniform distribution (see Eq. (2.20)) and assuming that the variables $\phi$ and $\tilde{\tau}$ are statistically independent, we obtain the Fokker-Planck equation for $\mathcal{P}_{\tilde{\tau}}(\tilde{\tau}, L)$ (Appendix C.4):

$$
\frac{\partial \mathcal{P}_{\tilde{\tau}}(\tilde{\tau}, L)}{\partial L}=\frac{\sigma_{A}^{2}}{4}\left[\frac{\partial}{\partial \tilde{\tau}}\left((\tilde{\tau}-4 \sin k) \mathcal{P}_{\tilde{\tau}}(\tilde{\tau}, L)\right)+\frac{\partial}{\partial \tilde{\tau}}\left(\tilde{\tau} \frac{\partial}{\partial \tilde{\tau}}\left(\tilde{\tau} \mathcal{P}_{\tilde{\tau}}(\tilde{\tau}, L)\right)\right)\right] .
$$

The resulting stationary distribution is obtained by setting $\frac{\partial \mathcal{P}_{\tilde{\tau}}}{\partial L}=0$ and has the form

$$
\mathcal{P}(\tau)=\frac{\xi}{v \tau^{2}} \exp (-\xi / v \tau) ; \quad \sigma_{A} \ll 1,
$$

where $\xi=2\left(4-E^{2}\right) / \sigma^{2}$ is the localization length and $v=|\partial E / \partial k|$ is the group velocity. We note that (2.28) is independent of the nature of the disorder; it only depends on its second moment through the localization length. (2.28) takes its maximum value at $\tau_{\max }=0.5 \xi \mathrm{v}$, indicating that the most probable trajectory that an electron travels (forth and back) before it scatters outside the sample is the mean free path $l_{M}=\xi / 4$. As $\tau \rightarrow \infty, \mathcal{P}(\tau)$ shows a long time tail which goes as $2 \tau_{\max } / \tau^{2}$. This leads to a logarithmic divergence of the average value of $\tau$, indicating the possibility of the particle traversing the infinite sample before being totally reflected. As was indicated in [62] (see also [63]) this is another manifestation of the fact that in the localized regime the conductance shows lognormal distribution due to the presence of Azbel resonances. Our numerical results (see Fig. 2.4) are in perfect agreement with (2.28). The same expression as (2.28) was obtained in [64] (see also $[62,65,66])$ using different approaches for continuous disordered models.

Finally we discuss the distribution of delay times $\mathcal{P}(\tau)$ for $\sigma_{A} \gg 1$. In this limit, the technique of the Fokker-Planck equation can not be applied. However our iteration relation (2.25) has proven very efficient for numerical investigations. In Fig. 2.5a we show the distribution of the delay times for a uniform and a Gaussian on-site potential distribution with the same variance $\sigma_{v}^{2}=10$. It is clear that the short time distribution differs considerably in the two cases and also deviate strongly from the theoretical prediction (2.28) for $\sigma_{A} \ll 1$. On the other hand, the distribution of large delay times, shows the same $1 / \tau^{2}$ behavior independent of the form of the disorder potential. The asymptotics of the distribution for large $\tau$ is presented in Fig. 2.5b, where we plot the integrated distribution $I(\gamma)=\int_{0}^{\gamma} P\left(\gamma^{\prime}\right) d \gamma^{\prime}$ of the inverse delay time $\gamma=1 / \tau$ in double logarithmic scale. To this end, we calculated $10^{7}$ delay times using the iteration relation (2.25). In both cases (Gaussian and uniform distribution) presented in Fig. 2.5b we collected at least $10^{4}$ delay 


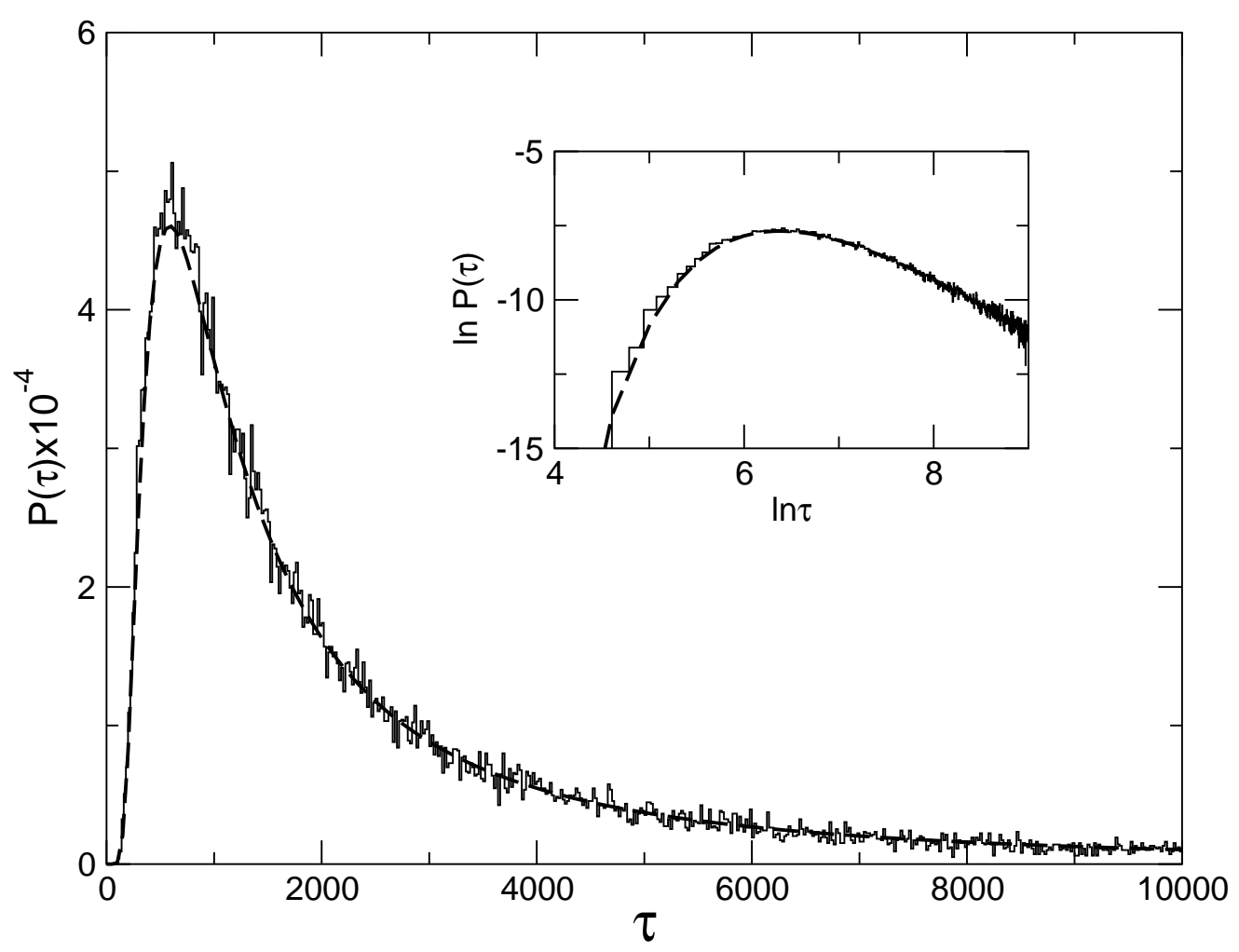

Figure 2.4: Distribution of the delay times $\mathcal{P}(\tau)$ for on-site potential, uniformly distributed between $[-0.1 ; 0.1]$ and wavenumber $k=\sqrt{\pi}$. The dashed line corresponds to $(2.28)$. In the inset we present the same data in a $\log -\log$ plot.

times that were larger than $\tau>150$. Our numerical data clearly show that $I(\gamma) \sim \gamma$ for $\gamma \ll 1$ and thus $\mathcal{P}(\tau) \sim 1 / \tau^{2}$. This disagrees with the results obtained for the continuous case in the low energy (strong disorder) limit [65]. In this case it was found that the distribution of delay times shows an exponential behavior which is in contradiction with our findings. How can one reconcile this numerical finding with the result of Texier and Comtet [65]? We give two possibilities: (a) One could argue that the discreteness of our model is responsible for this apparent contradiction. For this reason we calculated again $I(\gamma)$ for energy near the band edge corresponding to $k=\sqrt{\pi} 10^{-3}$, in order to minimize the influence of the discreteness. The disorder strength is $V=10$. Our results reported in Fig. 2.5b, in a $\log -\log$ scale, are based on more than $10^{4}$ delay times with $\gamma \ll 1$. Again a nice power-law behavior of the form $I(\gamma) \sim \gamma$ can be observed. However, one can not exclude a priori that we are still sensitive to the existence of the lattice. (b) The model 

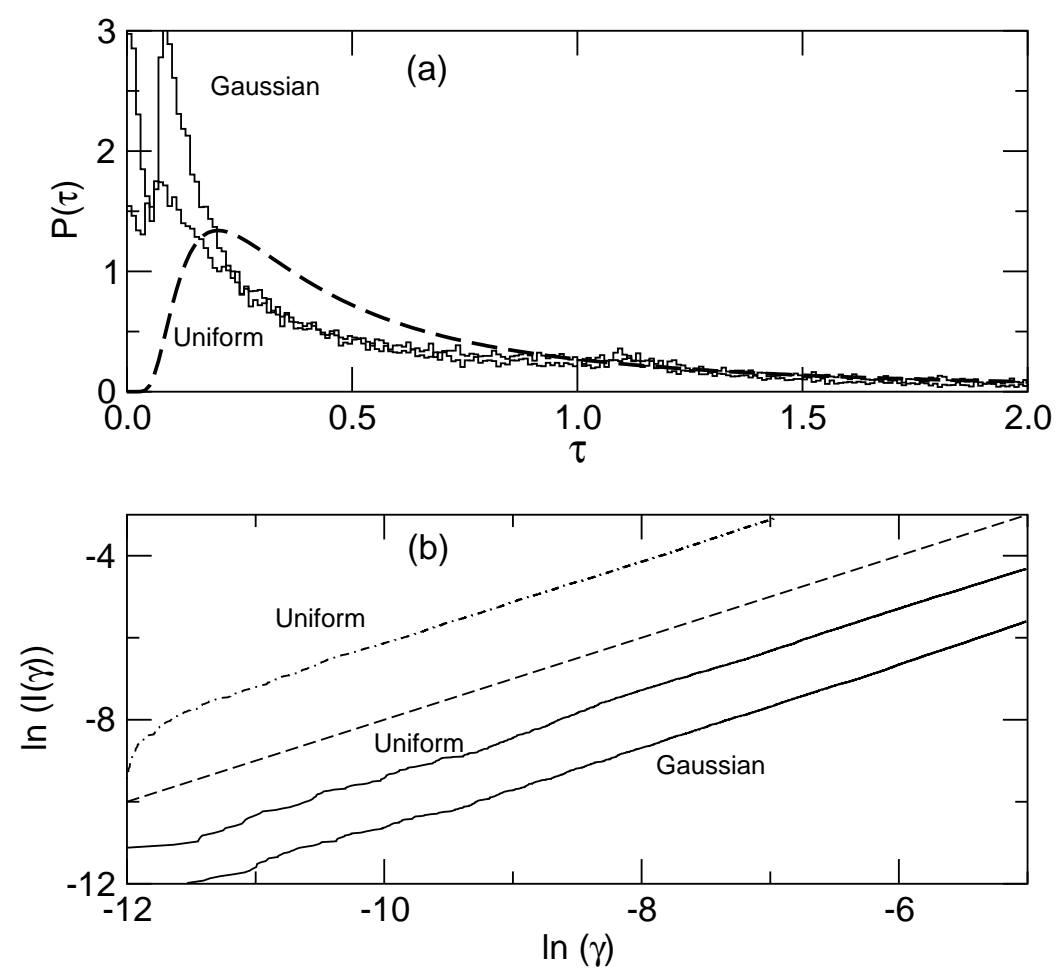

Figure 2.5: (a) Distribution of the delay times $\mathcal{P}(\tau)$ for uniform and Gaussian $\mathcal{P}_{V}$. In both cases the variance is $\sigma^{2}=10$ and the wavenumber is $k=\sqrt{\pi}$. The dashed line corresponds to (2.28). (b) The integrated distributions of inverse delay time $\gamma=1 / \tau$. Solid lines correspond to the same parameters as in (a) while the dot-dashed line correspond to the strong disorder case $(V=10)$ and wavenumber $k=\sqrt{\pi} 10^{-3}$ (band-edge). The dashed bold line has slope 1 and is drawn to guide the eye.

discussed in [65] differs with respect to ours in the way that the disorder is introduced; the weight of the $\delta$-scatterer are constant while their position is random (see also [55]). Recently Heinrichs [67] argued that $1 / \tau^{2}$ behavior can be found in the Anderson model by considering the scattering on the first site only, which is justified in the case of extremely strong disorder. 


\subsection{Distribution of resonance widths}

The resonances show up as the poles of the scattering matrix $S(\mathcal{E})$ in the complex plain. In the case of the one-dimensional system with one open channel this problem is reduced to the problem of finding poles of the reflection coefficient $r(\mathcal{E})$. This can be done by representing the reflection coefficient in terms of some effective Hamiltonian $H_{\text {eff }}$ and determination its eigenvalues in the complex plain (see Appendix A.1). The effective Hamiltonian $H_{e f f}$ is obtained from the Hamiltonian of the closed system by adding the imaginary shift $i$ to its first diagonal element (if one neglects its energy dependence at the band center):

$$
H_{e f f}=H-i \vec{e} \bigotimes \vec{e}
$$

where $\vec{e}=(1,0,0, \ldots, 0)^{T}$ is an $L$-dimensional vector, that describes at which site we open the system.

The behavior of the distribution of the resonance widths can be understood by the following consideration [68]. We know that the eigenstates of the closed system are exponentially localized. That means that the intensities of the eigenstates at the boundary are usually very small. For this reason most of the eigenstates are affected very weak once we open the system. Only the eigenstates localized near the boundary can "feel" the change of the boundary condition. However, when the system size becomes large, the probability of finding such eigenstates goes to zero. Thus we can consider the opening of the system as a small perturbation. Then taking into account the form of the effective Hamiltonian (2.29), we have in the first order perturbation theory:

$$
\Gamma_{k} \sim\left|\psi_{1}^{k}\right|^{2}
$$

where $\psi_{n}^{k}$ is the $n$-th component of an eigenstate of the closed system with energy $E_{k}$. This eigenstate is localized at some distance $l_{k}$ from the boundary. So we can estimate its intensity at the boundary as

$$
\left|\psi_{1}^{k}\right|^{2} \sim e^{-2 l_{k} / \xi}
$$

with $\xi$ being the localization length. This estimation yields

$$
\Gamma_{k} \sim e^{-2 l_{k} / \xi}
$$

Now assuming that the eigenstates are distributed uniformly along the system, i.e. the quantity $l_{k}$ is distributed uniformly in the interval $[0, L]$, we obtain the distribution of the resonance widths:

$$
\mathcal{P}(\Gamma) \sim \frac{1}{\Gamma}
$$

It is interesting to note, that in the thermodynamic limit the probability of finding an eigenstate at any finite distance from the boundary is equal to zero. Thus the distribution of the resonance widths in this case collapses into a delta function centered at zero. 


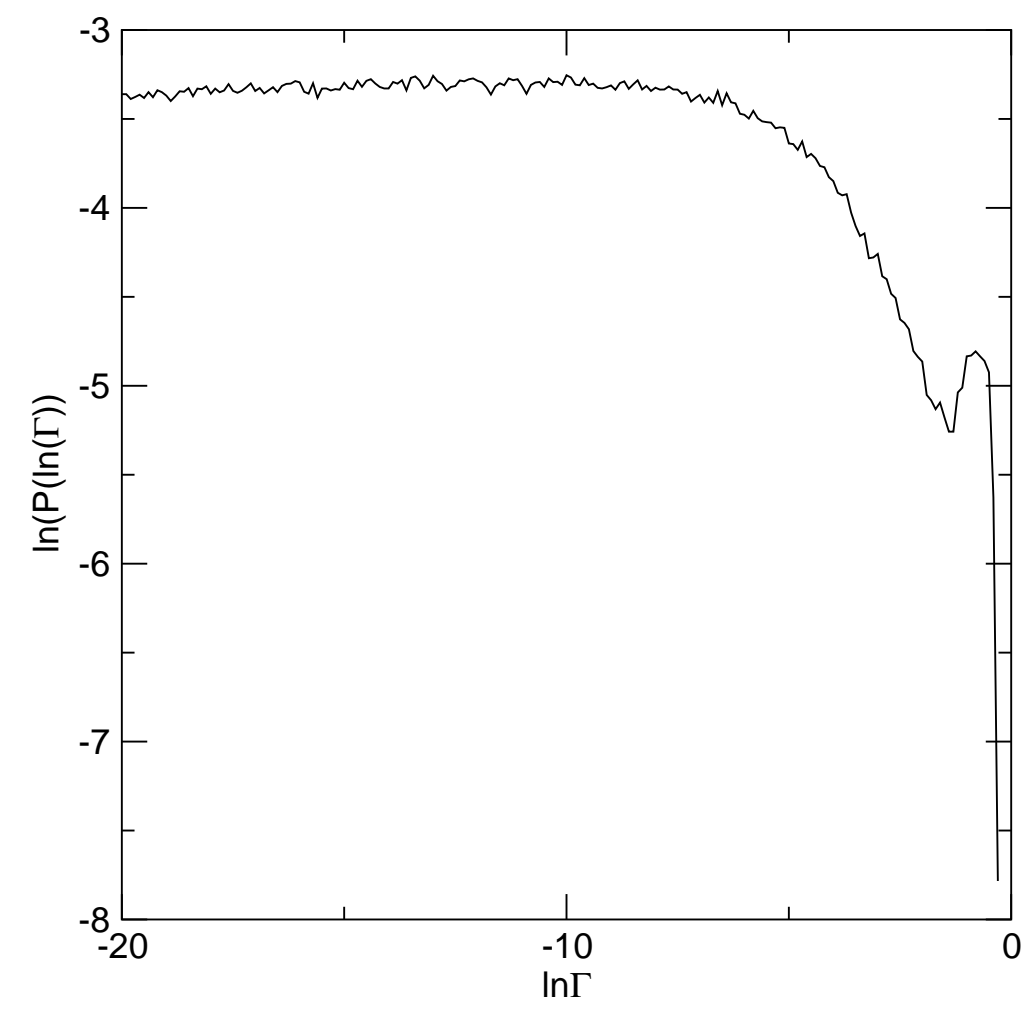

Figure 2.6: The distribution of $\ln \Gamma$ for the one-dimensional Anderson model.

We check the above prediction (2.33) by numerical diagonalization of the effective Hamiltonian (2.29). From Eq. (2.33) follows that $\mathcal{P}(\ln \Gamma)$ is constant, which is in nice agreement with numerical data (Fig. 2.6). Here we would like to mention that the distribution of the resonance widths was calculated analytically in Ref. [69]. According to this calculation the distribution is given by slightly different power law $P(\Gamma) \sim 1 / \Gamma^{1.25}$. However some steps of this calculation are uncontrollable. Moreover the later numerical calculation of Ref. [70] are in favor of the prediction of Eq. (2.33). 


\subsection{Outlook}

This chapter is concerned with distributions of phases of $S$-matrix and Wigner delay times in one-dimensional Anderson model with one open channel. It is shown that these distributions depend not only on the strength of the disorder $\sigma_{V}$ but on the wave vector $k$ of the incoming wave as well. Only in the case, when disorder is week and $k$ is far from the band edge and in the same time is not equal to a rational multiple of $\pi$, the distribution of phases is uniform. When one of this conditions is not satisfied the deviations from uniformity appear. This indicates a possible violation of the single parameter scaling hypothesis.

The universal form of the distribution of Wigner delay times is found analytically in the case when $\sigma_{A}=\sigma_{V} / \sin k \ll 1$. The important feature of this distribution is the power law $1 / \tau^{2}$ tail, leading to the divergence of the mean value of $\tau$ in the thermodynamic limit. This is explained in terms of Azbel resonances, which are some special realizations of disordered potential allowing the particle to penetrate arbitrary deep in the sample. In the opposite limit $\sigma_{A} \gg 1$ the origin of the distribution is model depended. However the tail of the distribution shows the same universal $1 / \tau^{2}$ behavior.

Finally the distribution of the resonance widths $P(\Gamma)$ is discussed. It is shown that it has power law behavior $P(\Gamma) \sim 1 / \Gamma$, which can be explained by a heuristic argument based on the exponential localization of the eigenstates.

It would be interesting to extend our investigation to systems in higher dimensions. Although the situation in the localized regime is expected to be the same independently of the dimensionality of the system, in higher dimensions one can study the full transition from the ballistic through the diffusive to the localized regime. The simplest geometry for which this question can be addressed is a geometry of the quasi-one-dimensional wire. It is known that the closed quasi-one-dimensional samples can be treated very efficiently by the nonlinear $\sigma$-model [35]. So it would be reasonable to try to apply the nonlinear $\sigma$-model to the open quasi-one-dimensional systems. 


\section{Chapter 3}

\section{Diffusion: chaotic and disordered systems}

This chapter deals with quantum disordered and chaotic systems in the diffusive regime. In this regime the underlying classical diffusion is very important for the behavior of many physical quantities. On the other hand, the quantum mechanical interference effects become crucial for the correct physical description of the systems. In particular, the existence of prelocalized states affects the statistical distribution of various quantities like for example the eigenfunction intensities. Whereas for disordered systems the field theoretical approaches were developed to describe the statistics of the eigenfunction intensities in this regime, nothing is known for dynamical systems with classical chaotic behavior. This issue is studied in the first part of this chapter. The second part is devoted to the properties of the corresponding open systems. We show what are the signatures of the classical diffusion and prelocalized states in the distribution of the resonance widths and delay times. 


\subsection{Diffusion in chaotic and disordered systems}

\subsubsection{From classical to quantum diffusion}

Diffusion process is a very general phenomenon which appears in many different areas of physics, chemistry and biology. In particular, the motion of electrons in a metal can be described classically by diffusion. The diffusion in a metal appears as a result of scattering of the electrons on the impurities, which is always present in non-ideal samples. The diffusion picture is sufficient to determine many important properties of the metals like for example its conductivity. Indeed according to the well known Einstein relation the conductivity $\sigma=e^{2}(\partial n / \partial \mu) D$ is proportional to the diffusion constant $D$.

One can think that diffusion appears only in stochastic systems. But this is not always the case. It can also appear in deterministic systems provided that they are chaotic. The chaotic systems are opposite to the integrable systems, where the motion is regular and can be described in a very simple way by an introduction of the appropriate action-angle variables. The motion in the chaotic systems is in contrast very irregular and complicated. In the limiting case of the fully developed chaos all trajectories are exponentially sensitive to any infinitesimal change of the initial conditions or perturbation [54,71].

In the area of the mesoscopic physics, where the motion is assumed to be phase-coherent, quantum mechanical effects become important. As was discussed already in Chapter 2, the diffusion in disordered systems can be affected very strongly by quantum localization. If the system size exceeds the localization length, then the diffusion is completely suppressed after the time needed for the wave packet to spread over the scale of the localization length. But even in the absence of strong localization the existence of prelocalized states [72-77] influences the behavior of different physical quantities in the diffusive regime.

Having this in mind, it is legitimate to ask, what happens with deterministic chaotic systems when quantum mechanical description becomes relevant? This issue is studied in the field of quantum chaos.

\subsubsection{Quantum chaos and periodically driven systems}

In quantum mechanics the uncertainly relation prevents a precise determination of the position in the phase space. Hence the notion of trajectory loses its meaning. Moreover the Schrödinger equation is a linear one, so one can not expect any exponential sensitivity to the change of the initial conditions. On the other hand the correspondence principle demands that quantum mechanics continuously develops into classical mechanics, as $\hbar \rightarrow 0$. Then the question arises, whether there are any criteria allowing to distinguish regular and chaotic systems in quantum mechanics? The study of quantum systems, whose classical 
analogs are chaotic, during last two decades shows that such criteria do exist [27-29].

One of these criteria is related to the energy spectra of chaotic systems. It is one of the cornerstones of quantum chaos and it emerged as a conjecture put forward by Bohigas et al. [26] in 1984. According to this conjecture the statistical properties of the energy spectra of chaotic systems are described by the random matrix theory [3, 24, 25], which was developed initially to study the spectra of complex nuclei. It was a very surprising discovery that the spectra of deterministic systems having only few degrees of freedom show the same behavior as those of complex many body systems. Despite the success of the random matrix theory in the quantum chaos its applicability is restricted only by fully chaotic systems in the ballistic regime. In particular the appearance of diffusion can not be captured by the RMT (see the discussion in Introduction).

A prominent example of a system with classical diffusion is, the well known in the field of quantum chaos, the kicked rotor model [41], which consists of the free propagating rotor driven periodically in time by the external force (see its detailed description in the next section). Since the total energy is not conserved for a driven system, the chaotic behavior can appear even in the one-dimensional case. For the same reason there is no stationary Schrödinger equation and one should consider a time dependent solution. Because of the periodicity of the external force a time dependent solution can be represented as a superposition of Floquet states [78], which are the eigenstates of the evolution operator for one period (Floquet operator). For the kicked systems the interaction with external force is instantaneous and one can factorize the total Floquet operator into the product of the evolution operators corresponding to the free propagation and the interaction. Due to this fact the kicked systems are very convenient for numerical study. Another advantage of the kicked rotor consists in the fact that due to the unitarity of the evolution operator all its eigenstates have the same statistical properties. This is in contrast to the eigenstates of Hamiltonian models (like Anderson model), where the eigenstates belonging to different parts of the spectrum have different statistical properties and therefore they must be picked up from a narrow energy window.

The kicked rotor model is also well known because of the phenomenon of the dynamical localization found by Fishman et al. in 1982 [79]. It turns out that the eigenstates of this model are exponentially localized similar to the eigenstates of disordered systems. Despite of the absence of disorder the localization occurs here due to the fact that chaotic dynamics creates complicated interference effects. Attempts were made to put on a solid base this analogy between the kicked rotor model and disordered models [31,79]. However all of them require an additional randomization or ensemble averaging. Therefore the problem remains open. In this context the study of the prelocalized states (known from disordered systems) for the kicked rotor model allows to test this analogy on a new level. This is one of the motivations of the investigation of the statistics of the eigenfunction of the kicked rotor in the diffusive regime presented in the next section. 


\subsection{Closed systems: distribution of eigenfunction in- tensities}

The statistical properties of wavefunction intensities have sparked a great deal of research activity in recent years. These studies are not only relevant for mesoscopic physics $[23,72-77,80-86]$, but also for understanding phenomena in areas of physics, ranging from nuclear [87] and atomic [88, 89] to microwave physics [90-93] and optics [94]. Experimentally, using microwave cavity technics it is possible to probe the microscopic structure of electromagnetic wave amplitudes in chaotic or disordered cavities [90-93]. Recently, the interest in this problem was renewed when new effective field theoretical techniques were developed for the study of the distribution of eigenfunction intensities $\mathcal{P}\left(|\psi|^{2}\right)$ of random Hamiltonians. As the disorder increases, these results predict that, the eigenfunctions become increasingly non-uniform, leading to an enhanced probability of finding anomalously large eigenfunction intensities in comparison with the random matrix theory prediction. Thus, the notion of prelocalized states has been introduced [72-77] to explain the appearance of long tails in the distributions of the conductance and other physical observables [72].

Up to now all theoretical predictions [23,72-77, 80-82] and numerical calculations [84-86] apply to disordered systems and are based on an ensemble averaging over disorder realizations. Their validity, however, for a quantum dynamical system (with a well defined classical limit) that behaves diffusively is not evident. Furthermore, based on an argument put forward in [81] (see also [31]), the far tail of $\mathcal{P}\left(|\psi|^{2}\right)$ is due to rare realizations of the disorder potential, and therefore requires an exponentially large number of eigenfunctions, which can only be accounted by disorder averaging. Here instead we study the statistical properties of eigenfunctions in a dynamical model without introducing any ensemble averaging. Our main conclusion is that in a generic dynamical system with classical diffusion, $\mathcal{P}\left(|\psi|^{2}\right)$ is described quite well by the nonlinear $\sigma$-model (NLSM). We point out that between the various theoretical works there is a considerable disagreement about the parameters that control the shape of $\mathcal{P}\left(|\psi|^{2}\right)$ and their dependence on time-reversal symmetry (TRS). More specifically, the NLSM suggests that the tail of $\mathcal{P}\left(|\psi|^{2}\right)$ in two dimensions $(2 d)$ is sensitive to TRS [23,76,77,80,81], while a direct optimal fluctuation (DOF) method predicts a symmetry independent result [82]. Recent numerical calculations [84] on the Anderson model seem to support the latter theory. This controversy, was an additional motivation for the present study.

\subsubsection{One-dimensional and two-dimensional kicked rotor models}

The one-dimensional kicked rotor (KR) model is one of the most studied and representative models in quantum chaos (for review see [41]). The classical model is described by the 
time-dependent Hamiltonian:

$$
\begin{aligned}
H & =H_{0}+V \sum_{m} \delta(t-m T), \\
H_{0}(\mathcal{L}) & =\frac{\tau}{2}(\mathcal{L}+\gamma)^{2} \\
V(\theta) & =k\left(\cos (\theta) \cos (\alpha)+\frac{1}{2} \sin (2 \theta) \sin (\alpha)\right)
\end{aligned}
$$

where $\mathcal{L}$ denotes the angular momentum and $\theta$ the conjugate angle of the rotor. The kick period is $T, k$ is the kicking strength, while $\tau$ is a constant inversely proportional to the moment of inertia of the rotor. The parameter $\alpha$ breaks time reversal symmetry (TRS) $[41,95,96]$, the parameter $\gamma$ is an irrational number whose meaning will be explained below. The Hamiltonian (3.1) describes a system which is kicked periodically in time.

One can construct a two-dimensional generalization of (3.1) taking two rotors with free Hamiltonian given by the sum of free Hamiltonians of each rotor:

$$
\begin{aligned}
H & =H_{0}+V \sum_{m} \delta(t-m T), \\
H_{0}\left(\left\{\mathcal{L}_{i}\right\}\right) & =\sum_{i=1}^{d} \frac{\tau_{i}}{2}\left(\mathcal{L}_{i}+\gamma_{i}\right)^{2},
\end{aligned}
$$

interacting with each other by the potential

$$
V\left(\left\{\theta_{i}\right\}\right)=k\left(\cos \left(\theta_{1}\right) \cos \left(\theta_{2}\right) \cos (\alpha)+\frac{1}{2} \sin \left(2 \theta_{1}\right) \cos \left(2 \theta_{2}\right) \sin (\alpha)\right)
$$

Index $i=1(2)$ is related to the first (second) rotor correspondingly. Another representation of the Hamiltonian (3.2) may be given by one rotor moving on a two-dimensional sphere. The classical equations of motion which correspond to Hamiltonians (3.1) and (3.2) can be integrated over the kick period $T$ giving a set of simple maps:

$$
\begin{aligned}
\theta_{1}(n+1)= & \theta_{1}(n)+\tau_{1} T \mathcal{L}_{1}(n) \bmod 2 \pi \\
\theta_{2}(n+1)= & \theta_{2}(n)+\tau_{2} T \mathcal{L}_{2}(n) \bmod 2 \pi \\
\mathcal{L}_{1}(n+1)= & \mathcal{L}_{1}(n)-k\left(-\sin \left(\theta_{1}(n+1)\right) \cos \left(\theta_{2}(n+1)\right) \cos (\alpha)+\right. \\
& \left.+\cos \left(2 \theta_{1}(n+1)\right) \cos \left(2 \theta_{2}(n+1)\right) \sin (\alpha)\right) \\
\mathcal{L}_{2}(n+1)= & \mathcal{L}_{2}(n)-k\left(-\cos \left(\theta_{1}(n+1)\right) \sin \left(\theta_{2}(n+1)\right) \cos (\alpha)-\right. \\
& \left.-\sin \left(2 \theta_{1}(n+1)\right) \sin \left(2 \theta_{2}(n+1)\right) \sin (\alpha)\right)
\end{aligned}
$$

where $\theta_{i}(n)$ and $\mathcal{L}_{i}(n)$ are the values of the dynamical variables taken just after the $n$-th kick. (For the one-dimensional case one should omit all terms with $i=2$ ). The motion generated by this set of maps is integrable in the absence of the kicking potential. For sufficiently small but non-zero $k$ the phase space of this system contains both regular 

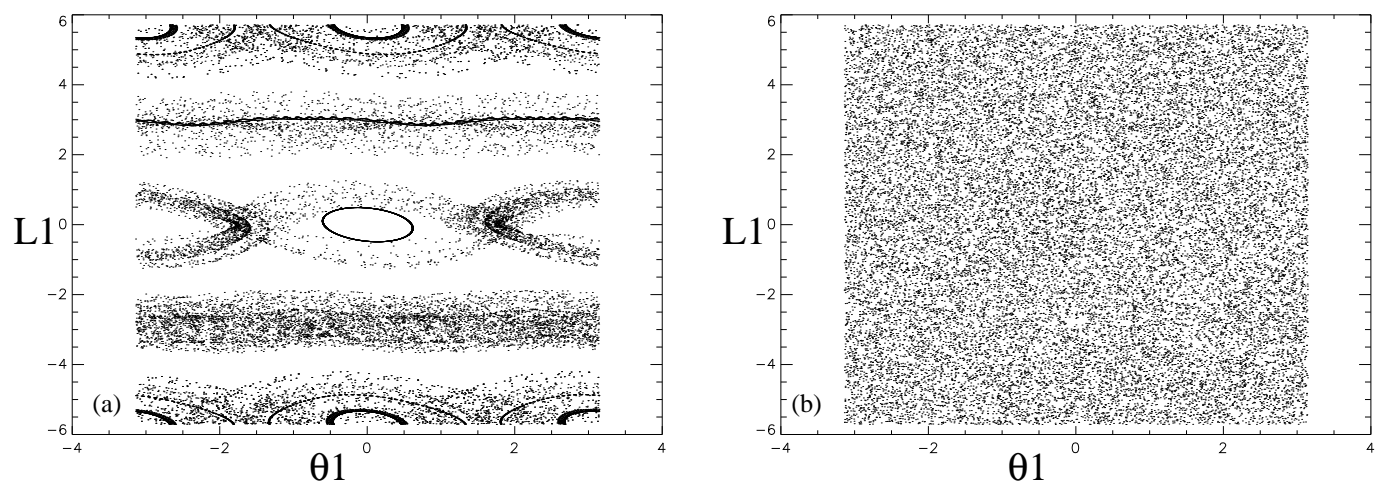

Figure 3.1: Poincaré section of the classical phase space for Hamiltonian (3.2) for (a) $k=0.36$, (b) $k=6.37$

islands and chaotic sea. When $k$ is large enough then the dynamics becomes fully chaotic (see Fig. 3.1) and there is diffusion in momentum space (Fig. 3.2) with diffusion coefficient

$$
D \equiv \lim _{t \rightarrow \infty}<\mathcal{L}^{2}(t)>/ t \simeq k^{2} / 2 T
$$

The last expression is correct within the random phase approximation [41,97] (see Appendix D.2).

If the $\mathcal{L}_{i}$ are taken $\bmod \left(2 \pi m_{i} / T \tau_{i}\right)$ where $m_{i}$ are integers, Eq. (3.1) defines a dynamical system on a torus. The quantum mechanics of this system is described by a finite-dimensional time evolution operator for one period

$$
U=\exp \left[-i H_{0}\left(\left\{\mathcal{L}_{i}\right\}\right) T / 2\right] \exp \left[-i V\left(\left\{\theta_{i}\right\}\right)\right] \exp \left[-i H_{0}\left(\left\{\mathcal{L}_{i}\right\}\right) T / 2\right]
$$

where we put $\hbar=1$. Upon quantization, additional symmetries associated with the discreteness of the momentum show up, which can be destroyed by introducing irrational values for the parameters $\gamma_{i}$ 's. The most striking consequence of quantization is the suppression of classical diffusion in momentum space due to quantum dynamical localization $[41,79]$. We introduce the eigenstate components $\boldsymbol{\Psi}_{k}(n)$ of the Floquet operator in the momentum representation by

$$
\sum_{m} U_{m n} \Psi_{k}(n)=e^{i \omega_{k} T} \boldsymbol{\Psi}_{k}(n)
$$

The quantities $\omega_{k}$ are known as quasi-energies, and their density is $\rho=T / 2 \pi$. The corresponding mean quasi-energy spacing is $\Delta=1 /\left(\rho L^{d}\right)$, where $L$ is the linear size of the system. The Heisenberg time is $t_{H}=2 \pi / \Delta$ while $t_{D}=L^{2} / D$ is the diffusion time (Thouless time). Now one can formally define a dimensionless conductance as $g=t_{H} / t_{D}=D_{k} L^{d-2}$ where $D_{k}=T D$ is the diffusion coefficient measured in number of kicks. Four length scales are important here: the wavelength $\lambda$, the mean free path $l_{M}$, the linear extent of 


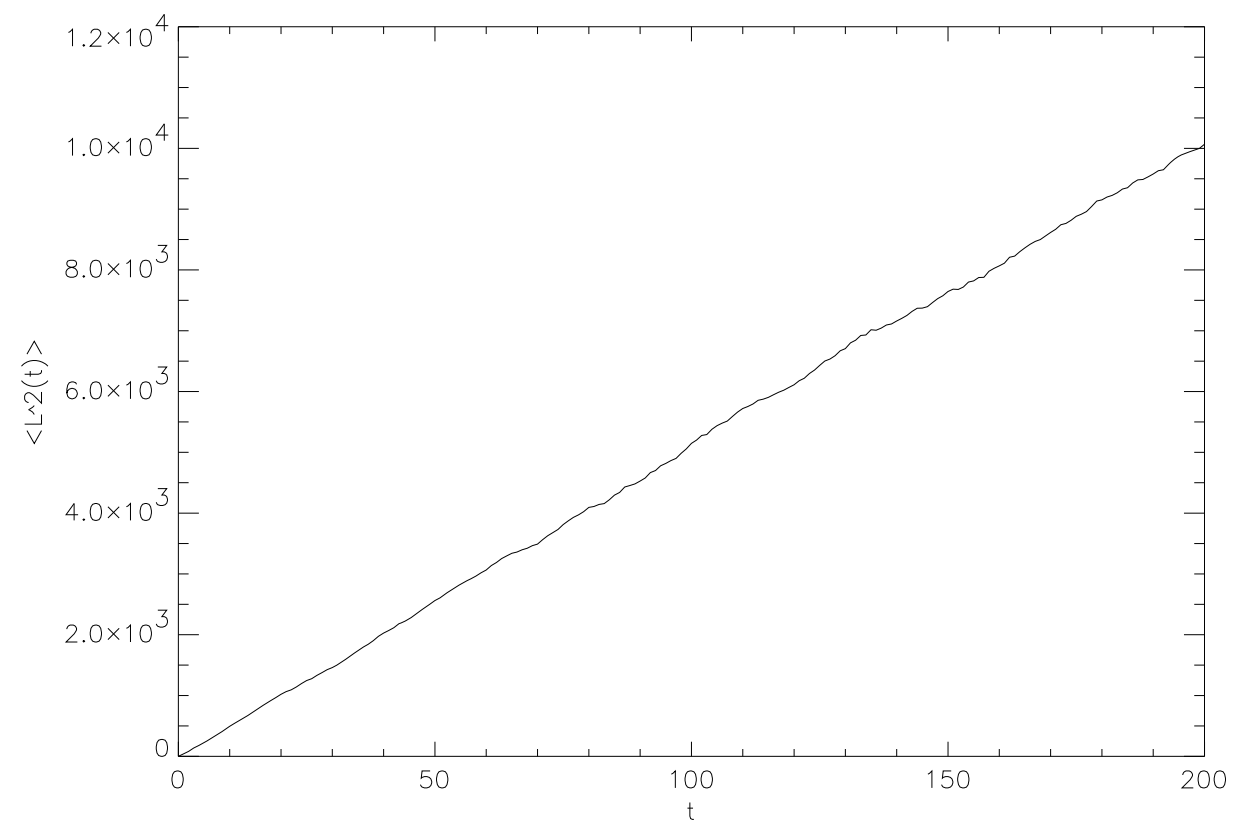

Figure 3.2: Diffusion in momentum space for $k=9.1$

the system $L$, and the localization length $\xi$. According to Refs. [23,73-77,80-82] the field theoretical predictions are derived under the conditions

$$
\lambda \ll l_{M} \ll L \ll \xi
$$

The first condition ensures that transport between scattering events may be treated semiclassically. This limit can be achieved for our system (3.1) when $k \rightarrow \infty, T \rightarrow 0$ while the classical parameter $K=k T$ remains constant. When $l_{M} \ll L$ as long as the motion is not localized (i.e. $L \ll \xi$ ) it is diffusive, since a particle scatters many times before it can traverse the system. The resulting mean free path for our system (3.1) is $l_{M} \simeq \sqrt{D}_{k}$ while the localization length for $d=1$ is $\xi \simeq D_{k} / 2$ [41] and for $d=2$ is $\xi \simeq l_{M} e^{D_{k} / 2}[38,97$ ].

Here we calculate the distribution function $\mathcal{P}\left(t=L^{d}\left|\Psi_{k}(n)\right|^{2}\right)$ by using a direct diagonalization of the Floquet operator (3.6). The TRS is broken entirely for $\alpha=5.749$. In order to test the issue of dynamical correlations, we randomize the phases of the kinetic term of the evolution operator (3.6) and calculate the resulting $\mathcal{P}(t)$. This model will be referred to as Random Phase KR (RPKR). Since all our eigenfunctions have the same statistical properties (in contrast to the Anderson cases where one should pick up only eigenfunctions having eigenenergies within a small energy interval [84-86]) we make use of all of them in our statistical analysis. The classical parameter $K$ is large enough in all cases to exclude the existence of any stability islands in phase space. The classical diffusion coefficient $D_{k}$ is calculated numerically by iterating the classical map obtained from (3.1). Below we present our numerical results and compare them to the predictions of Refs. [23,73-77, 80-82]. 


\subsubsection{One-dimensional kicked rotor}

It was shown in [31], that the effective field theory describing the semiclassical physics of the system is precisely the NLSM for quasi-one dimensional (1d) metallic wires. Such a mapping however, requires an averaging over an ensemble of rotors having the same classical limit. We point out again that in the calculations below we do not adopt such an averaging procedure.

The NLSM for quasi-1d systems can be solved exactly for the distribution function $\mathcal{P}_{\beta}(t)$, using a transfer matrix approach $[23,74,75,80,81]$. In the ballistic regime (where $g \rightarrow \infty$ ) RMT is applicable and one finds [23]

$$
\begin{aligned}
& \mathcal{P}_{(\beta=1)}^{R M T}(t)=\exp (-t / 2) / \sqrt{2 \pi t} \\
& \mathcal{P}_{(\beta=2)}^{R M T}(t)=\exp (-t) .
\end{aligned}
$$

Here $\beta$ denotes the corresponding Dyson ensemble: $\beta=1(2)$ for preserved (broken) TRS.

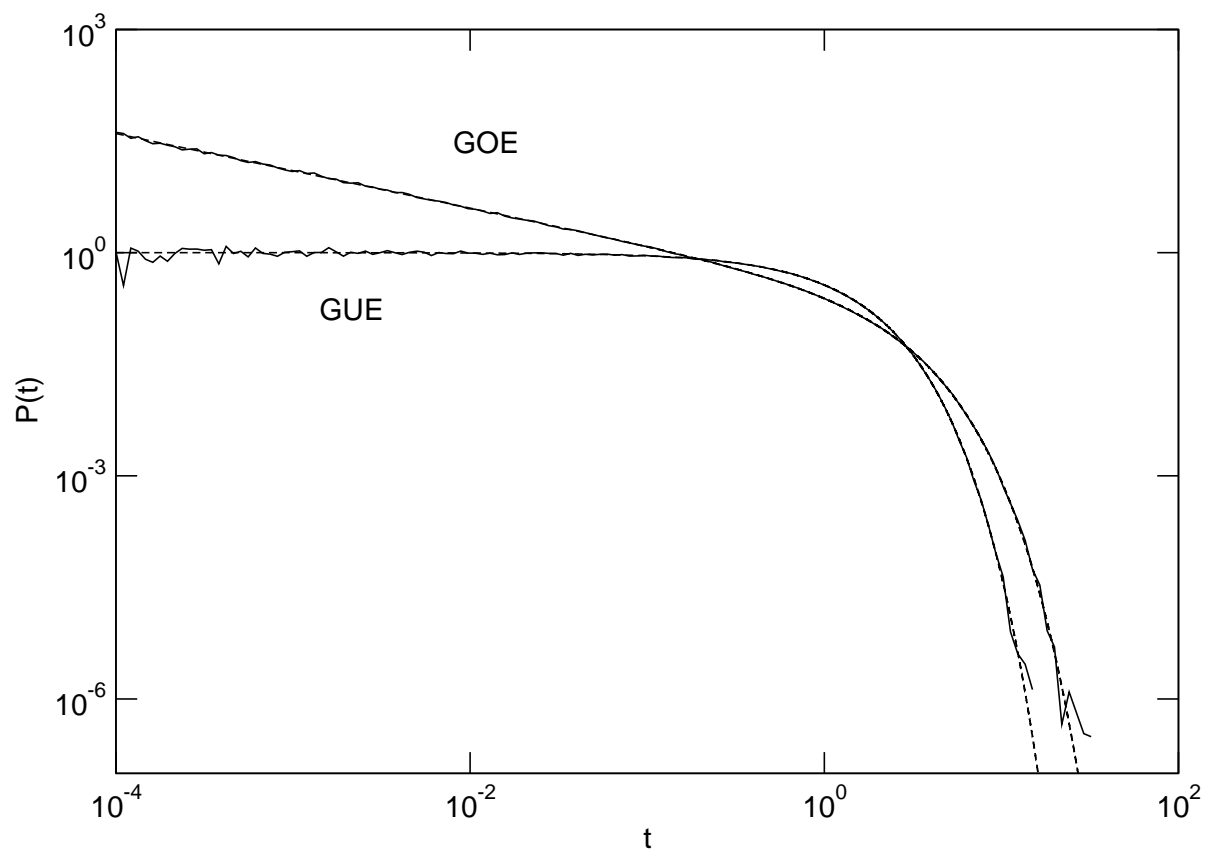

Figure 3.3: Distributions of the eigenfunction intensities in the ballistic regime for onedimensional kicked rotor (solid lines) compared with the RMT predictions (dashed lines). 
This result can be easily understood. Indeed, within the random matrix theory one assumes that all the eigenvector components are independent (the normalization of the eigenvector is not essential in the thermodynamic limit, i.e. when the number of its components becomes very large) random variables obeying Gaussian distribution. Going to the distribution of the modulus square of the components one immediately recovers Eq. (3.9). For the case of the broken time reversal symmetry one should take into account that each component has statistically independent real and imaginary parts, leading to the distribution given by Eq. (3.10). The numerical data presented in Fig. (3.3) shows that the distributions of the eigenfunction intensities in the ballistic regime for one-dimensional kicked rotor are described very nice by the RMT prediction.

As localization increases, the deviations from the RMT results of the body and the tails of the distribution $\mathcal{P}_{\beta}(t)$ become noticeable and can be parameterized by a single parameter which is the dimensionless conductance $g=D_{k} / L$.

For $t<\sqrt{D_{k} / L}$, according to all studies [74-77,80] $\mathcal{P}(t)$ is just the RMT result with polynomial corrections in powers of $L / D_{k}$, i.e. $\mathcal{P}_{\beta}(t)=\mathcal{P}_{\beta}^{R M T}(t)\left[1+\delta \mathcal{P}_{\beta}(t)\right]$. The leading term of this expansion is given by

$$
\delta \mathcal{P}_{\beta}(t) \simeq \kappa\left\{\begin{array}{ll}
3 / 4-3 t / 2+t^{2} / 4 & \text { for } \beta=1 \\
1-2 t+t^{2} / 2 & \text { for } \beta=2
\end{array},\right.
$$

where $\kappa \sim 1 / g$ is the $1 d$ diffusion propagator, which is identical for $\beta=1$ and $\beta=2$ since it is a classical quantity.

In Fig. 3.4(a) and 3.4(b) we report our numerical results for $\delta \mathcal{P}_{\beta}(t)$ for two representative values of $D_{k}$. One can clearly see that the agreement with the theoretical prediction (3.11) becomes better as $D_{k}$ increases. This is due to the fact that by increasing $D_{k}$ we are approaching the semiclassical region and therefore Eqs. (3.8) are better satisfied. At the same time higher order corrections in $\delta \mathcal{P}_{\beta}(t)$ become negligible with respect to the leading term given by Eq. (3.11). The resulting $\kappa_{1}$ and $\kappa_{2}$ obtained by the best fit of our data to Eq. (3.11) are found to be equal and in agreement with the theory (see Fig. 3.4(c)). We therefore conclude, that in a generic dynamical system, the only parameter that controls the shape of the deviations $\delta \mathcal{P}_{\beta}(t)$ is the classical diffusion propagator. Moreover, our results are in excellent agreement with the recent NLSM predictions derived in the framework of diffusive disordered systems. Finally in Fig. 3.4(c) we also report the outcome of the RPKR model. The results remain essentially the same indicating that $\mathcal{P}_{\beta}(t)$ for quasi- $1 d$ systems are insensitive to dynamical correlations.

The tail of the distribution $\left(t>D_{k} / L\right)$ deviates strongly from the RMT prediction and has a stretched exponential form $[74-77,80]$

$$
\mathcal{P}_{\beta}(t) \simeq A_{\beta} \exp \left(-2 C_{\beta} \sqrt{t}\right), \quad C_{\beta}=\beta \sqrt{D_{k} / L}
$$

where $A_{\beta}$ is a symmetry dependent constant. Our numerical results agree nicely with Eq. (3.12). In Fig. 3.5(a) we present an example of $\mathcal{P}_{\beta}(t)$. By fitting our data to Eq. (3.12) 

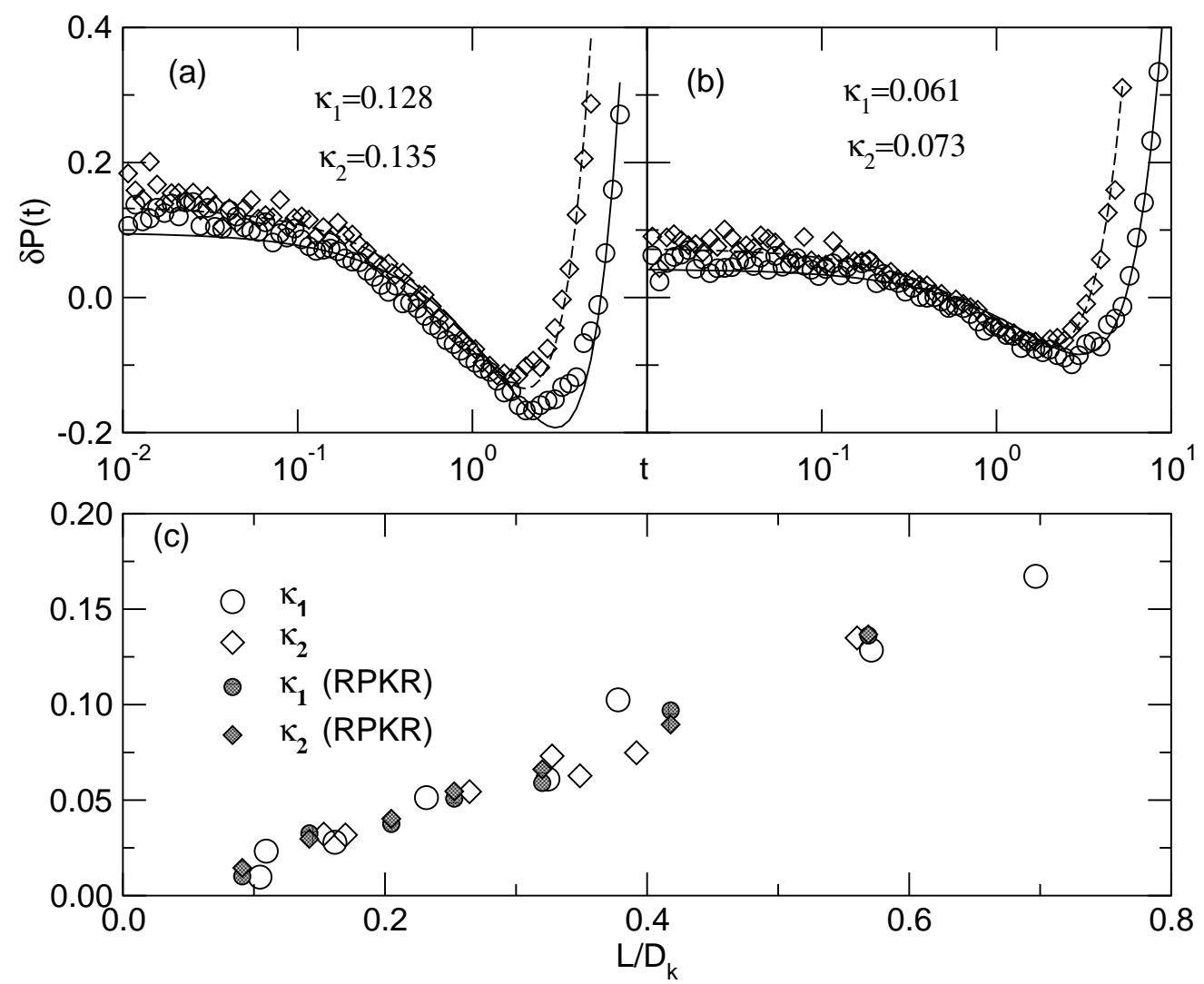

Figure 3.4: Corrections to the distribution intensities $\delta \mathcal{P}_{\beta}(t)$ for the kicked rotator model i.e. Eq. (3.1). The system size is $L=1024$, (०) $\beta=1,(\diamond) \beta=2$. The solid (dashed) lines are the best fit of (3.11) for $\beta=1(2)$ to the numerical data: (a) $D_{k} \approx 1800$ and (b) $D_{k} \approx 3150$; (c) Shows the extracted diffusion propagator $\kappa_{\beta}$ vs. $L / D_{k}$.

the coefficients $C_{1}, C_{2}$ can be extracted. In Fig. 3.5(b) we report the extracted stretched exponential coefficients $C_{\beta}$ from the best fit of (3.12) as a function of the square root of the dimensionless conductance $g=D_{k} / L$. A nice linear behavior is observed. The best linear fit $C_{\beta}=A_{\beta} \sqrt{D_{k} / L}+B_{\beta}$ yields, $A_{\beta=1}=0.41 \pm 0.05$ and $A_{\beta=2}=0.82 \pm 0.05$. The resulting ratio $R=A_{2} / A_{1}=2$ is in excellent agreement with the theoretical prediction (3.12). We have also calculated the stretched exponential coefficients $C_{\beta}$ for the RPKR model. The results for various $D_{k}$ values are summarized in Fig. 3.5(b) and show a nice agreement with the results obtained from the real Hamiltonian. 

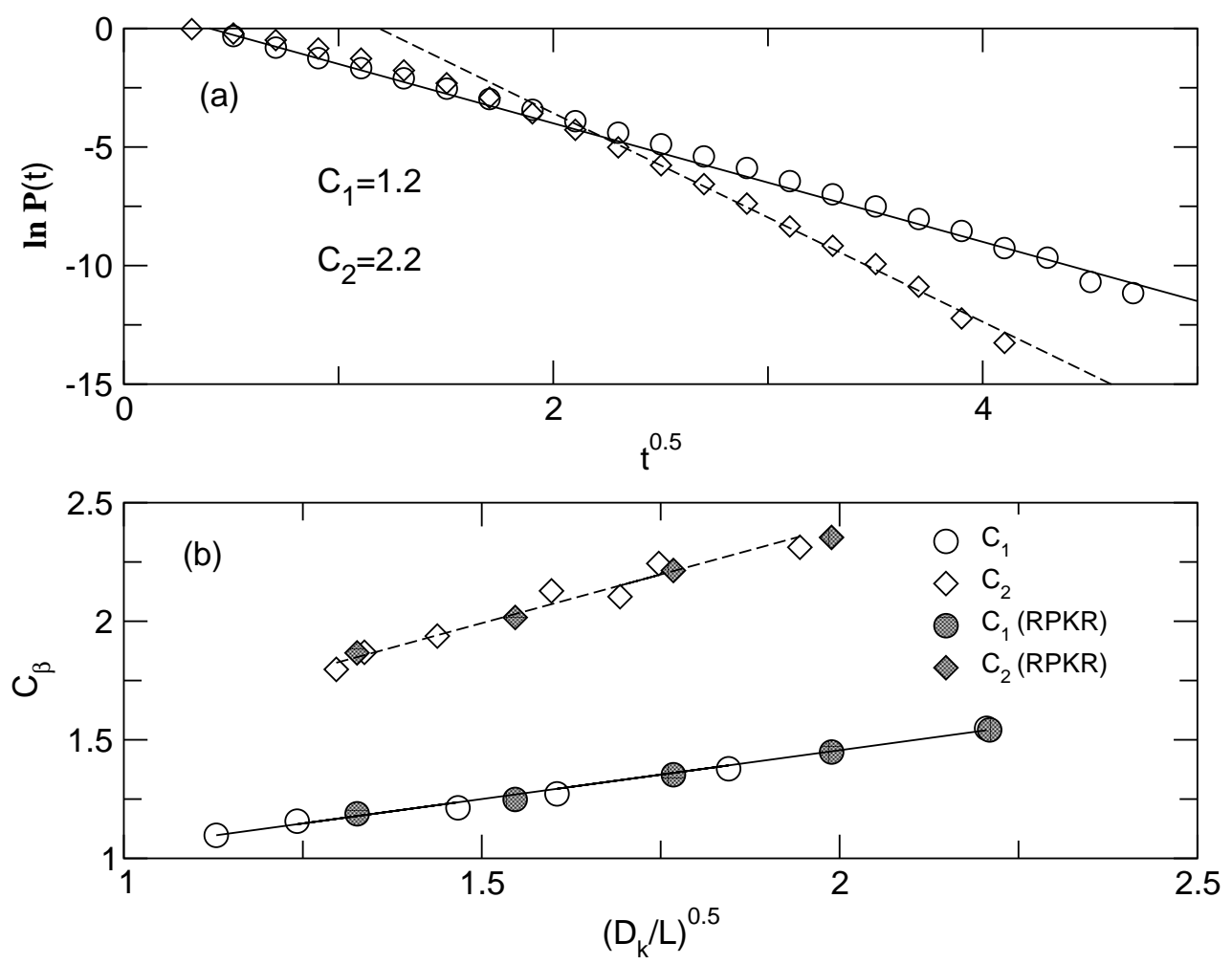

Figure 3.5: (a) Tails of the distribution $\mathcal{P}_{\beta}\left(t>D_{k} / L\right)$ for the model (3.1) with $L=1024$, $D_{k} \simeq 2625$ and for $\beta=1(\circ)$ and $\beta=2(\diamond)$. The solid (dashed) lines are the best fit of (3.12) for $\beta=1$ (2) to our data; (b) Coefficients $C_{\beta}$ vs. $\sqrt{D_{k} / L}$. The solid (dashed) lines are the best fits to $C_{\beta}=A_{\beta} \sqrt{D_{k} / L}+B_{\beta}$ for $\beta=1(2)$.

\subsubsection{Two-dimensional kicked rotor}

We start our analysis again with the ballistic regime. One expects to obtain the same statistics of the eigenfunction intensities as in the one-dimensional case, because the RMT predictions given by Eq. (3.9) and (3.10) are universal and don't depend on the dimensionality of the system. The numerical data presented in the Fig. (3.6) confirm this expectation.

According to Ref. [74], corrections to the body of $\mathcal{P}_{\beta}^{R M T}$ are still given by Eq. (3.11), but now $\kappa$ is the $2 d$ diffusion propagator.

Figures 3.7(a) and (b) show corrections to $\mathcal{P}_{\beta}^{R M T}$ for $g=D_{k} \gg 1$ for two representative values of $D_{k}$. We find again that the form of the deviations are very well described by 


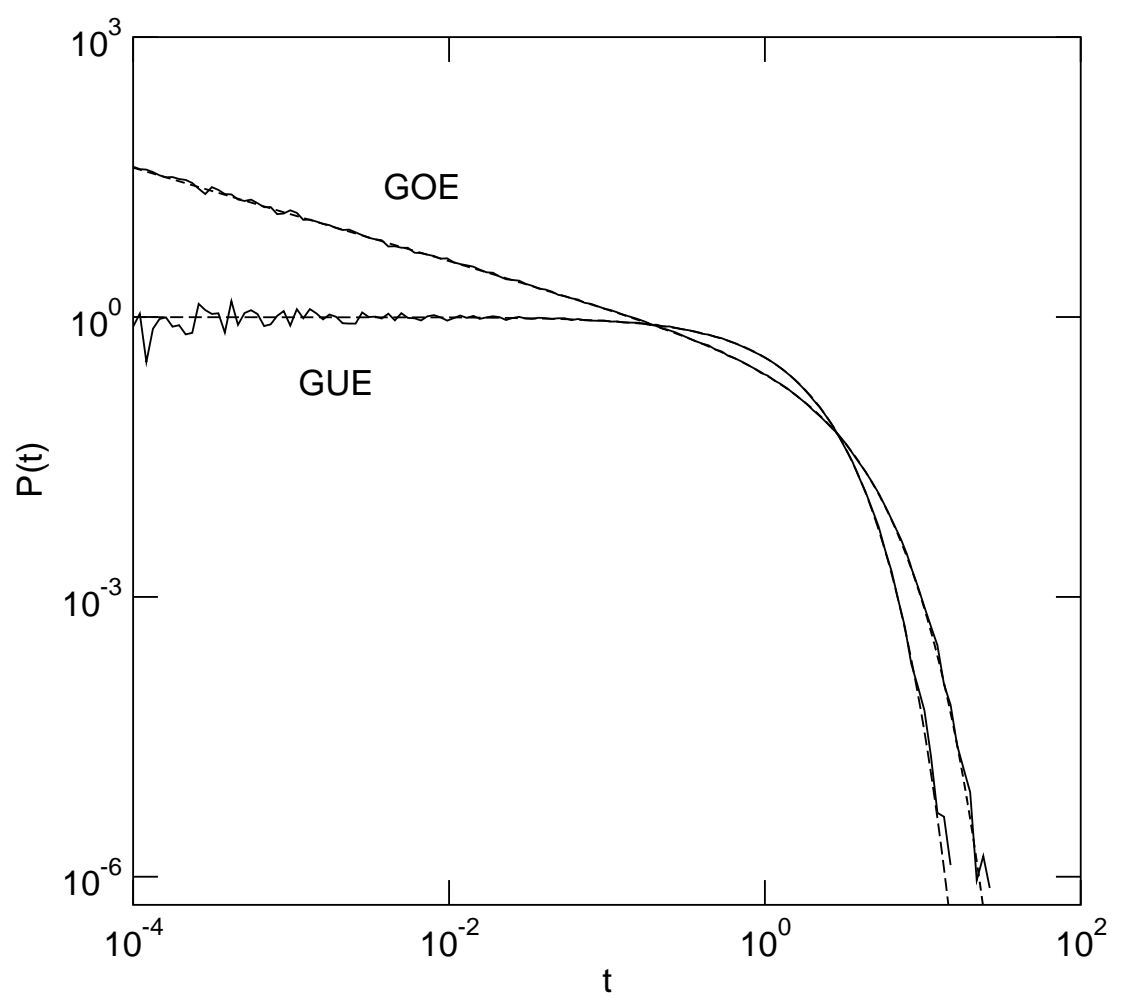

Figure 3.6: Distributions of the eigenfunction intensities in the ballistic regime for twodimensional kicked rotor (solid lines) compared with the RMT predictions (dashed lines).

Eq. (3.11) and the agreement becomes better for larger values of the diffusion constant. In Fig. 3.7(c) we summarize our results for various $D_{k}$ values. The extracted $\kappa_{\beta}$ values are obtained by the best fit of the data to Eq. (3.11). Again we find that $\kappa_{\beta}$ depends linearly on $1 / D_{k}$. However, contrary to the $1 d$-KR, here $\kappa_{1}$ and $\kappa_{2}$, are different. Moreover the best fit with $\kappa_{\beta}=A_{\beta} D_{k}^{-1}+B_{\beta}$ yields $A_{\beta=1}=5.44 \pm 0.03$ and $A_{\beta=2}=10.84 \pm 0.04$ indicating that the ratio $R=A_{2} / A_{1}$ is close to 2 , a value that could be explained on the basis of ballistic effects $[23,81,84,85]$. Taking the latter into account leads to an additional term in the classical propagator $\kappa_{\beta}=\kappa_{\text {diff }}+\frac{\beta}{2} \kappa_{\text {ball }}$. The first term is the one discussed previously and is associated with long trajectories which are of diffusive nature while the latter one is associated with short ballistic trajectories which are self-tracing [23,81]. Thus, when $\kappa_{\text {diff }} \ll \kappa_{\text {ball }}$ we get $R=2$. The calculation with the RPKR model shows, however, that the corresponding ratio is $R \simeq 1$ in agreement with the theoretical prediction for disordered systems with a pure diffusion. This indicates that dynamical correlations can be important in the $2 d$ case.

For the tails of the distributions, the result of the NLSM within a saddle-point approxi- 

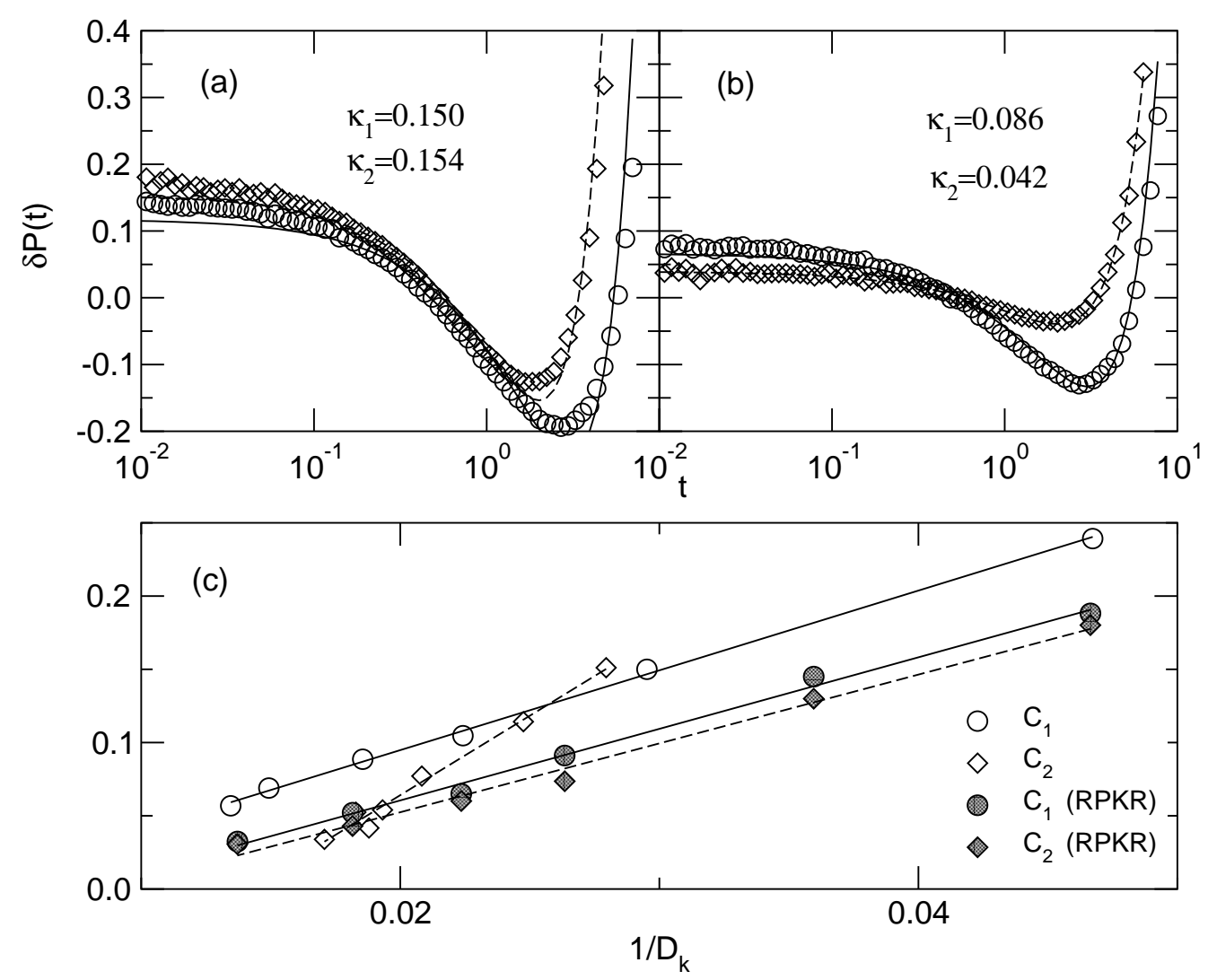

Figure 3.7: Corrections to the distribution intensities $\delta \mathcal{P}_{\beta}(t)$ for the two-dimensional kicked rotator model (3.2). The system size is $L=90,(\circ) \beta=1,(\diamond) \beta=2$. The solid (dashed) lines are the best fit of (3.11) for $\beta=1(2)$ to the numerical data: (a) $D_{k} \approx 34$ and (b) $D_{k} \approx 53$; (c) Fit parameters $\kappa_{\beta}$ vs. $D_{k}^{-1}$. The solid (dashed) lines are the best fits to $\kappa_{\beta}=A_{\beta} D_{k}^{-1}+B_{\beta}$ for $\beta=1(2)$.

mation $[23,76,77,81]$ is

$$
\mathcal{P}_{\beta}(t) \simeq \exp \left[-C_{\beta}^{\sigma}(\ln t)^{2}\right], \quad C_{\beta}^{\sigma}=\frac{\beta \pi^{2} \rho}{2} \frac{D}{\ln (L / l)} .
$$

Note that the decay in the tails of Eq. (3.13) depends on $\beta$, as in the $1 d$-KR case (see Eq. (3.12)). Recently, a DOF method was used to calculate the tails of $\mathcal{P}_{\beta}(t)$ [82]. It was found that the tails are still given by Eq. (3.13) but with a log-normal coefficient $C$ which is independent of the parameter $\beta$ :

$$
C^{\text {DOF }}=\pi^{2} \rho \frac{D}{\ln (L / \lambda)}
$$




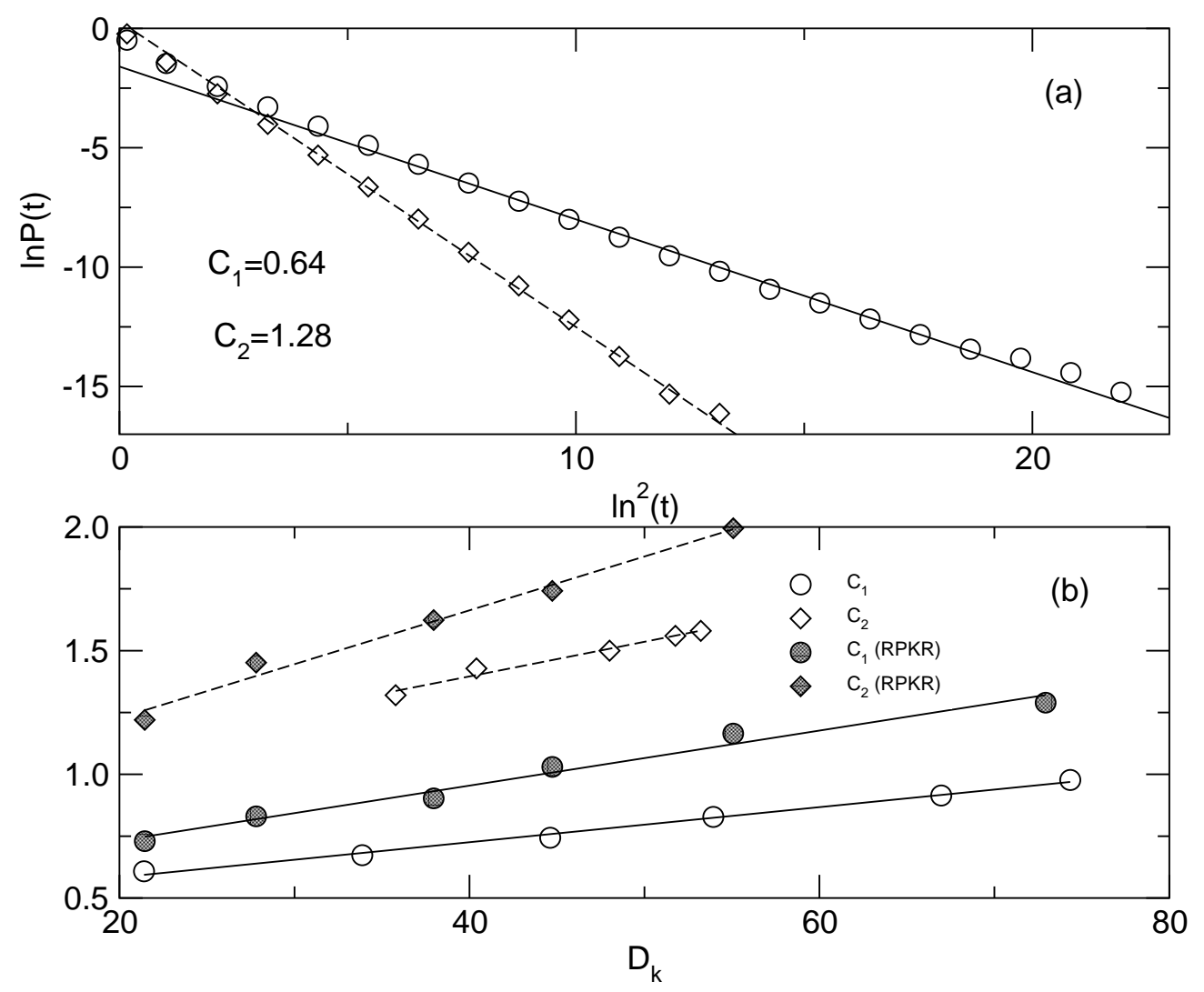

Figure 3.8: (a) Tails of the distribution $\mathcal{P}_{\beta=1}\left(t>D_{k}\right)$ for the model (3.2) and $D_{k} \simeq 35$. The system size is $L=80,(\circ) \beta=1,(\diamond) \beta=2$. The solid (dashed) lines are the best fit of (3.13) for $\beta=1(2)$ to the numerical data; (b) Fitted log-normal coefficients $C_{\beta}$ versus the classical diffusion coefficient $D_{k}$. The solid (dashed) lines are the best fits to $C_{\beta}=A_{\beta} D_{k}+B_{\beta}$ for $\beta=1(2)$.

Figure 3.8(a) shows a representative case of $\mathcal{P}_{\beta=1}\left(t>D_{k}\right)$. The tails show a log-normal behavior predicted by Eq. (3.13). In Fig. 3.8(b) we report the $\log$-normal coefficients $C_{\beta}$ extracted from the best fit to our numerical data, versus the classical diffusion coefficient. A pronounced linear behavior is observed in agreement with both theories. However one clearly sees that $C_{1}$ differs from $C_{2}$ in contrast to the DOF prediction (3.14) and to recent numerical calculations done for the $2 d$ Anderson model [84]. We point out here that in [84] the authors were not able to go to large enough values of conductance $g$ (in comparison to our study) where the theory can really be tested. In contrast, the NLSM predicts a value of 2 for the ratio $R=C_{2}^{\sigma} / C_{1}^{\sigma}$. We note that $C_{\beta}^{\sigma}$ is only the leading term in $D_{k}$. In order to calculate this ratio, we performed a fit to our data with $C_{\beta}=A_{\beta} D_{k}+B_{\beta}$. The resulting ratio was found to be $R=A_{2} / A_{1}=1.97 \pm 0.03$ in perfect agreement with 
the NLSM predictions. Finally in Fig. 3.8(b) we also present our results for the RPKR model (using the same data as the one in Fig. 3.8(c)). Again we found that the ratio $R=1.96 \pm 0.03 \approx 2$. Thus $\mathcal{P}\left(t>D_{k}\right)$ depends on TRS and is described by the NLSM. 


\subsection{Open systems: distribution of resonances and de- lay times}

For diffusive mesoscopic samples, there is no systematic investigation of $\mathcal{P}(\Gamma)$ and $\mathcal{P}(\tau)$ besides Ref. [98] where the authors have focused on the tails of $\mathcal{P}(\Gamma)$ for a quasi-1D system in the diffusive regime. In the next section we fill this gap by presenting the study of $\mathcal{P}(\Gamma)$ and $\mathcal{P}(\tau)$ for $2 D$ open systems in the diffusive regime. This study can be important for various applications like quantum dots [11], microwave cavities [29] or random lasers [12,94] where most of the theoretical treatment is limited by the RMT.

\subsubsection{Open kicked rotor model}

To proceed with the analysis of the resonance widths and delay times we turn the closed $2 D$ KR model (3.6) into an open one. To this end we impose absorption at the boundary of a square sample of size $L \times L$ in the momentum space. In other words, every time that one of the components of the two dimensional momentum $\left(\mathcal{L}_{1}, \mathcal{L}_{2}\right)$ takes on the value 1 or $L$, the particle is absorbed without coming back to the sample. Using a recently proposed recipe [99] we can write down the corresponding scattering matrix $S$ in the form (see Appendix E)

$$
\begin{aligned}
S(\omega) & =-W U e^{i \omega} \frac{1}{I-e^{i \omega} P U} W^{\dagger} \\
P & =I-W^{\dagger} W
\end{aligned}
$$

where $I$ is the $L^{2} \times L^{2}$ unit matrix and $W$ is a $M \times L^{2}$ matrix. It has only $M$ nonzero elements which are equal to one and describe at which "site" of the $L \times L$ sample we attach $M$ "leads" [in our case $M=4(L-1)$ ]. Here $W^{\dagger} W$ is a projection operator onto the boundary, while $P$ is the complementary projection operator. The scattering matrix $S_{i j}$ given by Eq. (3.15) can be interpreted in the following way: once a wave enters the sample, it undergoes multiple scattering induced by $\left[I-e^{i \omega} P U\right]^{-1}=\sum_{n=0}^{\infty}\left(e^{i \omega} P U\right)^{n}$ until it is transmitted out. It is clear therefore that the matrix $\tilde{U}=P U$ propagates the wave inside the sample. However, contrary to the closed system in which the evolution operator is unitary, the absorption breaks the unitarity of the evolution matrix $\tilde{U}$ so that all eigenvalues $\tilde{\lambda}$ move inside the unit circle. Therefore each eigenvalue can be written in the form $\tilde{\lambda}_{n}=e^{i \tilde{\omega}_{n}}=\exp \left(-i \omega_{n}-\Gamma_{n} / 2\right)$ where $\Gamma_{n}>0$ is the dimensionless resonance width of an eigenstate.

The Wigner delay time can be expressed as the sum of proper delay times $\tau_{q}$. The latter are the eigenvalues of the Wigner-Smith operator written in our case as (see Appendix E)

$$
Q(\omega) \equiv \frac{1}{i} S^{\dagger} \frac{d S}{d \omega}=-e^{-i \omega} W K^{\dagger} W^{\dagger} W K U^{\dagger} K W^{\dagger}
$$


where $K=\left(P-U^{\dagger} e^{-i \omega}\right)^{-1}$.

The corresponding classical system is described by the same set of equations (3.4) as the closed one, on top of which we add absorbing boundary conditions. Namely, we set $\mathcal{L}_{i}$ equal to zero, if $\mathcal{L}_{i}<0$ or $\mathcal{L}_{i}>L$. These conditions give to "the particles" the possibility to escape from the system. The evolution of the classical density $\rho(x, y, t)$ can be described in this case by the diffusion equation:

$$
\frac{\partial \rho}{\partial t}=\frac{D}{4} \Delta \rho
$$

with absorbing boundary condition

$$
\vec{J}_{n} \equiv-\frac{D}{4}(\vec{\nabla} \rho)_{n}=-\Phi_{n}
$$

which sets the flux at the boundary $\vec{J}_{n}$ to be equal to the number of particles $\Phi_{n}$ carried out under one iteration of the map. The solution of Eq. (3.17) can be represented as the superposition of the diffusive eigenmodes $v_{k}(x, y)$ :

$$
\rho(x, y, t)=\sum_{k=1}^{\infty} c_{k} e^{-\gamma_{k} t} v_{k}(x, y)
$$

where $\gamma_{k}$ are the corresponding eigenvalues and $c_{k}$ are coefficients determined by the initial condition. The asymptotic behavior of the density is governed by the smallest eigenvalue $\gamma_{1} \equiv \Gamma_{\mathrm{cl}}$. As a consequence one has an exponential decay of the classical survival probability $P(t)=\iint d x d y \rho(x, y, t)$ :

$$
P(t) \propto e^{-\Gamma_{c l} t},
$$

The classical decay rate $\Gamma_{c l} \sim D / L^{2}$ can be estimated as the inverse time needed for the particle to reach the boundary (Thouless time). The exact value of the classical decay rate can be obtained as the solution of the corresponding eigenvalue problem or from the numerical calculation of $P(t)$ (see Fig. 3.9).

Below we present our theoretical considerations and compare them with the numerical data obtained for the $2 D \mathrm{KR}$ model. The parameters of the model were chosen in such a way that the conditions (3.8) discussed in the previous section were fulfilled. In order to improve our statistics, we randomized the phases of the kinetic term of the evolution operator (3.6) and used a number of different realizations. In all cases we had at least 60000 data for statistical processing.

\subsubsection{Resonance widths distribution}

In this section we demonstrate that the distribution of resonance widths is determined by the diffusive classical dynamics of the corresponding closed system and depends on the 


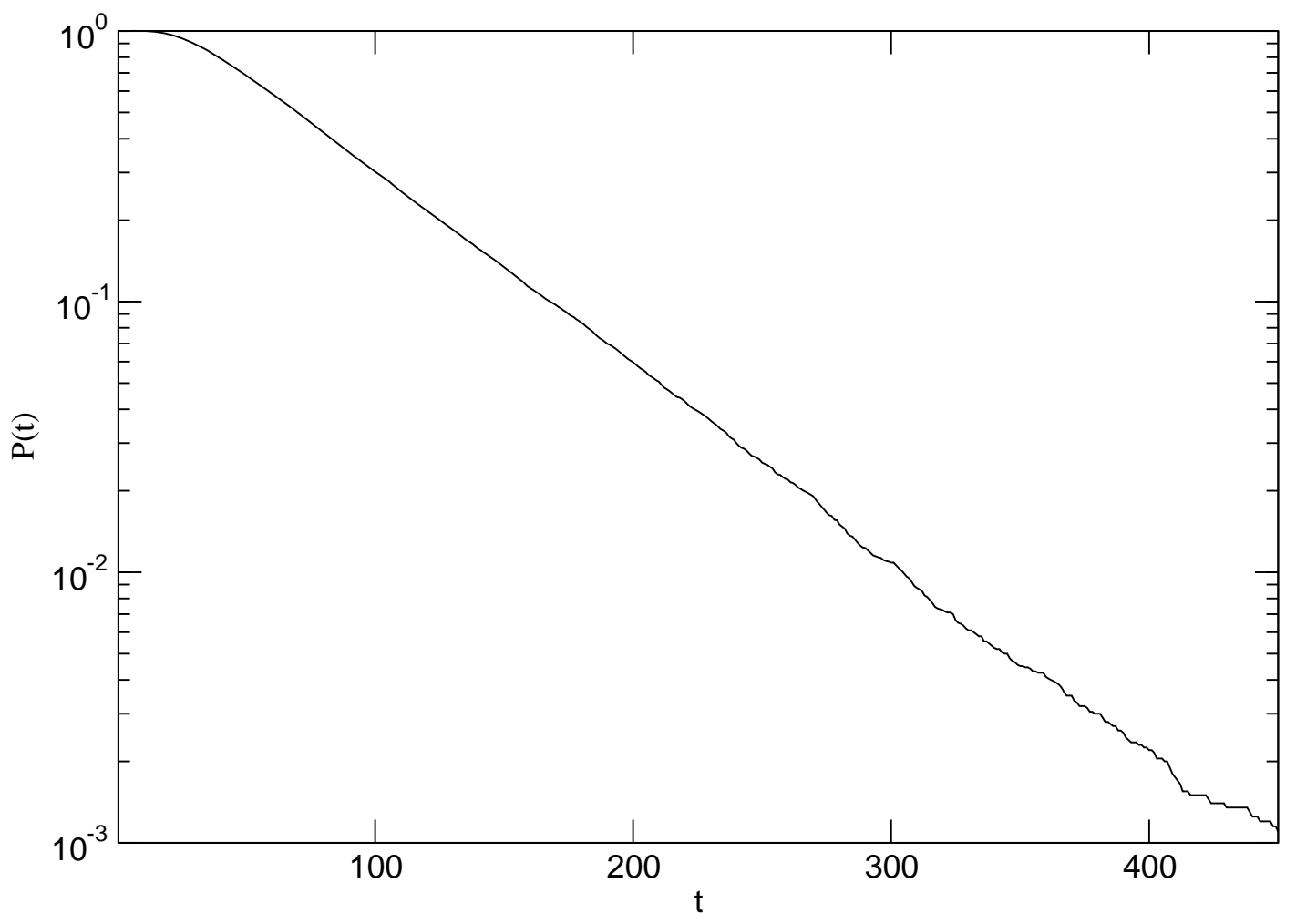

Figure 3.9: Exponential decay of the classical survival probability for open kicked rotor model.

time-reversal symmetry. Specifically, the resonance width distribution $\mathcal{P}(\Gamma)$ is given by

$$
\begin{aligned}
\mathcal{P}\left(\Gamma<\Gamma_{c l}\right) & \sim \exp \left(-C_{\beta}(\ln \Gamma)^{2}\right), \quad \text { where } \quad C_{\beta} \sim \beta D \\
\mathcal{P}\left(\Gamma \gtrsim \Gamma_{c l}\right) & \sim \sqrt{\frac{D}{L^{2}}} \frac{1}{\Gamma^{3 / 2}}
\end{aligned}
$$

where $\beta$ denotes the symmetry class.

We start our analysis with the study of resonance width distribution $\mathcal{P}(\Gamma)$ for $\Gamma<\Gamma_{c l}$. The small resonances $\Gamma<\Delta$ can be associated, with the existence of prelocalized states of the closed system which were discussed in the previous section. They consist of a short-scale bump (where most of the norm is concentrated) and they decay rapidly in a power law fashion from the center of localization [23,82]. One then expects that states of this type with localization centers at the bulk of the sample are affected very weakly by the opening of the system at the boundaries. In first order perturbation theory, considering the opening 
as a small perturbation we obtain

$$
\frac{\Gamma}{2}=\left\langle\Psi\left|W^{\dagger} W\right| \Psi\right\rangle=\sum_{n \in \text { boundary }}|\Psi(n)|^{2} \sim L|\Psi(L)|^{2}
$$

where $|\Psi(L)|^{2}$ is the wavefunction intensity of a prelocalized state at the boundary. At the same time the distribution of $\theta=1 / \sqrt{L} \Psi(L)$ for large values of the argument is found to be of log-normal type [82]:

$$
\mathcal{P}(\theta) \sim \exp \left(-\pi^{2} D \ln ^{2}\left(\theta^{2}\right)\right)
$$

Using this together with Eq. (3.22) we obtain

$$
\mathcal{P}(1 / \Gamma) \sim \exp \left(-\pi^{2} D \ln ^{2}(1 / \Gamma)\right)
$$

We would like to stress that the expression for $\mathcal{P}(\theta)$, must be corrected by including the TRS factor $\beta$ in the exponent. This is due to the fact that the Optimal Fluctuation Method, which was used to derive the above expression for $\mathcal{P}(\theta)$, does not describe the effect of breaking TRS in a correct way [42,100]. Taking all the above into account we end up with the expression given in Eq. (3.21).

The numerical data reported in Fig. 3.10 support the validity of the above considerations. However, we would like to mention that the perturbative argument is valid only for the case of very small resonances i.e. $\Gamma<\Delta$, whereas our numerical data indicate that one can extend the log-normal behavior of $\mathcal{P}(\Gamma)$ up to resonances with $\Delta<\Gamma<\Gamma_{c l}$.

Next we turn to the analysis of $\mathcal{P}(\Gamma)$ for $\Gamma \gtrsim \Gamma_{c l}$. In Fig. 3.11(a) we report our numerical results for $\mathcal{P}(\Gamma)$ with preserved (broken) TRS for two representative values of $D$. An inverse power law $\mathcal{P}(\Gamma) \sim \Gamma^{-1.5}$ is evident in accordance with Eq. (3.21). (The behavior of the extreme large $\Gamma$ tails of $\mathcal{P}(\Gamma)$ is essentially determined by the coupling to the leads which is model dependent. Their relative number is proportional to $M / L^{2} \sim L^{-1}$ and therefore they are statistically insignificant.) From the figure it is clear that this part of the distribution is independent of the symmetry class, in contrast to the small resonance distribution discussed above.

The following argument provides some understanding of the behavior of $\mathcal{P}(\Gamma)$ for $\Gamma \gtrsim \Gamma_{c l}$. First we need to recall that the inverse of $\Gamma$ represents the quantum lifetime of a particle in the corresponding resonant state escaping into the leads. Moreover we assume that the particles are uniformly distributed inside the sample and diffuse until they reach the boundaries, where they are absorbed. Then we can associate the corresponding lifetimes with the time $t_{R} \sim 1 / \Gamma_{R} \sim R^{2} / D$ a particle needs to reach the boundaries, when starting a distance $R$ away. This classical picture can be justified for all states with $\Gamma \gtrsim \Gamma_{c l} \sim D / L^{2}$. The relative number of states that require a time $t<t_{R}$ in order to reach the boundaries (or equivalently the number of states with $\Gamma>\Gamma_{R}$ ) is

$$
\mathcal{P}_{\text {int }}\left(\Gamma_{R}\right)=\int_{\Gamma_{R}}^{\infty} \mathcal{P}(\Gamma) d \Gamma \sim \frac{S\left(t_{R}\right)}{L^{2}}
$$



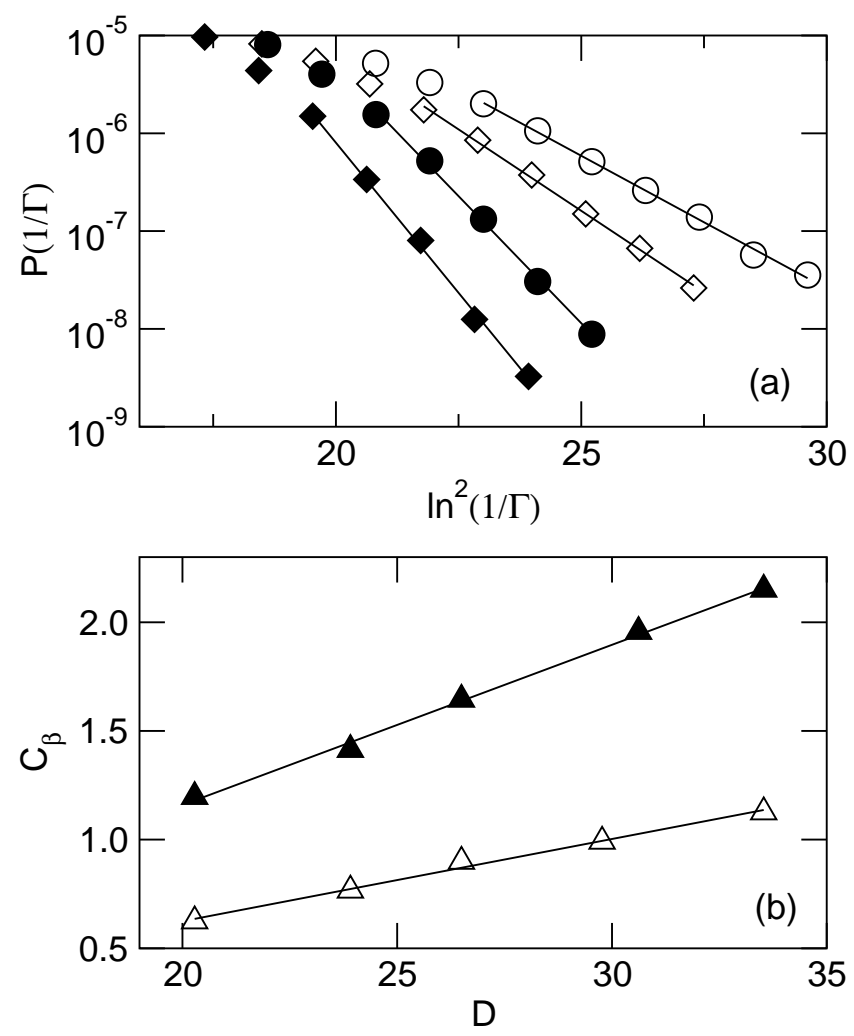

Figure 3.10: (a) The distribution of resonance widths (plotted as $\mathcal{P}(1 / \Gamma)$ vs. $1 / \Gamma$ ) for $\Gamma<\Gamma_{c l}$ for two representative values of $D$. The system size in all cases is $L=80$. Filled symbols correspond to broken TRS. The solid lines are the best fit of Eq. (3.21) for $\beta=1(2)$ to the numerical data. (b) Coefficients $C_{\beta}$ vs. $D$. The solid lines are the best fits to $C_{\beta}=A_{\beta} D+B_{\beta}$ for $\beta=1(2)$. The ratio $R=A_{2} / A_{1}=1.95 \pm 0.03$

where $S\left(t_{R}\right)$ is the area populated by all particles with lifetimes $t<t_{R}$. In the case of open boundaries we get

$$
\mathcal{P}_{\mathrm{int}}\left(\Gamma_{R}\right) \sim \frac{L^{2}-(L-2 R)^{2}}{L^{2}} \sim \sqrt{\frac{\Gamma_{c l}}{\Gamma_{R}}}-\frac{\Gamma_{c l}}{\Gamma_{R}}
$$

For $\Gamma_{R}>\Gamma_{c l}$ the first term in the above equation is the dominant one and thus Eq. (3.21) follows.

Here it is interesting to point that a different way of opening the system might lead to a different power law behavior for $\mathcal{P}(\Gamma)$. Such a situation can be realized if instead of opening the system at the boundaries we introduce "one-site" absorber (or one "lead") 

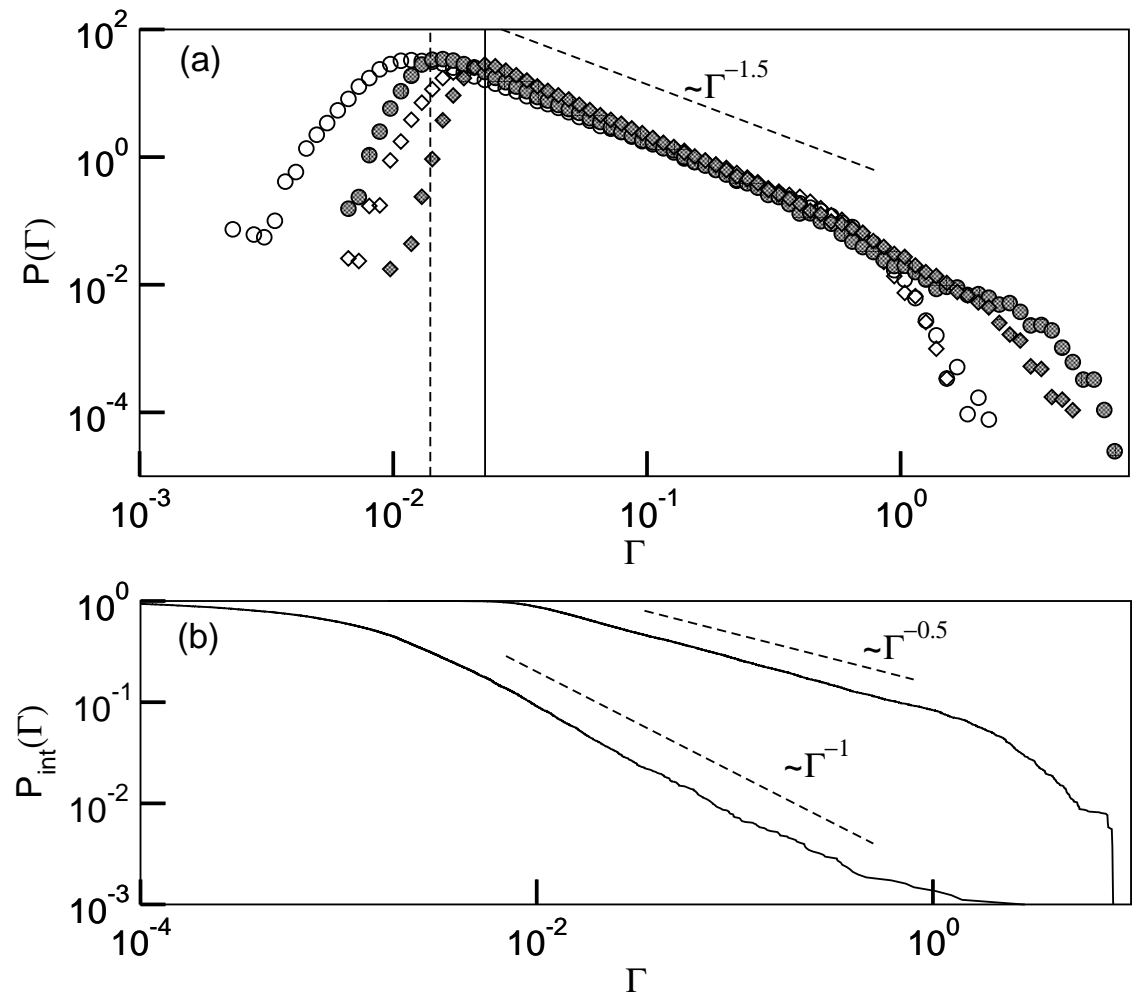

Figure 3.11: (a) The resonance width distribution $\mathcal{P}(\Gamma)$ for preserved TRS and $D=20.3$ (०) and $D=33.5(\diamond)$. The corresponding filled symbols represent $\mathcal{P}(\Gamma)$ for broken TRS and the same values of $D$. The dashed (solid) vertical line mark the classical decay rate $\Gamma_{c l}$ for $D=20.3(D=33.5)$. (b) The $\mathcal{P}_{\text {int }}(\Gamma)$ for a sample with nine leads (lower curve). For comparison we plot also the $\mathcal{P}_{\text {int }}(\Gamma)$ for the same sample but when we open the system from the boundaries. The dashed lines correspond to the theoretical predictions (3.26) and (3.27).

somewhere in the sample. In such a case we have

$$
\mathcal{P}_{\text {int }}\left(\Gamma_{R}\right) \sim \frac{S\left(t_{R}\right)}{L^{2}}=\frac{R^{2}}{L^{2}}=\frac{D t_{R}}{L^{2}} \sim \frac{\Gamma_{c l}}{\Gamma_{R}} .
$$

The above result is valid for any number $M$ of "leads" such that the ratio $M / L^{2}$ scales as $1 / L^{2}$. In Fig. 3.11(b) we report the integrated resonance width distribution $\mathcal{P}_{\text {int }}(\Gamma)$ for the case with nine "leads" attached somewhere to the $2 D$ sample.

A straightforward generalization of our arguments for 3D systems in the diffusive regime 
gives

$$
\mathcal{P}_{\text {int }}\left(\Gamma_{R}\right) \sim \sqrt{\frac{\Gamma_{c l}}{\Gamma_{R}}}-2 \frac{\Gamma_{c l}}{\Gamma_{R}}+\frac{4}{3}\left(\frac{\Gamma_{c l}}{\Gamma_{R}}\right)^{\frac{3}{2}}
$$

which for $\Gamma_{R}>\Gamma_{c l}$ leads to the same universal expression as in Eq. (3.21). Similarly, the analog of Eq. (3.27) in 3D is

$$
\mathcal{P}_{\text {int }}\left(\Gamma_{R}\right) \sim\left(\frac{\Gamma_{c l}}{\Gamma_{R}}\right)^{\frac{3}{2}}
$$

It is interesting to compare the above prediction (3.21) with the results of the random matrix theory. In the general case, Fyodorov and Sommers [8] proved that the distribution of scaled resonance widths $\gamma=\Gamma / \Delta$ for the unitary random matrix ensemble, is given by

$$
\mathcal{P}(\gamma)=\frac{(-1)^{M}}{\Gamma(M)} \gamma^{M-1} \frac{d^{M}}{d \gamma^{M}}\left(\mathrm{e}^{-\gamma \pi q} \frac{\sinh (\gamma \pi)}{(\gamma \pi)}\right)
$$

where $M$ is the number of open channels and the parameter $q$ controls the degree of coupling with the channels. In the limit of $M \gg 1$, which is relevant for the comparison with Eq. (3.21), Eq. (3.30) reduces to the following expression [8]

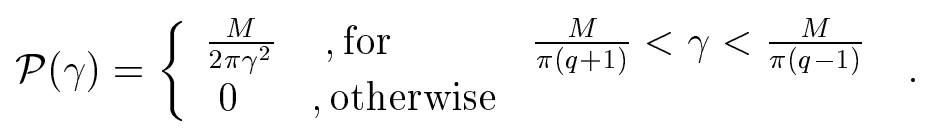

One can see two essential distinctions between this result and Eq. (3.21). Firstly, the power law $P(\Gamma) \sim 1 / \Gamma^{2}$ is not the same as the power law predicted by Eq. (3.21) for large resonances $P(\Gamma) \sim 1 / \Gamma^{3 / 2}$. Since this difference appears in the "classical" part of the distribution, it can be explained as a difference in the classical dynamics of a particle inside the system: ballistic (RMT) versus diffusive motion. Indeed, taking into account that for ballistic system $R \sim v t$ and $\Gamma_{c l} \sim v / L$, where $v$ is the velocity of the particle, one immediately finds from Eq. (3.26) that $\mathcal{P}_{\text {int }}\left(\Gamma_{R}\right) \sim \Gamma_{c l} / \Gamma_{R}$ for $\Gamma_{R}>\Gamma_{c l}$, in agreement with the RMT prediction $P(\Gamma) \sim 1 / \Gamma^{2}$. Secondly, according to Eq. (3.31) there is a gap in the distribution of the resonance widths: there are no resonances with widths smaller than $\frac{M}{\pi(g+1)}$. The existence of the gap can be understood, if one relates the small resonances to the coupling of the wavefunctions to the leads. Since the wavefunctions in the RMT are extended, the probability to find a wavefunction, which is weakly coupled to all $M$ channels, goes to zero when the number of channels becomes very large $M \gg 1$. In the diffusive regime, in contrast, there are prelocalized states, which are weakly coupled to the leads. Due to their existence the distribution of the small resonance widths has a non-trivial behavior described by Eq. (3.21).

\subsubsection{Wigner delay times distribution}

The main result of this section is the distribution of the Wigner delay times, which is given by the following expressions: 


$$
\begin{aligned}
& \mathcal{P}\left(\tau \lesssim \Gamma_{c l}^{-1}\right) \sim \frac{1}{\tau^{3 / 2}} \exp (-\sigma / \tau) \\
& \mathcal{P}\left(\tau>\Gamma_{c l}^{-1}\right) \sim \exp \left(-C_{\beta}(\ln \tau)^{2}\right)
\end{aligned}
$$

where $\sigma$ is some constant of order unity and $C_{\beta} \sim \beta D$ is the same constant as in Eq.(3.21). Our theoretical understanding will be based on the following relation (see Eq. (A.26))

$$
\tau(\omega)=\sum_{n=1}^{L^{2}} \frac{\Gamma_{n}}{\left(\omega-\omega_{n}\right)^{2}+\Gamma_{n}^{2} / 4}
$$

which connects the Wigner delay time and the poles of the $S$-matrix. Let us start with the far tails. It is evident that large times $\tau(\omega) \sim \Gamma_{n}^{-1}$ correspond to the cases when $\omega \simeq \omega_{n}$ and $\Gamma_{n} \ll 1$. Then for the distribution of delay times we obtain

$$
\mathcal{P}(\tau) \sim \int d \Gamma \mathcal{P}(\Gamma) \delta(\tau-1 / \Gamma)
$$

Then the small resonance width asymptotic given by Eq. (3.21) yields the log-normal law of Eq. (3.32).

Now we estimate the behavior of $\mathcal{P}(\tau)$ for $\tau \lesssim \Gamma_{c l}^{-1}$. In this regime many short-living resonances contribute to the sum (3.33). We may therefore consider $\tau$ as a sum of many independent positive random variables each of the type $\tau_{n}=\Gamma_{n} x_{n}$, where $x_{n}=\delta \omega_{n}^{-2}$. Assuming further that $\delta \omega_{n}$ are uniformly distributed random numbers we find that the distribution $\mathcal{P}\left(x_{n}\right)$ has the asymptotic power law behavior $1 / x_{n}^{3 / 2}$. As a next step we find that the distribution $\mathcal{P}\left(\tau_{n}\right)$ decays asymptotically as $1 / \tau_{n}^{3 / 2}$ where we use that $\mathcal{P}\left(\Gamma_{n}\right) \sim$ $1 / \Gamma_{n}^{3 / 2}$. Then the corresponding $\mathcal{P}(\tau)$ is known to be a stable asymmetric Levy distribution $L_{\mu, 1}(\tau)$ of index $\mu=1 / 2$ [101] which has the form given in Eq. (3.32) at the origin. We point out here that the asymptotic behavior $\mathcal{P}(\tau) \sim 1 / \tau^{3 / 2}$ emerges also for chaotic/ ballistic systems where the assumption of uniformly distributed $\delta \omega_{n}$ is the only crucial ingredient (see for example [8]).

Since $\tau=\sum_{i=1}^{M} \tau_{q}$, we expect the behavior of the distribution of proper delay times $\mathcal{P}\left(\tau_{q}\right)$ to be similar to $\mathcal{P}(\tau)$ for large values of the arguments (for $\tau \gg 1$ we have $\tau \sim \tau_{q}^{\max }$ ). Moreover, from the numerical point of view $\mathcal{P}\left(\tau_{q}\right)$ can be studied in a better way because a larger set of data can be generated easily. Our numerical findings for $\mathcal{P}\left(\tau_{q}\right)$ are reported in Fig. 3.12 and are in nice agreement with Eq. (3.32), even for moderate values of $\tau_{q}$. We stress here that the dashed lines in Fig. 3.12, have slopes equal to $C_{\beta}$ taken from the corresponding log-normal tails of $\mathcal{P}(\Gamma)$.

Finally we would like to compare our result (3.32) with the results known from the random matrix theory. Although the distributions of the proper delay times $[102,103]$ and partial delay times (defined as a derivative of the partial phase $\theta_{i}$ of the $S$-matrix with respect to 


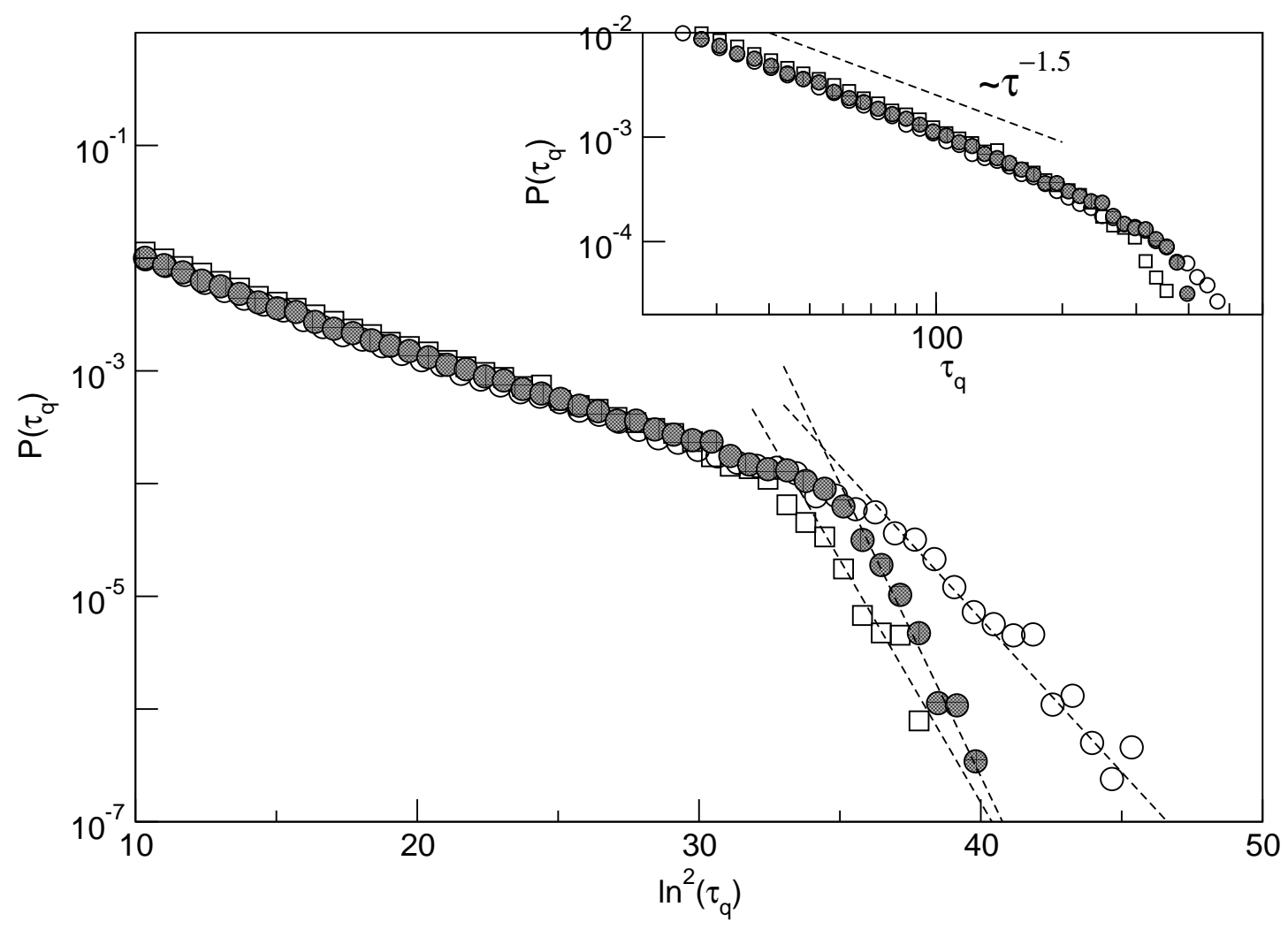

Figure 3.12: The proper delay times distribution $\mathcal{P}\left(\tau_{q}\right)$ for $D=20.3(\circ)$ and $D=29.8(\square)$. The $(\bullet)$ correspond to $D=20.3$ but now with broken TRS. The dashed lines have slopes equal to $C_{\beta}$ extracted from the corresponding $\mathcal{P}(\Gamma)$ (see Fig. 1b). In the inset we report $\mathcal{P}\left(\tau_{q}\right)$ for moderate values of $\tau_{q}$ in a double logarithmic scale.

energy) [8] have been calculated recently, there is no analytical expression for the distribution of the Wigner delay times. Nevertheless using consideration similar to one presented above (see the discussion for $\tau \lesssim \Gamma_{c l}^{-1}$ ) one can argue [8] that the part of $\mathcal{P}(\tau)$ for $\tau \lesssim \Gamma_{c l}^{-1}$ is the same in the RMT as stated in Eq. (3.32). However, the distribution of the large delay times in the RMT is expected to have a power law behavior $\mathcal{P}(\tau) \sim 1 / \tau^{2+\beta M / 2}$ with $M$ being the number of open channels. This is in contrast with a log-normal tail stated in Eq. (3.32). 


\subsection{Outlook}

This chapter is devoted to the investigation of chaotic and disordered systems characterized by the classical diffusion. The first part deals with study of the closed system. Specifically, we perform a detailed numerical analysis of the eigenfunction intensities $\mathcal{P}(t)$ of the standard kicked rotor on a torus and its two-dimensional generalization. Based on these results, we concluded that the distribution $\mathcal{P}(t)$ of generic quantum dynamical systems with diffusive classical limit is affected by the existence of prelocalized states. An example of one of the prelocalized states is presented in Fig. 3.13. The deviations from RMT are well described by field theoretical methods developed for disordered systems. In particular, in a clarifying way we have resolved the controversy between DOF and NLSM by demonstrating that the dependence of the tails of $\mathcal{P}_{\beta}(t)$ on TRS is described correctly by the latter theoretical approach.

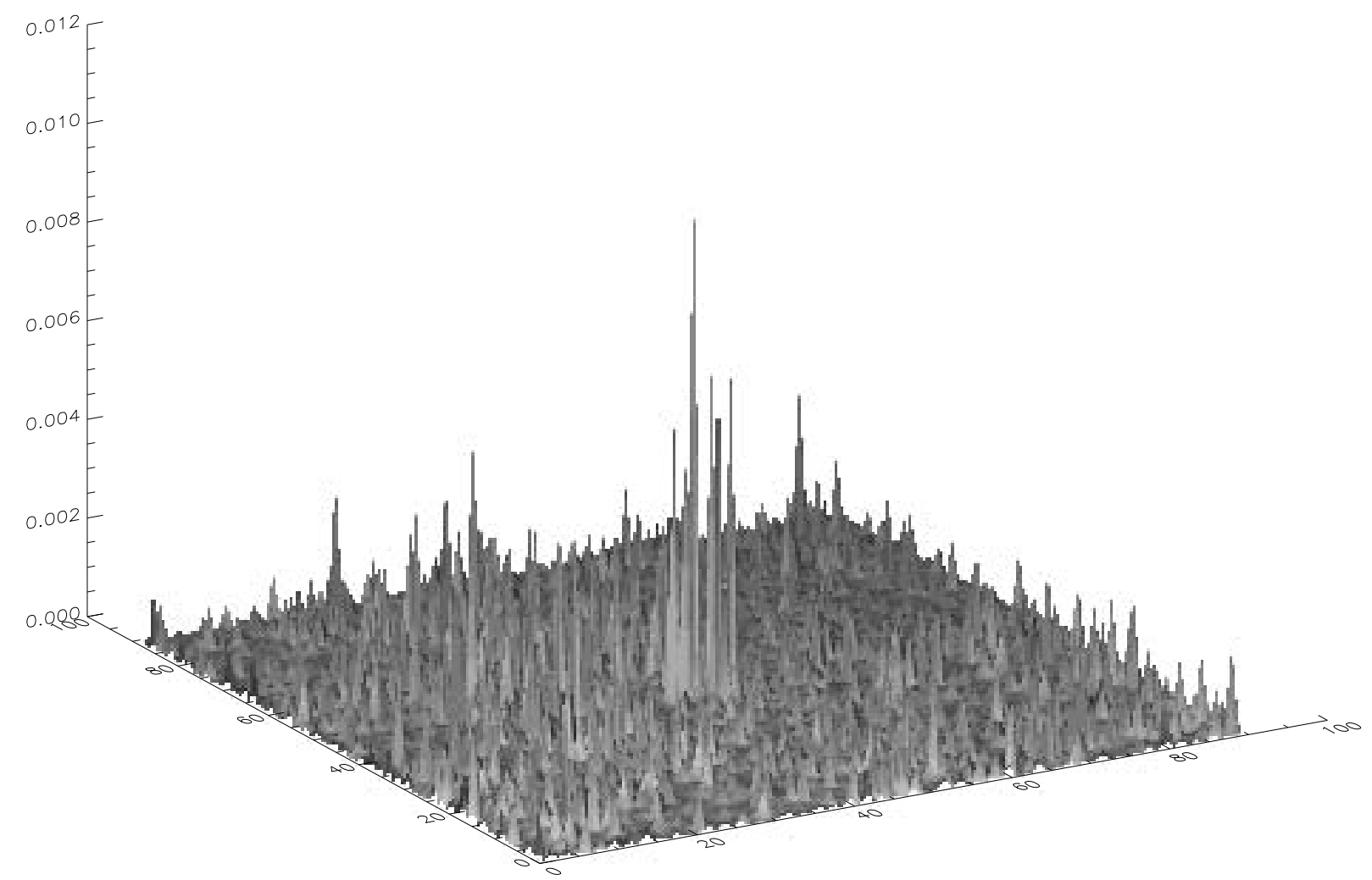

Figure 3.13: An example of anomalously localized state. The size of the system is $L \times L=90 \times 90$, the diffusion coefficient $D=33.8$ 
The study of the eigenfunction intensities can be extended to the open systems. Here one is interesting in the statistical properties of the non-orthogonal eigenvectors of the effective non-Hermitian Hamiltonians. The knowledge of their statistical properties is significant in understanding the dynamical properties of scattering and specifically the relaxation of the survival probability (see Eq. (1.11). Another important application appear in the frame of quantum optics, where it was shown that the quantum limited linewidth of a laser depends on the non-orthogonality of the cavity modes (Petermann factor) [104]. Up to now the results on the statistical properties of non-orthogonal eigenvectors are associated with chaotic/ballistic systems where the RMT predictions are applicable [104,105]. However, nothing is known for the diffusive regime, where deviations from the RMT are expected to appear.

The second part of this chapter deals with the study of the open system. We investigate the distribution of the resonance widths $\mathcal{P}(\Gamma)$ and Wigner delay times $\mathcal{P}(\tau)$. We obtain the forms of these distributions (log-normal for large $\tau$ and small $\Gamma$, and power law in the opposite case) for different symmetry classes and show that they are determined by the underlying diffusive classical dynamics and by the existence of the prelocalized states. Our theoretical arguments are supported by extensive numerical calculations.

Although the arguments, we used to explain the behavior of $\mathcal{P}(\Gamma)$ and $\mathcal{P}(\tau)$, can be easy generalized to the three-dimensional case, the numerical test of these predictions has not been still performed. Moreover the study of three-dimensional case would allow to investigate these distribution at the critical point of the metal-insulator transition. The first attempt to attack this problem was done in Ref. [106], but a detailed understanding is still required. 


\section{Chapter 4}

\section{Criticality: one-dimensional quasiperiodic systems}

This chapter is concerned with one-dimensional quasi-periodic systems at critical conditions. Many interesting results are known for the closed systems of this type. In particular, the energy spectra of these systems have fractal structure. Not much is known about the corresponding open systems. For this reason we study here the statistical properties of resonances, Wigner delay times, survival probability and scattering matrix autocorrelation function for two different types of quasi-periodic tight-binding models. We demonstrate how the behavior of these quantities is related to the fractal dimension of the spectrum. The first model we study is the Harper model, which shows a metal-insulator transition in one dimension. The second one is the Fibonacci chain model, which allows us to test our predictions in a more general way. 


\subsection{Quasiperiodic models}

\subsubsection{Harper model}

For the Bloch electron in magnetic field Peierls [107] and Onsager [108] suggested the following effective-Hamiltonian concept. One starts with the energy dispersion $\epsilon_{n}(\vec{k})$ for the $n$-th band without magnetic field. Then one substitutes the wave vector $\vec{k}$ by the operator

$$
k \rightarrow \frac{1}{\hbar}(\hat{p}-e \hat{A}),
$$

where $\hat{A}$ is the vector potential. The new operator is considered as an effective one-band Hamiltonian associated with the $n$-th band and gives in the classical limit the semiclassical equation of motion. The justification of this procedure was discussed in the literature by many authors (see for example [109,110]). Using the effective Hamiltonian one can write the stationary Schrödinger equation for the $n$-th band as

$$
\epsilon\left(\frac{1}{\hbar}(\hat{p}-e \hat{A})\right) \psi=E \psi
$$

where $\psi(x, y)$ is the wavefunction at the position $(x, y)$ and $E$ is the corresponding eigenenergy.

The second approximation we use is the simplest form of the energy dispersion. Since $\epsilon_{n}(\vec{k})$ is a periodic function of $\vec{k}$, its simplest non-trivial form contains only lower Fourier components:

$$
\epsilon_{n}(\vec{k})=E_{0}\left(\cos k_{x} a+\cos k_{y} a\right)
$$

where $a$ is the lattice constant and $2 E_{0}$ is the band width of the unperturbed band.

The magnetic field in $z$ direction yields the vector potential $\hat{A}=B(0, \hat{x}, 0)$ in the Landau gauge. We substitute now Eq. (4.3) into Eq. (4.2) and obtain

$$
\left[E_{0} \cos \left(\frac{a \hat{p}_{x}}{\hbar}\right)+\cos \left(\frac{a \hat{p}_{y}}{\hbar}-\frac{a e B x}{\hbar}\right)\right] \psi(x, y)=E \psi(x, y)
$$

Using that $\cos \alpha=\frac{1}{2}\left(e^{i \alpha}+e^{-i \alpha}\right)$ and taking into account that $e^{i a \hat{p}_{x} / \hbar}$ and $e^{i a \hat{p}_{x} / \hbar}$ are translations operators one has

$$
\frac{1}{2} E_{0}\left[\psi(x+a, y)+\psi(x-a, y)+e^{-i a B x / \hbar} \psi(x, y+a)+e^{i a B x / \hbar} \psi(x, y-a)\right]=E \psi(x, y)
$$

The wavefunction at the point $(x, y)$ is coupled now only to the four neighbor points. This is the result of the simplest form of the energy dispersion (4.3) we used. 
Introduction of the dimensionless variables $n=x / a, l=y / a, \tilde{E}=2 E / E_{0}$ (further we use $E$ again instead of $\tilde{E})$, and $\sigma=a^{2} e B / h$ yields

$$
\psi_{n+1, l}+\psi_{n-1, l}+e^{-i 2 \pi n \sigma} \psi_{n, l+1}+e^{i 2 \pi n \sigma} \psi_{n, l-1}=E \psi_{n, l}
$$

Since the coefficients of this equation don't depend on $l$, one can use the free propagating waves as the ansatz in $y$-direction:

$$
\psi_{n, l}=e^{i \nu l} \psi_{n}
$$

Substituting this equation into Eq. (4.6) we find the one-dimensional tight-binding equation

$$
\psi_{n+1}+\psi_{n-1}+\lambda \cos (2 \pi n \sigma-\nu) \psi_{n}=E \psi_{n},
$$

where $\lambda=2$. This equation was studied first by Harper [111] and is referred to as the Harper equation.

The parameter $\sigma$ in this equation plays a very important role. It is proportional to the magnetic field and gives the number of flux quanta in a unit cell. It is also equal to the ratio of the time $\frac{m a^{2}}{2 \pi \hbar}$ needed to pass through one unit cell (having a momentum $\frac{2 \pi}{a} \hbar$ ) to the inverse cyclotron frequency $\frac{e B}{m}$. When $\sigma$ is an irrational number the period of the effective potential $V_{n}=\lambda \cos (2 \pi \sigma n)$ is incommensurate with the lattice period. In the following we consider generic irrationals which cannot be approximated "too well" by rationals. To this end we take $\sigma$ as the limit of successive rationals $p / q$, so that the potential becomes

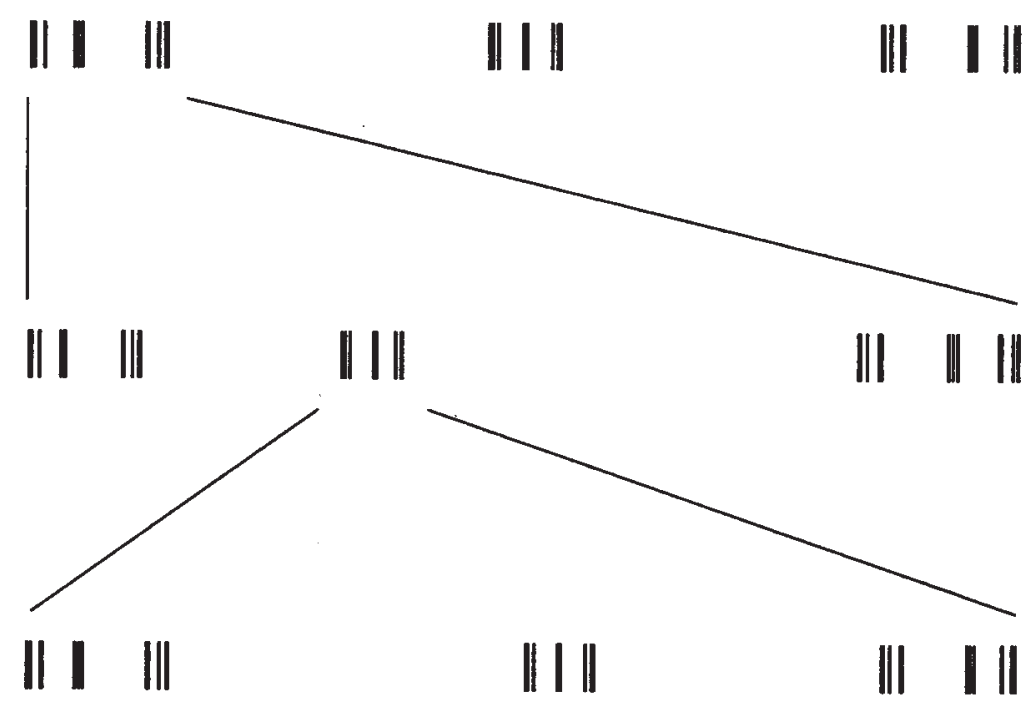

Figure 4.1: The spectrum of the Harper model by $\lambda=2$ and $\sigma$ taken as a rational approximant of the golden mean. The magnifications of the parts of the spectrum show its self similar structure. (The figure is taken from Ref. [112]) 
periodic on the lattice with a period $q$. Then we can define a scaling procedure where the incommensurate limit $q \rightarrow \infty$ becomes equivalent with the thermodynamic limit. The parameter $\nu$, the wave vector of the plane wave in $y$-direction, is in contrast not so significant and therefore one can put it to be equal to zero.

\subsubsection{Quasiperiodic tight-binding Hamiltonians}

The Harper equation (4.8) belongs to the class of one-dimensional tight-binding models with a quasiperiodic potential.

$$
\psi_{n+1}+\psi_{n-1}+V_{n} \psi_{n}=E \psi_{n}
$$

where $V_{n}$ is given by some quasiperiodic sequence. This class of models describes in particular the physics of quasicrystals [113]. The quasicrystals differ from normal crystals on one hand and from amorphous matter on the other hand, by possessing a new type of long-range translational order - quasiperiodicity. Since the discovery of quasicrystals in $1984[114,115]$ they are the subject of intensive research both in physical and mathematical communities [116].

One of the basic models describing quasicrystals is the Fibonacci chain model [117-120], which was implemented experimentally using semiconductor superlattices [121]. The potential $V_{n}$ in this model takes only two values $+V$ and $-V$ arranged in a Fibonacci sequence as follows. Two basis sequences are giving just by two different letters $S_{0}=A$ and $S_{1}=B$. Then we define the sequence $S_{n}$ as the sequence $S_{n-1}$ followed by the sequence $S_{n-2}$. According to this definition we have

$$
\begin{aligned}
S_{0} & =A \\
S_{1} & =B \\
S_{2} & =B A \\
S_{3} & =B A B \\
S_{4} & =B A B B A \\
& \ldots
\end{aligned}
$$

The interest to the quasiperiodic systems is motivated not only by their unusual properties discussed in the next section, but also by their various experimental realizations. Among them are superconductor superlattices [121,122], Wigner crystals [123] and microwave experiments [124]. 


\subsubsection{Properties of the Harper and Fibonacci chain models}

It is known that the states of the closed Harper model are extended when $\lambda<2$, and the spectrum consists of bands. For $\lambda>2$ the spectrum is point-like and all states are exponentially localized. The most interesting case is the critical point $\lambda=2$ where one has a metal-insulator transition. The eigenstates at this point are critical, i.e. they possess self-similar fluctuations on all scales [46,125-127], while the spectrum is a zero measure Cantor set (see Fig. 4.1).

A Cantor set can be characterized by the so-called fractal dimension defined as follows. Consider a set $\mathcal{T}$ contained in a $n$-dimensional space. Let $N(\epsilon)$ be the minimum number of $n$-dimensional cubes of side-length $\epsilon$ needed to cover $\mathcal{T}$. Then the fractal (box-counting) dimension of $\mathcal{T}$ is defined as

$$
D_{0}=\lim _{\epsilon \rightarrow \infty} \frac{\ln N(\epsilon)}{\ln (1 / \epsilon)}
$$

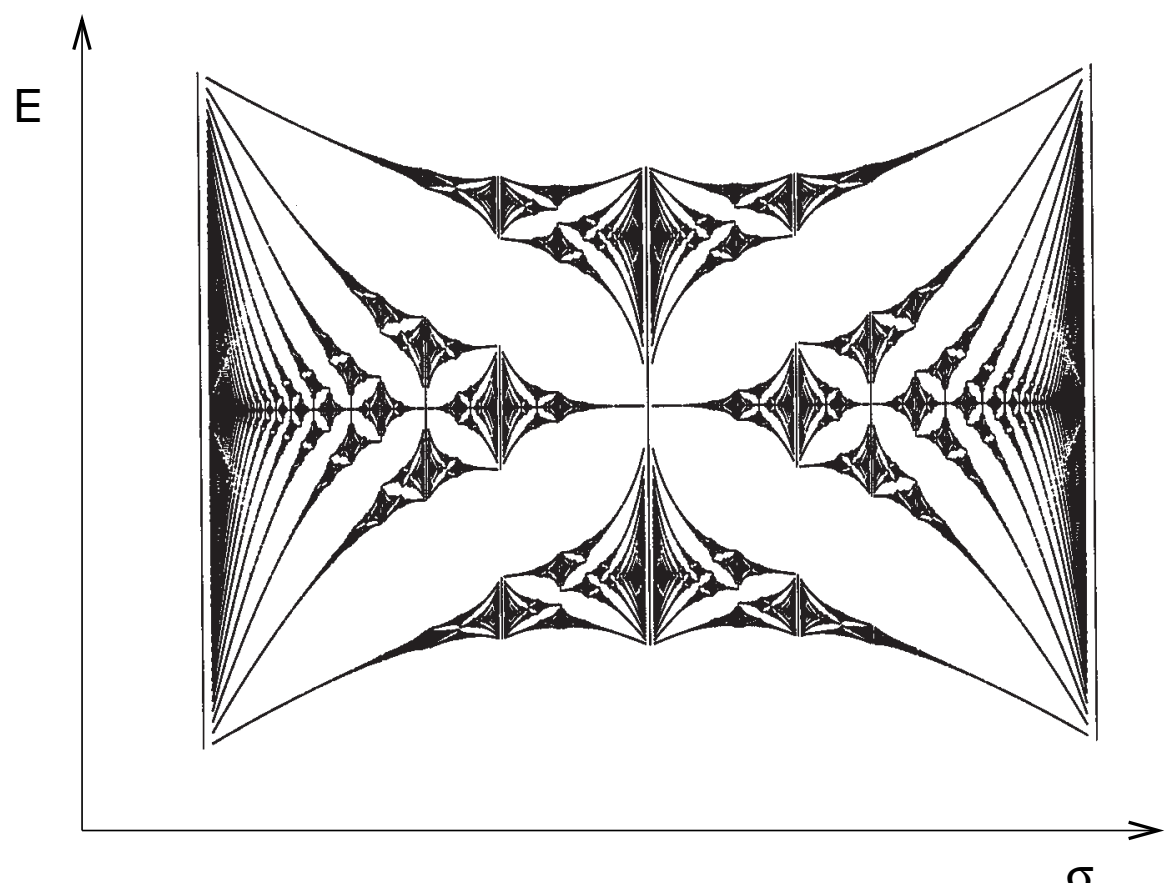

Figure 4.2: The Hofstadter butterfly shows the dependence of the spectrum of the Bloch electron from the magnetic field (from 0 to 1 flux quanta in a unit cell). (The figure is taken from Ref. [112])

The spectrum of the Harper model at the critical point has the fractal (box-counting) dimension $D_{0}^{E} \leq 0.5[128,129]$. One of the fascinated phenomenon known for the critical point of the Harper model is so-called Hofstadter butterfly [130] (see Fig. 4.2) - a selfsimilar structure appearing when one plots the spectrum of the Bloch electron as a function of the magnetic field. 
Similarly, the spectrum of the Fibonacci model is a Cantor set with zero Lebesgue measure for all $V>0$. The fractal dimension of the spectrum depends non-trivially on the strength of the on-site potential $D_{0}^{E}=D_{0}^{E}(V)$ (Fig. 4.3).

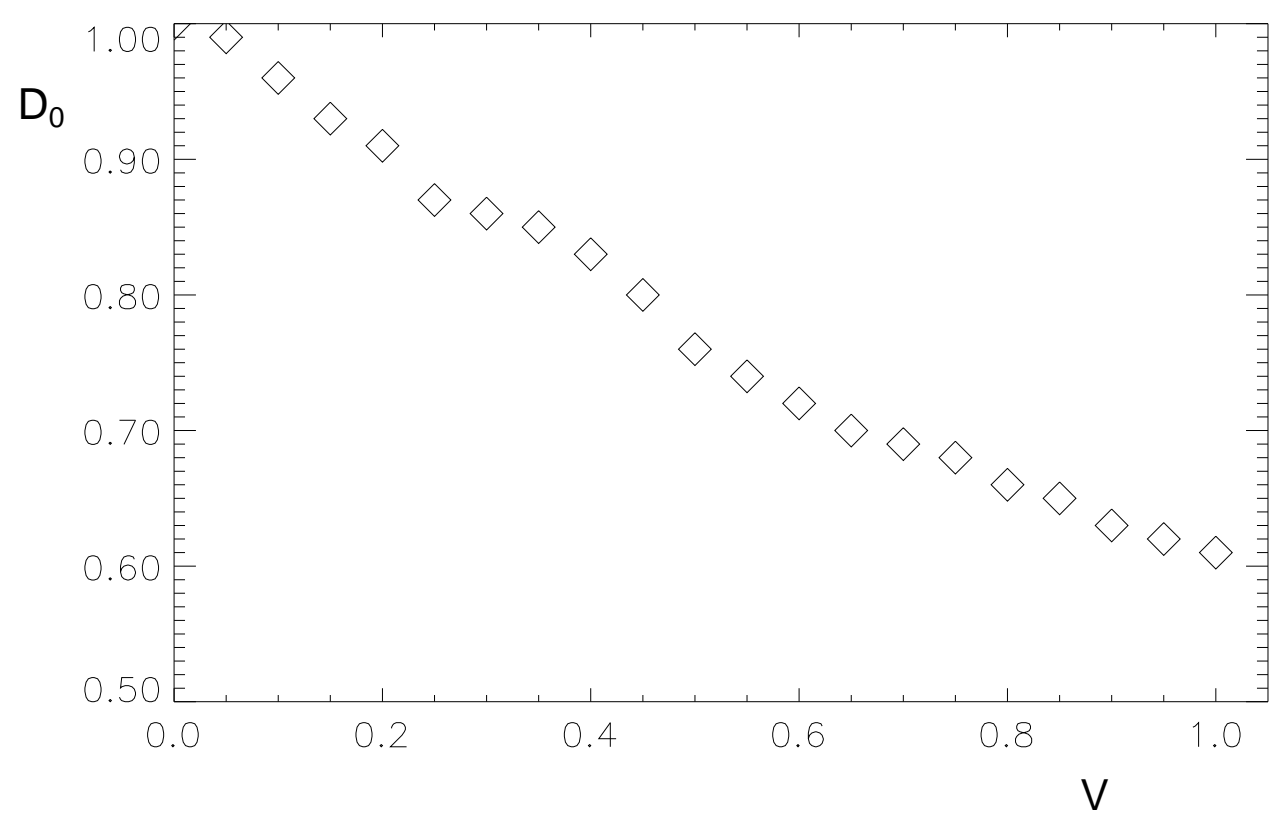

Figure 4.3: The box-counting dimension $D_{0}^{E}$ of the spectrum of the Fibonacci chain model as a function of the on-site potential strength.

The fractal nature of the spectrum leads to an unusual energy level spacing distribution $P(s) \sim s^{-1-D_{0}^{E}}$ which is a signature of level clustering [46,127]. This is in strong contrast to the level repulsion predicted by the RMT $P(s) \sim s^{\beta}$. Traces of the fractality can be also seen in the dynamics. Specifically, the variance of a wave packet spreads anomalously in time as $\operatorname{var}(t) \sim t^{2 D_{0}^{E}}$ (see Fig. 4.4) [46,127,131].

The corresponding closed systems were intensively studied during the last two decades and many interesting phenomena were found. All of them appear due to the fractal nature of the spectrum. In the following we show what are the fingerprints of the fractal spectrum for open systems.

\subsection{Open system}

We consider the same scattering setup as the one used in Chapter 1. It consists of the finite quasiperiodic sample of size $L$ and one perfect lead attached on the left side (see Fig. 2.2). Thus the system is described by the tight-binding equation (4.9), where $V_{n}$ 


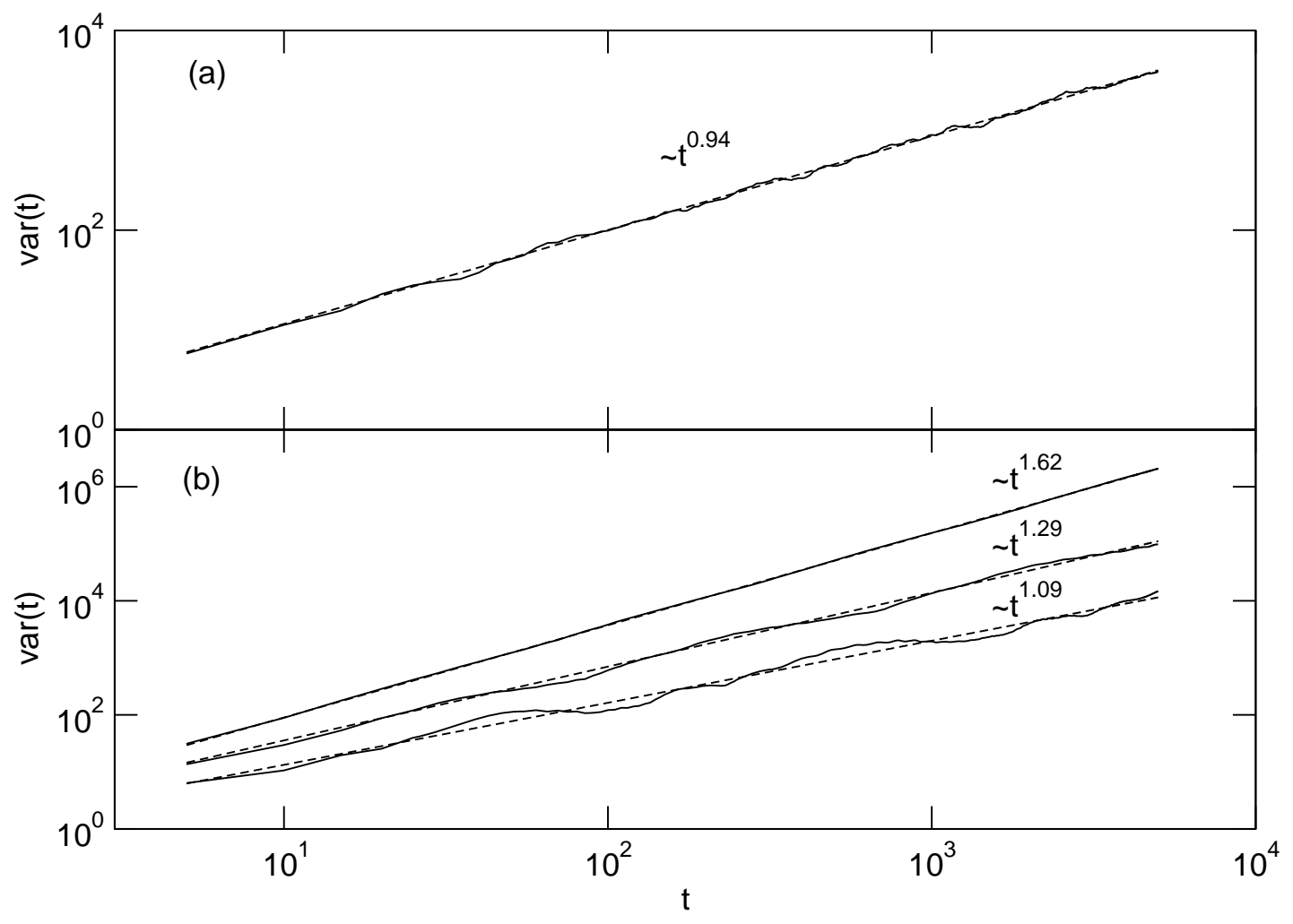

Figure 4.4: The variance of the wave packet as a function of time. The numerical calculation are plotted by solid lines, while dashed lines represent the best fit with a power low dependence, which is in reasonable agreement with the prediction $\operatorname{var}(t) \sim t^{2 D_{0}^{E}}$. (a) The Harper model (b) The Fibonacci model for three different values of the potential $V=0.5,1,1.5$ (from up to down).

is given by a quasi-periodic sequence for $0 \leq n \leq L$ and $V_{n}=0$ for $n<0$. We impose Dirichlet boundary condition at the edge $\psi_{L+1}=0$. Therefore, for $n \leq 0$, scattering states of the form $\psi_{n}=e^{i k n}+S e^{-i k n}$ represent the superposition of an incoming and a reflected plane wave. Here, $k=\arccos (E / 2)$ is the wave vector supported at the leads. Since there is only backscattering, the scattering matrix $S(E)=e^{i \Phi(E)}$ is of unit modulus and the total information about the scattering is contained in the phase $\Phi(E)$.

Using this setup we study various important quantities describing the open system in the thermodynamic limit $(L \rightarrow \infty)$. The main findings of our investigation can be summarized as follows. The resonance widths distribution $\mathcal{P}(\Gamma)$, the distribution of the Wigner delay 
times $\mathcal{P}(\tau)$, the $S$-matrix autocorrelation function $C(\chi)$ and the survival probability $P(t)$ have a power law behavior

$$
\begin{aligned}
\mathcal{P}(\Gamma) & \sim \Gamma^{-\alpha} ; \alpha=1+D_{0}^{E} \\
\mathcal{P}(\tau) & \sim \tau^{-\mu} ; \mu=2-D_{0}^{E} \\
P(t) & \sim t^{-\nu} ; \nu=1-D_{0}^{E} \\
C(\chi) & \sim 1-\chi^{\nu} .
\end{aligned}
$$

The important result here is the fact that the corresponding power law exponents are related to the fractal dimension of the spectrum in a very simple way.

\subsection{Statistics of resonances}

The poles of the $S$-matrix are associated with the eigenvalues of the non-Hermitian effective Hamiltonian (see Appendix (A.1)):

$$
H_{e f f}^{(L)}(E)=H^{(L)}+\mathrm{e}^{i k} \vec{e} \bigotimes \vec{e}=\left(\begin{array}{cccccc}
V_{1}+\mathrm{e}^{i k} & 1 & 0 & 0 & \cdots & 0 \\
1 & V_{2} & 1 & 0 & \cdots & 0 \\
\ldots \ldots & \ldots & \ldots & \ldots & \ldots \\
0 & 0 & \cdots & 0 & 1 & V_{L}
\end{array}\right),
$$

where $\vec{e}=(1,0,0, \ldots, 0)^{T}$ is an $L$-dimensional vector describing the sites at which the sample is coupled to the leads. Since $k=\arccos (E / 2)$ changes only slightly in the center of the band, we put $E=0$ and neglect the energy dependence of $H_{\text {eff }}$. The poles $\mathcal{E}_{n}=E_{n}-\frac{i}{2} \Gamma_{n}$ of the $S$-matrix are computed by direct diagonalization of $H_{\text {eff }}$ and the resonance widths $\Gamma_{n}$ are extracted by taking its imaginary parts. We note here that numerical diagonalization of complex non-Hermitian matrices is a time consuming process and imposes limitations on the system size due to limited storage capacity. The size of the matrices that we used in our analysis below was up to rank 5000 .

First, we will investigate the statistical distribution of the resonance widths $\Gamma$ for the Harper model at the critical point $\lambda=2$. Specifically we determine the integrated distributions

$$
\mathcal{P}_{\text {int }}(x)=\int_{x}^{\infty} \mathcal{P}\left(x^{\prime}\right) d x^{\prime}
$$

whose derivatives $\mathcal{P}(x)=-d \mathcal{P}_{\text {int }} / d x$ determine the probability density of resonance widths $\mathcal{P}(x=\Gamma)$. In all our calculations we take approximants of the golden mean $\sigma_{G}=(\sqrt{5}-$ $1) / 2$. For this case it is known that $D_{0}^{E} \approx 0.5[128,129]$.

Figure 4.5 shows $\mathcal{P}_{\text {int }}(\Gamma)$ for three different rational approximants $\sigma$ of the golden mean $\sigma_{G}$. It clearly displays an inverse power law

$$
\mathcal{P}_{\text {int }}(\Gamma) \sim \Gamma^{1-\alpha}
$$




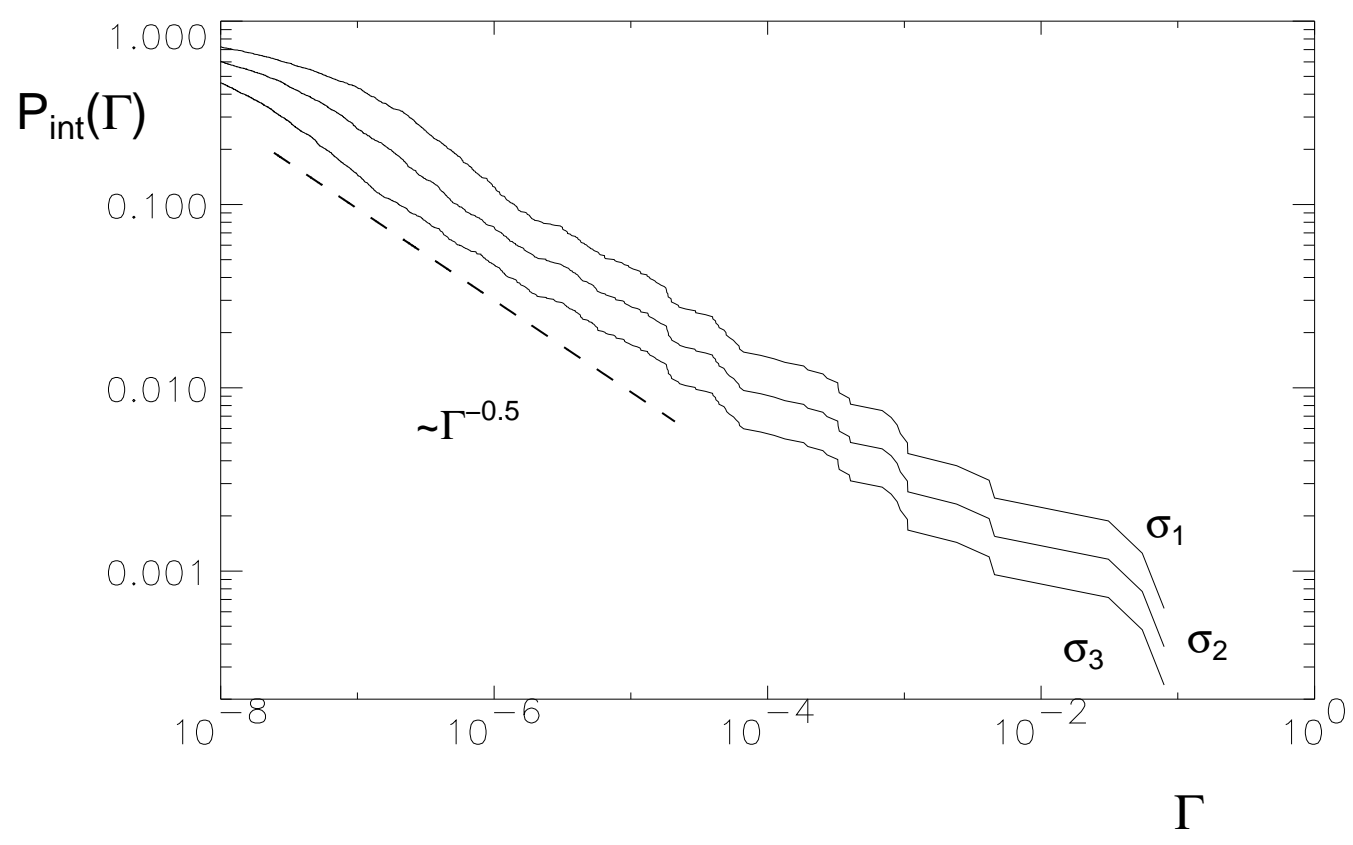

Figure 4.5: $\mathcal{P}_{\text {int }}(\Gamma)$ of the Harper model $(\lambda=2)$ for three approximants of $\sigma_{G}, \sigma_{1}=\frac{987}{1597} ; \sigma_{2}=$ $\frac{1597}{2584}$; and $\sigma_{3}=\frac{2584}{4181}$. An inverse power law $P_{\text {int }}(\Gamma) \sim \Gamma^{1-\alpha}$ is evident. A least squares fit yields $\alpha \approx 1.5$ in accordance with $D_{0}^{E} \simeq 0.5$ and Eq. (4.11).As is seen the lower cutoff of the scaling region decreases for higher approximants.

and thus the resonance width distribution behaves as stated in (4.11) with $\alpha \simeq 1.5=$ $1+D_{0}^{E}$. The integrated resonance width distribution cuts off at a small value of $\Gamma$ 's (see Fig. 4.5), since for all rational approximants of $\sigma_{G}$ the total number of $\mathcal{E}_{n}$ is finite. This cutoff, however, can be shifted to arbitrarily small values for higher approximants.

The connection between the exponents $\alpha$ and the fractal dimension $D_{0}^{E}$ of the spectrum of the closed system calls for an argument for its explanation. The following heuristic argument, similar in spirit to the one used in Section 3.3.2, provides some understanding of the power law (4.11). We consider successive rational approximants $\sigma_{i}=p_{i} / q_{i}$ of the continued fraction expansion of $\sigma$. On a length scale $q_{i}$ the periodicity of the potential is not manifested and the variance of a wave packet spreads as $\operatorname{var}(t) \sim t^{2 D_{0}^{E}}$ (Section 4.1.3). We attach the lead at the end of the segment $q_{i}$ which results in broadening the energy levels by a width $\Gamma$. The maximum time needed for a particle to recognize the existence of the leads, is $\tau_{q_{i}} \sim q_{i}^{1 / D_{0}^{E}}$. The latter is related to the minimum level width $\Gamma_{q_{i}} \sim 1 / \tau_{q_{i}}$. The number of states living in the interval is $\sim q_{i}$. Thus the number of states with resonance widths $\Gamma>1 / \tau_{q_{i}}$ is $\sim q_{i}$. Therefore $\mathcal{P}_{\text {int }}\left(\Gamma_{q_{i}}\right) \sim q_{i} \sim \Gamma^{-D_{0}^{E}}$. By repeating the same argument for higher approximants $\sigma_{i+1}=p_{i+1} / q_{i+1}$ we conclude that in the thermodynamic limit $\mathcal{P}(\Gamma) \sim \Gamma^{-\left(1+D_{0}^{E}\right)}$, in agreement with (4.11). Although the numerical results support the validity of the above argument, a rigorous mathematical proof is still lacking. 
The validity of the heuristic arguments (and thus of Eq. (4.11)) can be verified in a more general way in the case of the Fibonacci chain model where other scaling exponents can be obtained. We again find inverse power laws for the integrated distributions $\mathcal{P}(\Gamma)$. Here the exponent depends on the potential strength $V$, while Eq. (4.11) still relate the corresponding statistics to the fractal dimension $D_{0}^{E}$. Our results for various $V$ values are summarized in Fig. 4.6 and show a nice agreement between the exponents $\alpha$ and $D_{0}^{E}$ according to Eq. (4.11).

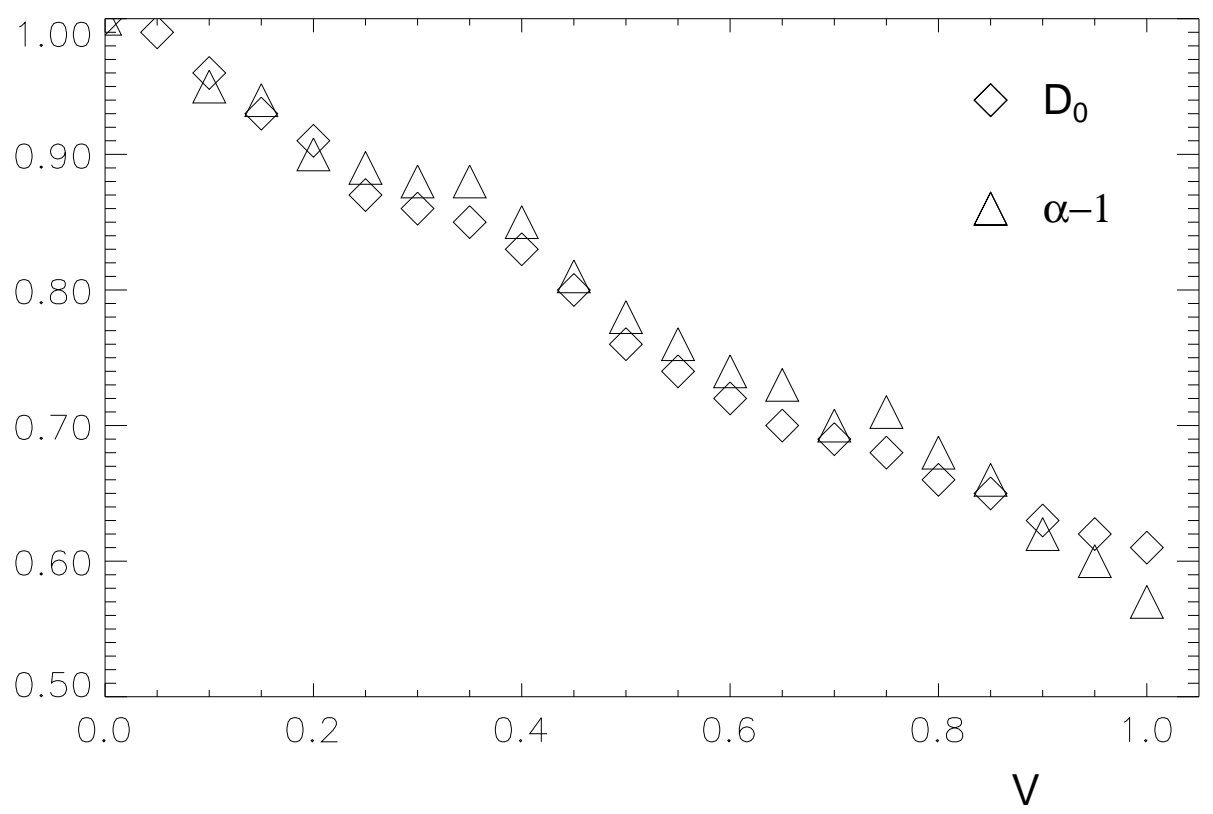

Figure 4.6: Power law exponents $\alpha$ (plotted as $\alpha-1$ ) of the resonance widths as a function of the potential strength $V$ for the Fibonacci model compared with the fractal dimension $D_{0}^{E}$ of the spectrum.

\subsection{Wigner delay times}

In this section we investigate the delay time statistics $\mathcal{P}(\tau)$. For its calculation we use recursion relations (2.15) and (2.25) developed in the previous chapter.

Our first observation is the fact that the Wigner delay time as a function of the energy shows clear self-similar behavior at all energy scales ( Fig. 4.7). This self-similarity appears due to the fractal nature of the real parts of the poles of the $S$-matrix (see Eq. (4.19) and the discussion afterwards). Moreover the numerical analysis shows that the positions of the real part of the poles are very similar to the eigenenergies of the corresponding closed system and their fractal dimensions are approximately the same. 

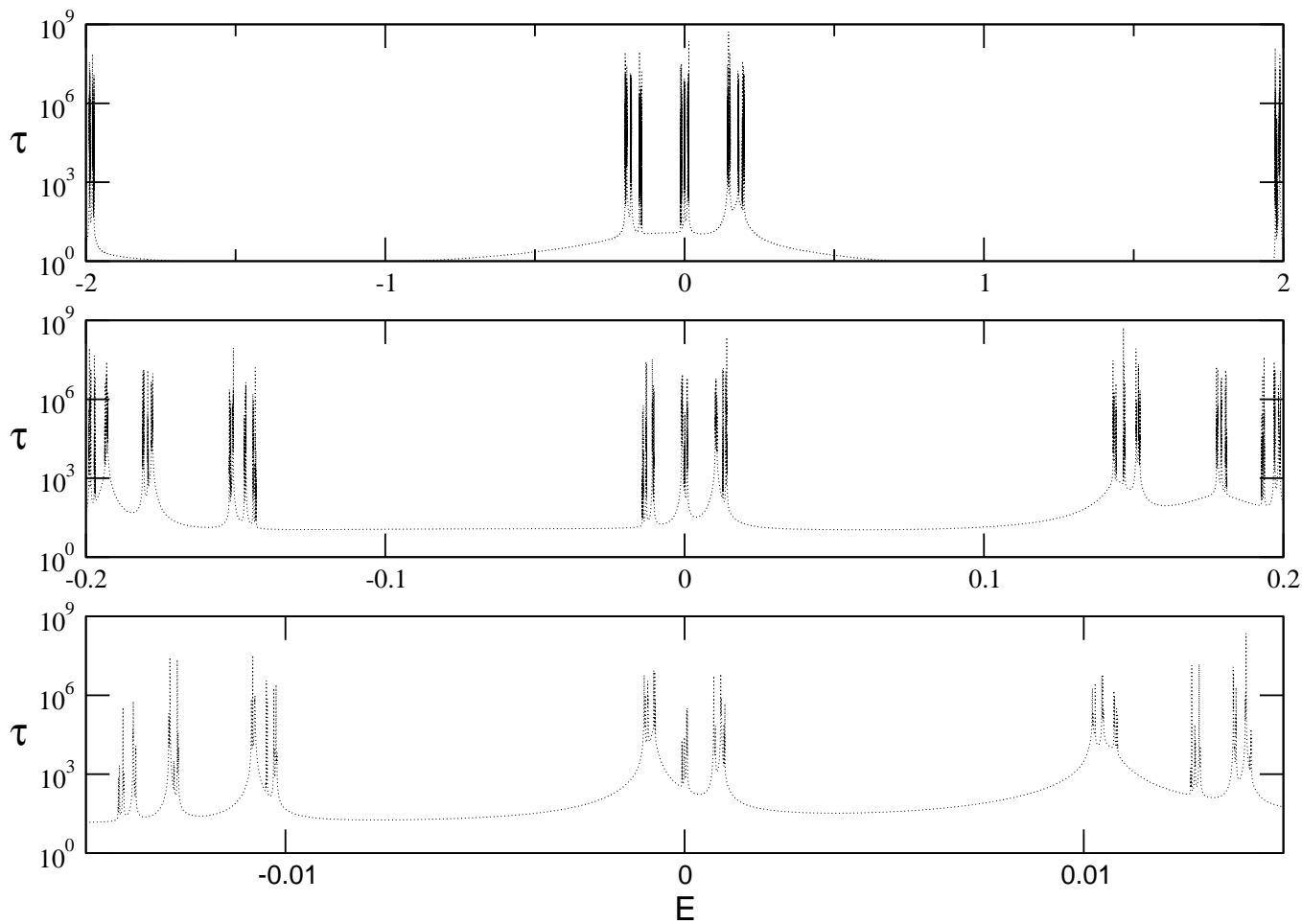

Figure 4.7: The Wigner delay time as a function of energy for the Harper model shown at three different energy scales.

In Fig. 4.8 we report the integrated $\mathcal{P}_{\text {int }}(\tau)$ for three different rational approximants of the golden mean. Due to the efficiency of our recursion relation (2.25) we can approximate $\sigma_{G}$ by increasing the periodicity $q$ of the potential as much as we like. Our numerical data are compatible with an inverse power law i.e.

$$
\mathcal{P}_{\text {int }}(\tau) \sim \tau^{1-\mu}
$$

with a value of $\mu \approx 1.5=2-D_{0}^{E}$ given by a best least square fit, in agreement with Eq. (4.12).

The following argument allows us to understand the relation between the power law decay exponent $\mu$ and the fractal dimension $D_{0}^{E}$. Our starting point is the well known relation 


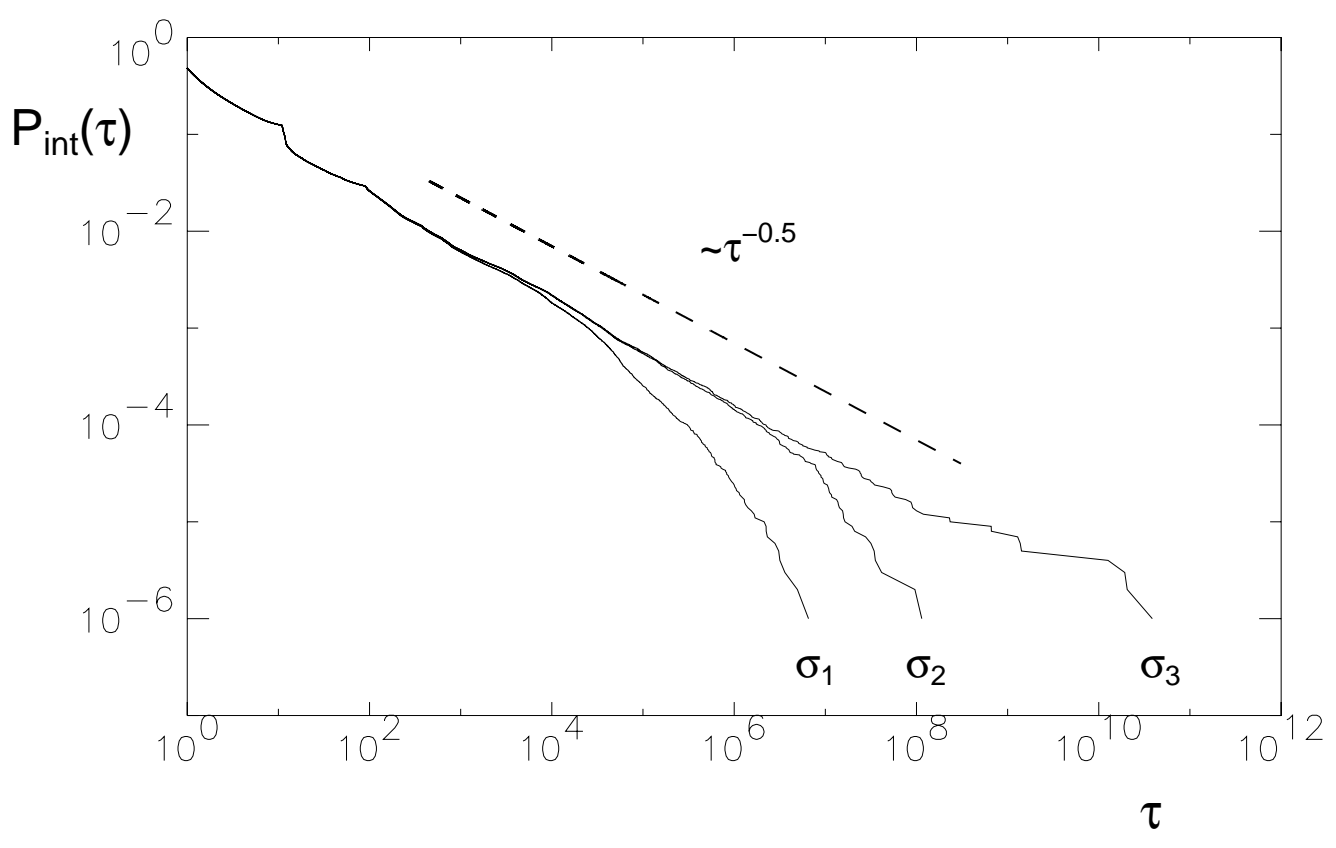

Figure 4.8: $\mathcal{P}_{\text {int }}(\tau)$ of the Harper model $(\lambda=2)$ for three approximants of the golden mean $\sigma_{1}=\frac{233}{377} ; \sigma_{2}=\frac{987}{1597} ;$ and $\sigma_{3}=\frac{832040}{1346269}$. An inverse power law $P_{\text {int }}(\tau) \sim \tau^{1-\mu}$ is evident. A least squares fit yields $\mu \approx 1.5$ in accordance with $D_{0}^{E} \simeq 0.5$ and Eq. (4.12). As is seen the upper cutoff of the scaling region increases for higher approximants.

(see Eq. (A.26) in Appendix A.2)

$$
\tau(E)=\sum_{n=1}^{L} \frac{\Gamma_{n}}{\left(E-E_{n}\right)^{2}+\Gamma_{n}^{2} / 4}
$$

which connects the Wigner delay times and the poles of the $S$-matrix. It is evident that anomalously large time delay $\tau(E) \sim \Gamma_{n}^{-1}$ corresponds to the cases when $E \simeq E_{n}$ and $\Gamma_{n} \ll 1$. In the neighborhood of these points, $\tau(E)$ can be approximated by a single Lorentzian (4.19). Sampling the energies $E$ with step $\Delta E \ll \Gamma_{\min }$ we calculate the number of points for which the time delay is larger than some fixed value $\tau$ (see Fig. 4.9). Assuming that the contribution of each Lorentzian is proportional to its width one can estimate this number as $\sum_{\Gamma_{n}<1 / \tau} \Gamma_{n} / \Delta E$. For the integrated distribution of delay times we obtain $\mathcal{P}_{\text {int }}(\tau) \sim \int^{1 / \tau} d \Gamma \mathcal{P}(\Gamma) \Gamma \sim \tau^{-(2-\alpha)}$ in the limit $\Delta E \rightarrow 0$ where we used the small resonance width asymptotic given by Eq. (4.12) (for similar argumentation see also [8,132]). Then for the asymptotic distribution of delay times we get $\mathcal{P}(\tau) \sim \tau^{-\left(2-D_{0}^{E}\right)}$ in agreement with $(4.12)$ and our numerical findings.

Different power law exponents obtained for Fibonacci chain model for various values of the potential $V$ are presented in Fig. 4.10. The comparison with the fractal dimension $D_{0}^{E}$ of the spectrum confirms again the validity of Eq. (4.12). 


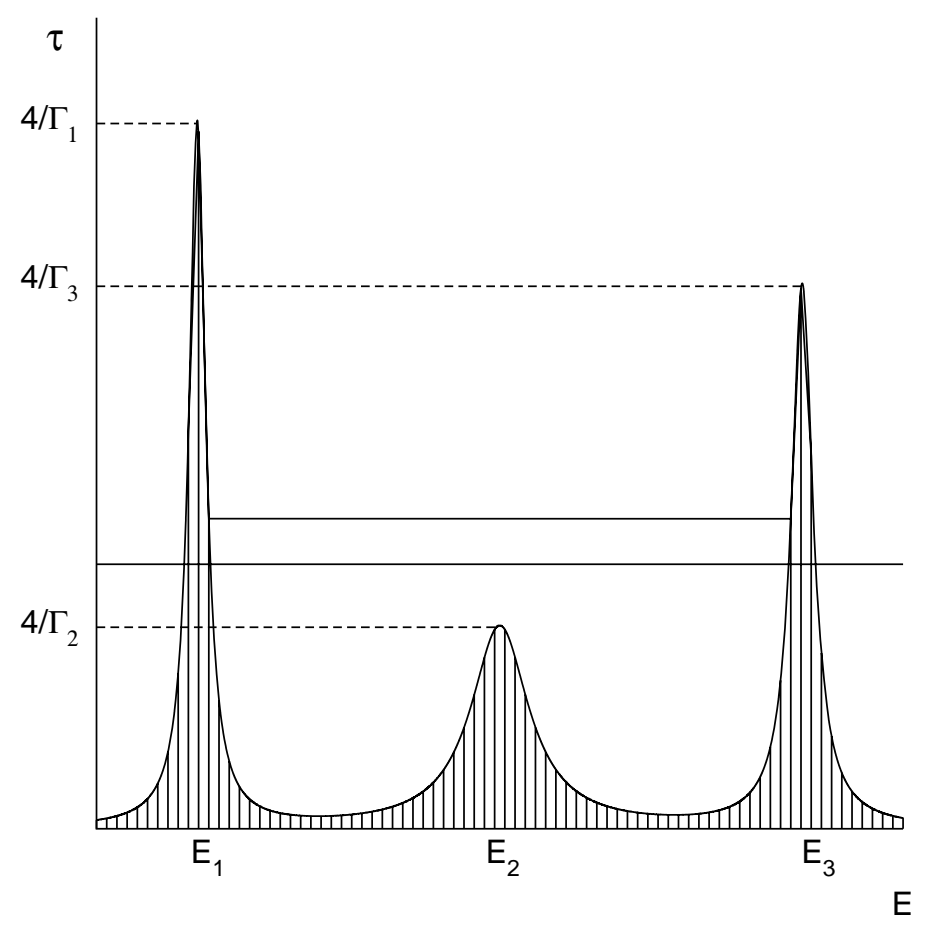

Figure 4.9: Schematic plot for the Wigner delay time as a function of energy according to Eq. (4.19)

\subsection{Survival probability}

A complementary approach for the study of open systems is to consider the time evolution of an excitation originally started inside the system. Then one of the most important quantities characterized this process is the survival probability. It is defined as the probability to find a particle localized initially at $t=0$ inside the open system to be found there after a time $t$.

The dynamics of the quantum mechanical particle is described by the time-dependent Schrödinger equation

$$
i \frac{d \psi_{n}(t)}{d t}=V_{n} \psi_{n}(t)+\psi_{n+1}(t)+\psi_{n-1}(t)
$$

corresponding to the tight-binding Hamiltonian (4.9). We assume absorbing boundary conditions at the ends of the sample (our results are valid also for the case of a semi- 


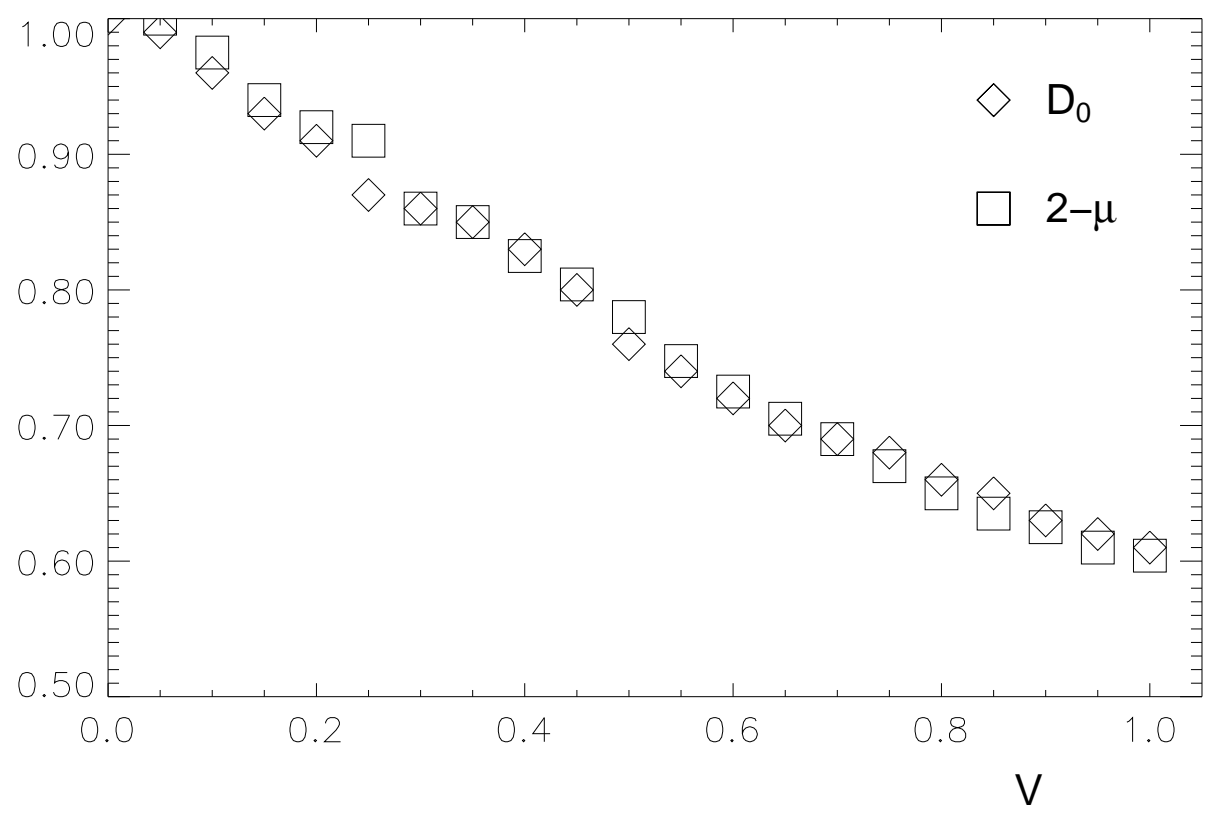

Figure 4.10: Power law exponents $\mu$ (plotted as $2-\mu$ ) of the delay times as a function of the potential strength $V$ for the Fibonacci model compared with the fractal dimension $D_{0}^{E}$ of the spectrum.

infinite lattice with one absorbing boundary) which correspond to a perfect coupling with the outside. The initial excitation is a $\delta$-like packet launched at one of the boundaries, i.e. $\psi_{n}(t=0)=\delta_{n, 1}$. Equation (4.20) has been integrated numerically using a Cayley scheme [133] with integration time step $d t=0.1$. We attached 15 additional sites at the ends of the sample and erased all components of the wave packet on these sites after each time step $d t$. The decay of the norm of the wave packet obtained in this way was not affected by a further decrease of $d t$.

We investigate first the survival probability $P(t)$ for the Harper model at the critical point $\lambda=2$ and $\sigma=\sigma_{G}$. The results for various sample lengths $L$ are shown in Fig. 4.10. In all cases the survival probability clearly displays an inverse power law

$$
P(t) \equiv \sum_{n=1}^{L}\left|\psi_{n}(t)\right|^{2} \sim t^{-\nu} .
$$

The best fit to the numerical data yields $\nu=0.55 \pm 0.05$ in accordance with Eq. (4.13).

For a further test of the validity of Eq. (4.13) we now turn to the Fibonacci model. In Fig. 4.12 we report some of our numerical results for $P(t)$. Again we find a power-law decay $P(t) \sim t^{-\nu}$, where the exponent depends on the potential strength $V$. The exponents $\nu$ extracted for various $V$ are compared with the corresponding fractal dimension $D_{0}^{E}$ in 


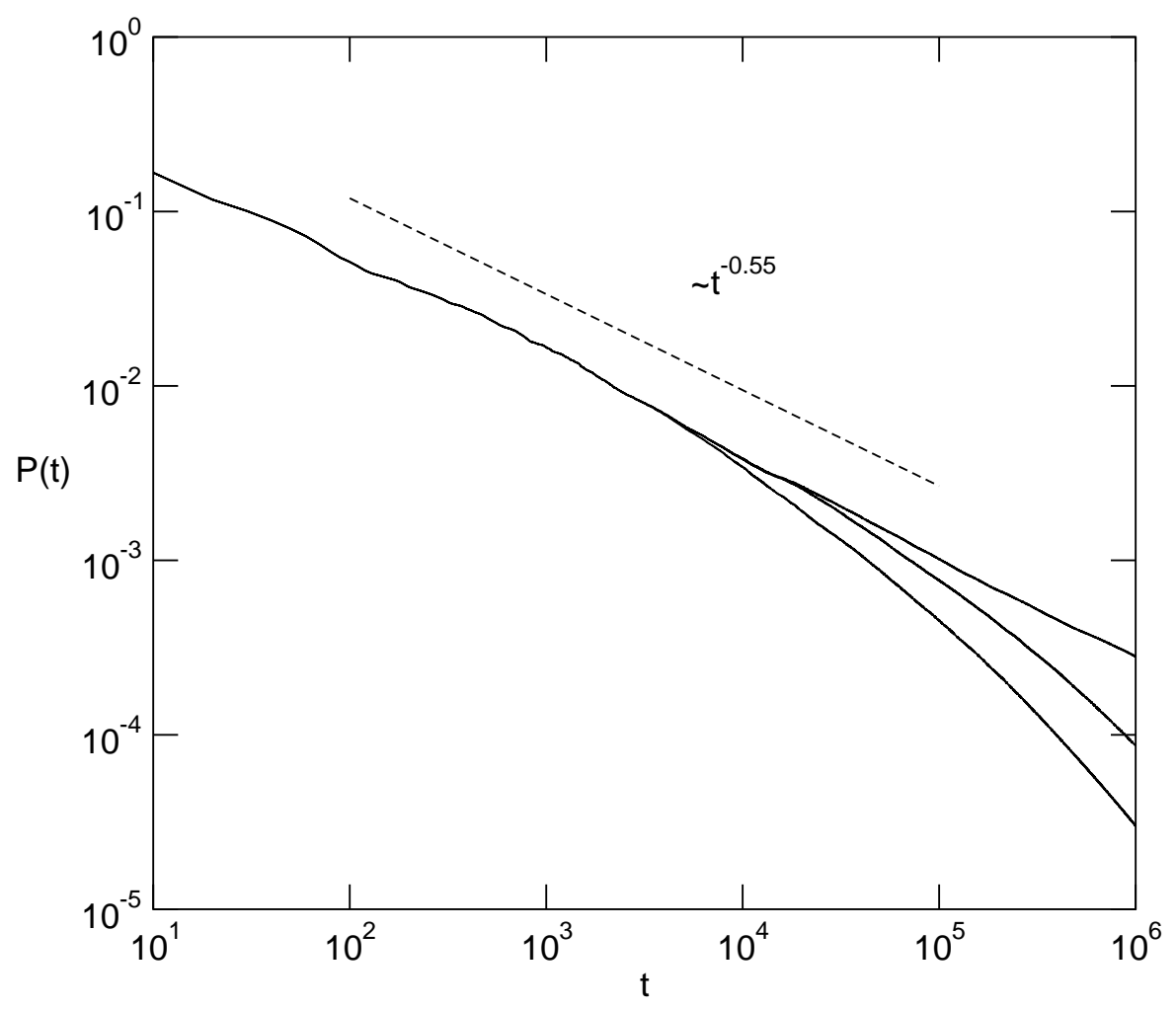

Figure 4.11: The survival probability $P(t)$ of the Harper model $(\lambda=2)$, for three different sample lengths $L=250,500,4000$ exhibits an inverse power-law $P(t) \sim t^{-\nu}$. A least squares fit yields $\nu=0.55 \pm 0.05$ in accordance with $D_{0}^{E} \simeq 0.5$ and Eq. (4.13).

Fig. 4.13 and confirm the validity of Eq. (4.13).

We now want to give a general argument for the validity of Eq. (4.13). As we know from section 4.3 the open system can be described by the effective Hamiltonian (4.15) (here we neglect its energy dependence at the band center):

$$
H_{e f f}^{(L)}(E)=H^{(L)}-i \vec{e} \bigotimes \vec{e}
$$

where $\vec{e}=(1,0,0, \ldots, 0)^{T}$ is an $L$-dimensional vector, that describes at which site we impose the absorbing boundary condition. The eigenenergies of the effective Hamiltonian are complex $\mathcal{E}_{k}=E_{k}-i \Gamma_{k} / 2$ leading to the decay of the survival probability $P(t)$. When the on-site potential fulfills $\left|V_{n}\right|>1$ the imaginary shift can be considered as a small perturbation of the Hamiltonian of the closed system. In this case according to the perturbation 


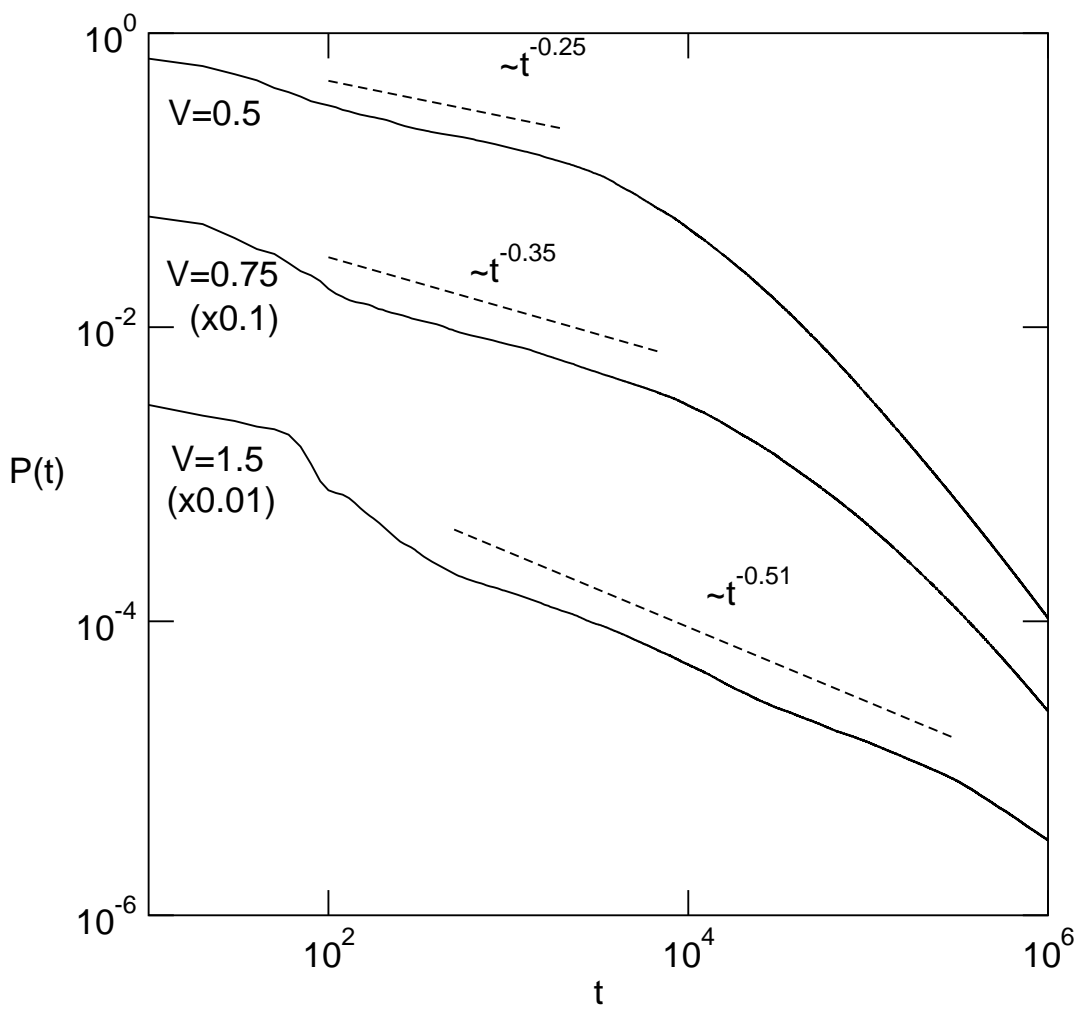

Figure 4.12: Survival probabilities $P(t)$ of the Fibonacci model, for three different potential strengths $V_{1}=0.5, V_{2}=0.75$ and $V_{3}=1.5$ showing inverse power-laws $P(t) \sim t^{-\nu}$ (dashed lines). The sample size is $L=2000$ in all cases.

theory, $\Gamma_{k} \sim\left|\psi_{1}^{k}\right|^{2}$ holds, where $\psi_{n}^{k}$ is the $n$-th component of an eigenstate of the closed system with energy $E_{k}$. The survival probability is then given by

$$
P(t) \simeq \sum_{k}\left|c_{k}\right|^{2} e^{-\Gamma_{k} t}
$$

where $c_{k}$ are overlapping elements of the initial state with the eigenstates $\psi_{n}^{k}$.

Now choosing the initial state to be concentrated at site $n=1$, we have that the overlapping elements $\left|c_{k}\right|^{2}$, appearing in Eq. (4.23), are equal to the $n=1$ component of the eigenstates i.e. $\left|c_{k}\right|^{2}=\left|\psi_{1}^{k}\right|^{2}$. The latter, however are proportional to the resonances widths (see Fig. 4 ) and thus we get that $\left|c_{k}\right|^{2} \sim \Gamma_{k}$. Using this and converting the sum (4.23) into an integral 


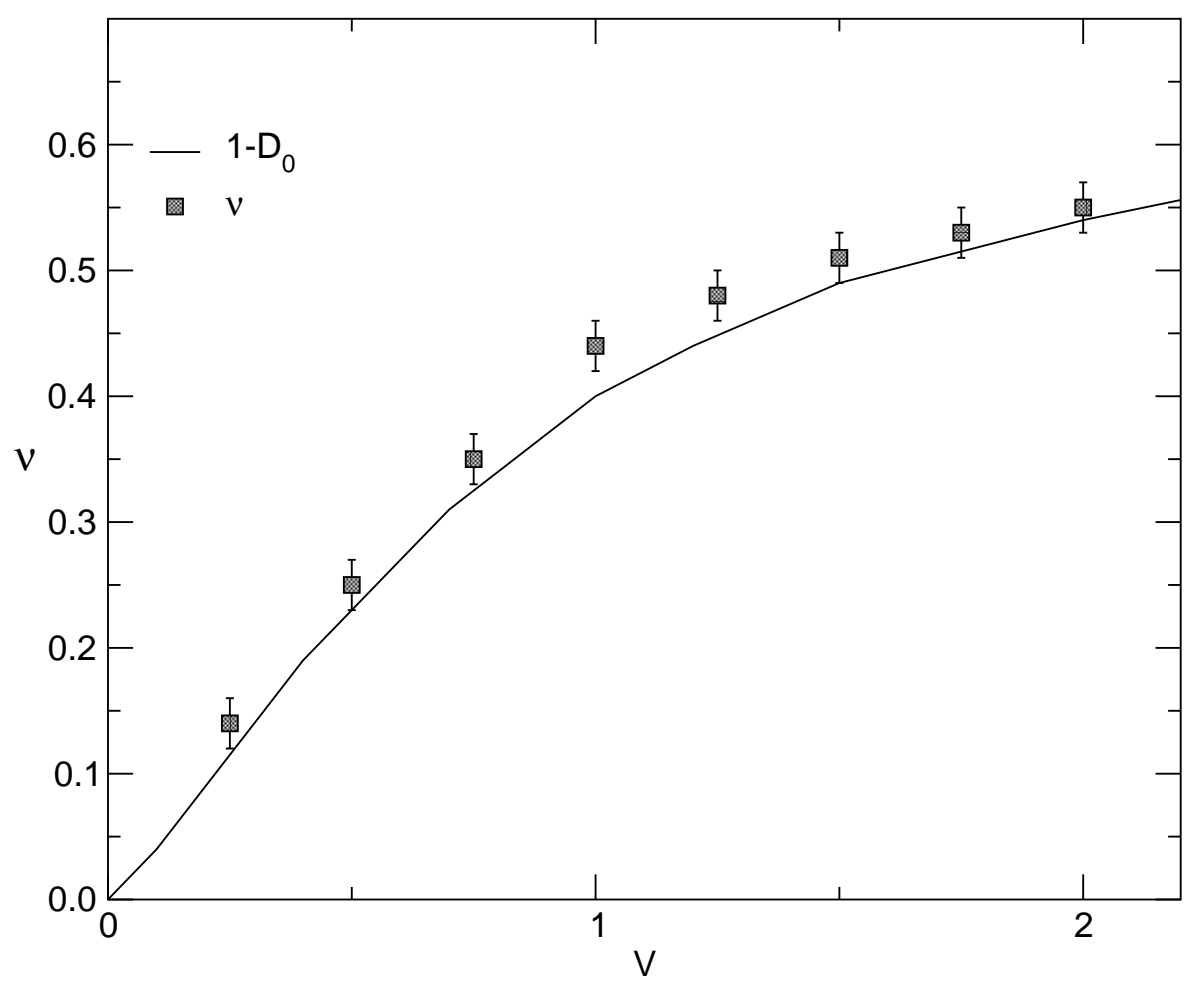

Figure 4.13: Power-law exponents $\nu$ of the survival probability obtained numerically as a function of the potential strength $V$ for the Fibonacci model. The solid line is the theoretical prediction $\nu=1-D_{0}^{E}$.

we obtain:

$$
P(t) \sim \int \Gamma \mathcal{P}(\Gamma) e^{-\Gamma t} d \Gamma
$$

where $\mathcal{P}(\Gamma)$ is the resonance width distribution. In order to check the validity of the perturbative arguments resulting in Eq. (4.24), we numerically calculate the resonance widths $\Gamma_{k}$ and the overlapping elements $c_{k}$ for the Harper and Fibonacci models. As can be seen from Fig. 4.14 the prediction $\left|c_{k}\right|^{2} \sim \Gamma_{k}$ of the perturbation theory holds even for small $V$, although the coupling of the whole system to the exterior is not weak, but is a perfect coupling. Moreover the wavefunctions of the closed analog are not localized, but have self-similar structure on all scales and may be expected to undergo severe changes when the system is opened in that way.

Thus, Eq. (4.24) allows us to calculate within perturbation theory the decay of the survival 


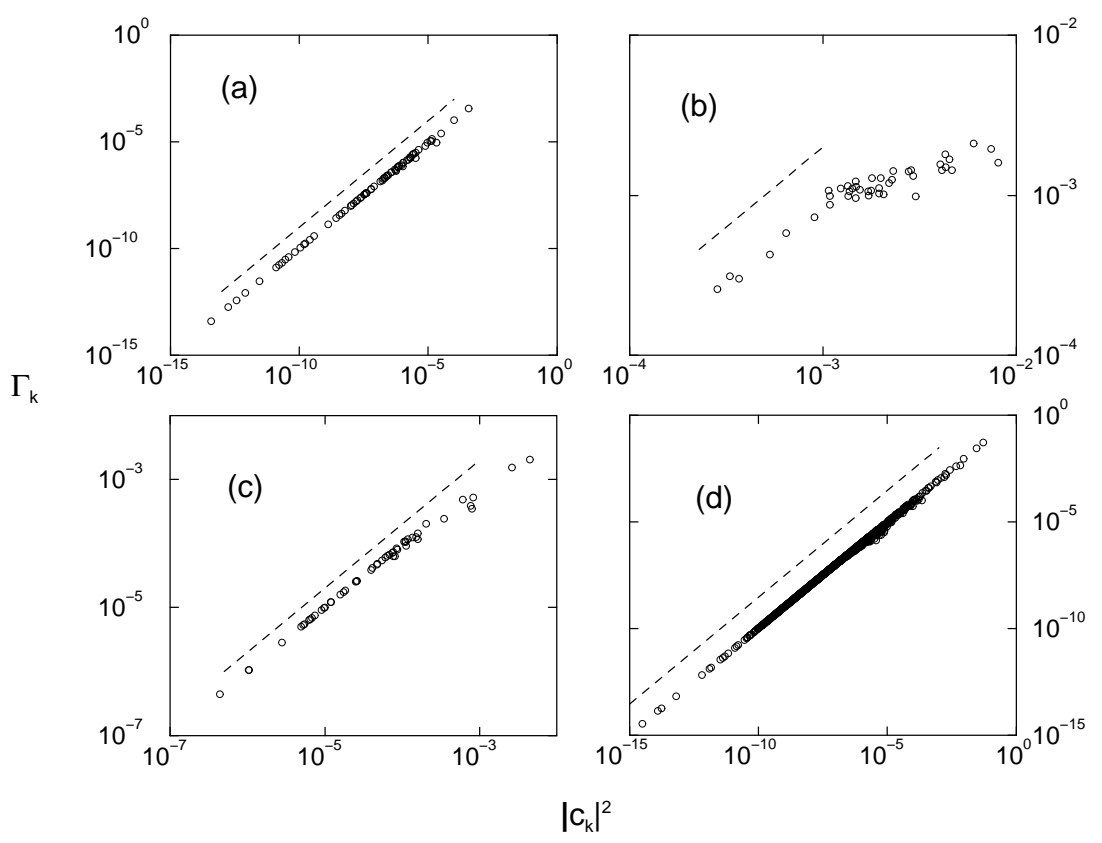

Figure 4.14: Resonance widths $\Gamma_{k}$ as a function of the overlapping elements $\left|c_{k}\right|^{2}$. The data are obtained by direct diagonalization of the effective Hamiltonian $\mathcal{H}_{\text {eff }}$ (see Eq. (4.22)). The dashed lines of slope 1 are shown for comparison demonstrating a linear relation for small $\Gamma$. In all cases the sample size is $L=1597$, corresponding to an approximant of the golden mean $\sigma=\frac{987}{1597}$. (a) Harper model for $\lambda=2$; (b) Fibonacci model for $V=0.1$; (c) Fibonacci model for $V=0.5$; and (d) Fibonacci model for $V=1.5$.

probability, provided that the resonance widths distribution $\mathcal{P}(\Gamma)$ is known. Using our previous result for $\mathcal{P}(\Gamma)$ Eq. (4.11) we find the asymptotic power-law decay for the survival probability $P(t)$ stated in Eq. (4.13).

For finite samples the power-law decay of $P(t)$ (Eq. (4.13)) holds up to a break time $t^{*}$, beyond which it turns into an exponential decay. The rate of the latter is determined by the smallest resonance width $\Gamma_{\min }$ and thus $t^{*} \sim 1 / \Gamma_{\min }$. An estimation for the scaling properties of $t^{*}$ with respect to $D_{0}^{E}$ and the size of the system $L$ can be derived as follows. Imposing the normalization condition for the resonance width distribution and assuming that the power law (4.11) for $\mathcal{P}(\Gamma)$ is valid for $\Gamma \geq \Gamma_{m i n}$, we get

$$
L=\int_{\Gamma_{\min }}^{\infty} d \Gamma \mathcal{P}(\Gamma)=\frac{1}{\Gamma_{\min }^{D_{0}^{E}}} .
$$

Solving the above equation with respect to $\Gamma_{\min }$ and substituting in the previous expression $t^{*} \sim 1 / \Gamma_{\min }$ we obtain

$$
t^{*} \sim L^{\eta} ; \quad \eta=1 / D_{0}^{E},
$$

This prediction is verified numerically in Fig. 4.15 where we defined $t^{*}$ as the time where 
$P(t)$ deviates by $5 \%$ from the power-law decay. We want to point out that the increase of $t^{*}$ for decreasing $D_{0}^{E}$ is consistent with the enlargement of the interval where $\left|c_{k}\right|^{2} \sim \Gamma_{k}$ holds and its shift towards smaller values (note the change of the axes scales in Figs. 4.14 (b)$4.14(\mathrm{~d}))$.

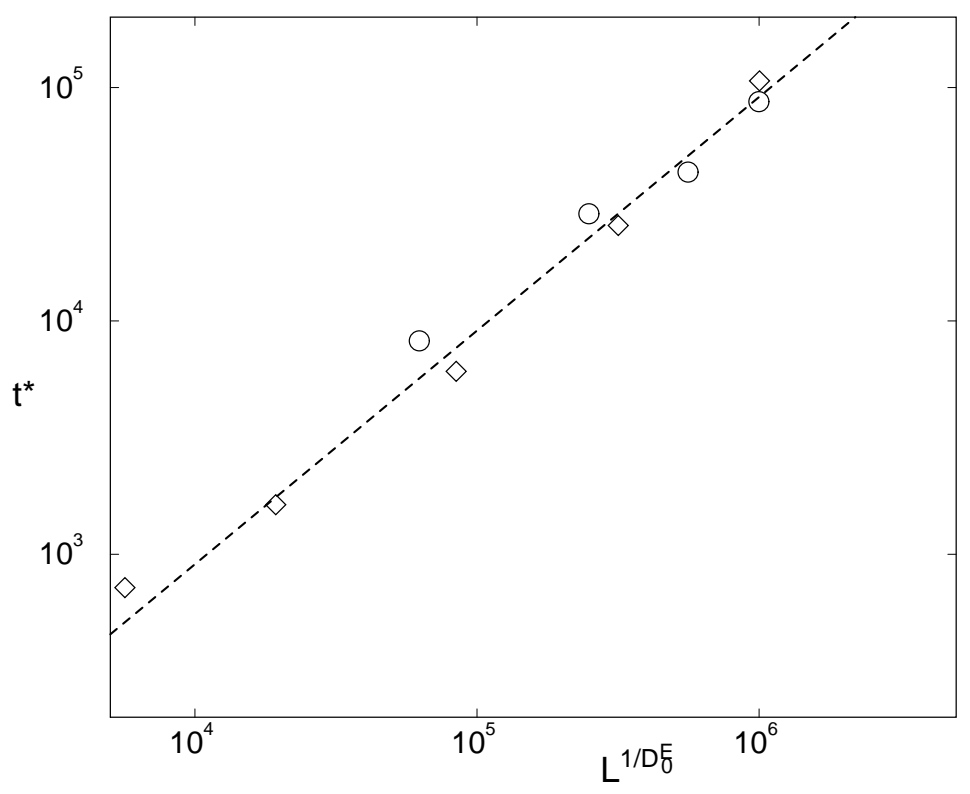

Figure 4.15: Dependence of the break-time $t^{*}$, on the system size $L$ and the box-counting dimension $D_{0}^{E}$. ( ( ) refer to the Harper model at $\lambda=2$ and various $L$ 's while $(\diamond)$ refer to the Fibonacci model with various $V$ 's and $L=2000$. The dashed line has slope 1 and corresponds to the theoretical expectation Eq. (4.26).

\subsection{S-matrix autocorrelation function}

An immediate consequence of Eq. (4.13) is the fact that the scattering matrix autocorrelation function $C(\chi) \equiv<S(E)^{\dagger} S(E+\chi)>_{E}$ decays in a power law fashion. In particular, using the relation between the survival probability $P(t)$ and $C(\chi)$ [134], we obtain

$$
1-C(\chi) \sim \chi \int d t P(t) \exp (-i t \chi) \sim \chi^{\nu}, \quad \chi \ll 1 .
$$

Equation (4.27) is in contrast to the Lorentzian form of $C(\chi)$ predicted by RMT for chaotic/ballistic systems [135]. Comparison of $C(\chi)$ for the Harper model at $\lambda=2$ and for various $V$-values of the Fibonacci model with the theoretical prediction Eq. (4.27) in Fig. 4.16 shows a nice agreement and provides an additional check for the validity of Eq. (4.13). In these calculations the sample of the length equal to $L=10946$ is attached to one lead. The phase of the corresponding scattering matrix $S(E)=e^{i \Phi(E)}$ is calculated with the help of the iteration relation (2.15). 

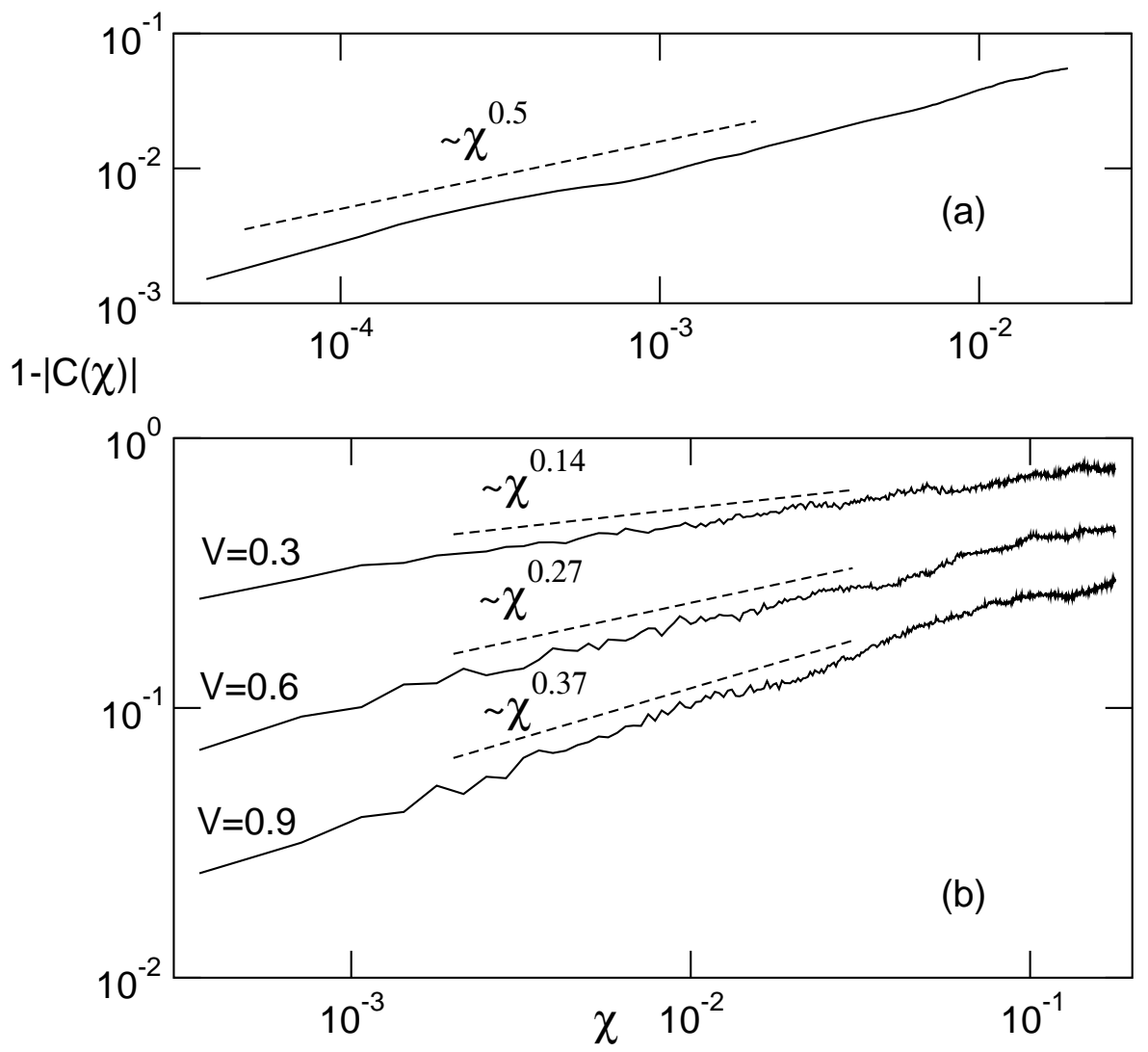

Figure 4.16: The scattering autocorrelation function Eq. (4.27) (plotted as $1-|C(\chi)|$ ) for (a) the Harper model $(\lambda=2)$ and (b) for some representative $V$ values of the Fibonacci model. The dashed lines are the theoretical expectations (4.27).

\subsection{Outlook}

It has been shown that various statistical quantities, describing open one-dimensional quasiperiodic systems at critical conditions, decay algebraically. The corresponding exponents are determined by the fractal (box-counting) dimension of the spectrum of the closed system. These results are obtained numerically for two different types of quasi-periodic tight-binding models and are supported by analytical arguments.

The rigorous mathematical treatment of these problems is up to now still lacking. In 
particular, it would be interesting to understand the role of the other fractal dimensions of the spectrum and eigenstates in the properties of the open system. This task is however a very difficult one, because even for the closed systems there are only few results proven rigorously.

An experimental verification of our predictions is also desired. A possible experiment can be realized with microwaves propagating through an array of scatterers arranged in a quasiperiodic sequence. This type of experiments have been already done successfully for closed systems, and their extension to open geometries is realistic. 


\section{Chapter 5}

\section{Conclusion}

This thesis deals with the statistical properties of various open mesoscopic systems. In particular, different disordered, chaotic and quasi-periodic systems at criticality are studied. All of them possess dynamical or spectral properties, which can not be captured by the random matrix theory. The main quantities under consideration are distributions of the resonance widths and Wigner delay times, which are fundamental for the quantum description of open systems and are motivated by experimental applications. In some cases other characteristics like the survival probability and statistics of the phases of the $S$-matrix are investigated as well. The results of this study demonstrate how the generic properties of the corresponding closed systems like localization, diffusion or fractality manifest themselves in the statistical properties of the open counterparts.

We start our analysis with the one-dimensional Anderson model, which is one of the basic models in the localization theory. The first quantity we study is the reflection phase of the $S$-matrix. Usually it is assumed that this quantity is distributed uniformly. This assumption is very important for the single parameter scaling hypothesis - the cornerstone of the localization theory. Our investigation reveals however that the distribution of the phases is strongly nonuniform for strong disorder. The same is true in the case of weak disorder for special values of the wave vector related to the discrete nature of the lattice and for energies near the band edges. In all this cases the violation of the single parameter scaling hypothesis is expected. The second quantity under consideration is the Wigner delay time. We find that the typical delay time corresponds to the propagation of the particle at the distance of the order of the localization length. At the same time the mean value of the delay time diverges, when the system size goes to infinity, because of the long algebraic tail of the distribution. The existence of this tail is independent of the nature of disorder and is believed due to the presence of the Azbel resonances - the special disorder arrangements allowing the particle to traverse a very long distance before being reflected. We also discuss the distribution of the resonance widths and explain how it can be derived from the localization of the eigenfunctions. 
Next we study systems characterized by classical diffusion. The quantum properties of such systems are influenced by diffusion on one hand and by quantum localization effects on the other hand. The models we use are the kicked rotor, its two-dimensional generalization and their randomized versions. The kicked rotor model is one of the first models appeared in the field of quantum chaos. Its randomized version can be mapped to the disordered tight-binding equation. Thus our choice of the models allows us to study the problems in a way relevant both for the quantum chaos and disordered systems.

The first issue, we investigate in this framework, is the distribution of the eigenstates intensities of the closed system. For disordered systems the field theoretic methods predict the existence of prelocalized states, which appear due to some rear realizations of disordered potential and are responsible for the long tails in the distributions of various physical quantities. We show that such states do exist also for dynamical systems and they determine the tail of the distribution of the eigenfunction intensities. The analysis of this tail in the presence and in the absence of time reversal symmetry allows us to resolve the contradiction between two different theoretical predictions existing for disordered systems.

The second issue concerns the properties of the open system in the diffusive regime. Specifically, we study the distributions of the resonance widths and delay times. We find that the part of these distributions corresponding to the large values of the resonance widths and small values of the delay times is determined by the classical diffusion. The complementary parts of these distributions - small resonance widths and large delay times are strongly connected to the prelocalized states of the closed system. The effect of the breaking of the time reversal symmetry on these distributions is studied as well.

The last part of this thesis is devoted to the one-dimensional quasiperiodic systems at critical conditions. The first model, we consider, is the Harper model, which appeared originally for the description of the Bloch electron in the magnetic field. Despite of its onedimensional character, the Harper model exhibits a rich variety of interesting properties, e.g. the metal-insulator transition taking place at the critical point. At this point the spectrum is a Cantor set with non-trivial fractal dimension. The last property is shared by the Fibonacci chain model, used intensively in the field of quasicrystals. For both models we investigate the distribution of the resonance widths and the Wigner delay times, as well as the survival probability and the $S$-matrix autocorrelation function. The main conclusion of our study is the following. The behavior of these quantities can be described by different power laws with exponents, which are given essentially by the fractal (boxcounting) dimension of the energy spectrum of the corresponding closed system. 


\section{Appendix A}

\section{Effective Hamiltonian, resonances and Wigner delay time}

\section{A.1 Effective Hamiltonian for open one-dimensional system}

The aim of this appendix is to show that the poles of the $S$-matrix can be found as eigenvalues of some non-Hermitian effective Hamiltonian.

From Eq. (2.7) we know that the $S$-matrix (reflection coefficient $r$ in our case) can be written in terms of the total transfer matrix as:

$$
r=-\frac{P_{11}^{(L)}(E)+P_{12}^{(L)}(E) \mathrm{e}^{-i k}}{P_{11}^{(L)}(E)+P_{12}^{(L)}(E) \mathrm{e}^{i k}}
$$

Here $P_{i k}^{(L)}(E)$ are elements of the total transfer matrix of the system of size $L$. By real values of energy the nominator in the last expression is a complex conjugate to the denominator, so $|r|=1$ as expected. When we allow energy to take complex values then this is not true any more. Moreover for some values of energy the denominator can become equal to zero so that $r$ becomes infinite. These are the poles of the $S$-matrix and they are solutions of the following equation:

$$
P_{11}^{(L)}(E)+P_{12}^{(L)}(E) \mathrm{e}^{i k}=0
$$

In order to rewrite this equation using some effective Hamiltonian, let us first consider the closed system. The eigenenergies of the closed system with Dirichlet boundary conditions can be found as eigenvalues of the Hamiltonian:

$$
\operatorname{det}\left(H^{(L)}-E\right)=0
$$


where the Hamiltonian $H^{(L)}$ for a system of size $L$ is given by a $L \times L$ three-diagonal matrix:

$$
H^{(L)}=\left(\begin{array}{cccccc}
V_{1} & 1 & 0 & 0 & \cdots & 0 \\
1 & V_{2} & 1 & 0 & \cdots & 0 \\
\cdots & \ldots & \ldots & \ldots & \ldots & \ldots \\
0 & 0 & \cdots & 0 & 1 & V_{L}
\end{array}\right)
$$

The equation for the eigenenergies can be also written in terms of the total transfer matrix:

$$
\left(\begin{array}{c}
0 \\
\phi_{L}
\end{array}\right)=\left(\begin{array}{cc}
P_{11}^{(L)}(E) & P_{12}^{(L)}(E) \\
P_{21}^{(L)}(E) & P_{22}^{(L)}(E)
\end{array}\right)\left(\begin{array}{c}
\phi_{1} \\
0
\end{array}\right)
$$

where $\phi_{K}$ are components of the eigenfunction and we use Dirichlet boundary conditions at $k=0, L+1$. From this equation we conclude immediately that $P_{11}^{(L)}(E)=0$ if and only if $E$ is an eigenenergy, i.e.

$$
P_{11}^{(L)}(E)=0 \Leftrightarrow \operatorname{det}\left(H^{(L)}-E\right)=0
$$

Both expressions in the last equation are polynomials of the same order. Since they have the same roots they must be equal up to some constant factor. This factor can be found by comparison the coefficients at $E^{L}$, which is equal to 1 for $P_{11}^{(L)}(E)$ and equal to $(-1)^{L}$ for $\operatorname{det}\left(H^{(L)}-E\right)$. So we obtain

$$
P_{11}^{(L)}(E)=\operatorname{det}\left(E-H^{(L)}\right)
$$

Now using the definition of the total transfer matrix we have

$$
\left(\begin{array}{cc}
P_{11}^{(L)}(E) & P_{12}^{(L)}(E) \\
P_{21}^{(L)}(E) & P_{22}^{(L)}(E)
\end{array}\right)=\left(\begin{array}{cc}
P_{11}^{(L-1)}(E) & P_{12}^{(L-1)}(E) \\
P_{21}^{(L-1)}(E) & P_{22}^{(L-1)}(E)
\end{array}\right)\left(\begin{array}{cc}
E-V_{1} & -1 \\
1 & 0
\end{array}\right)
$$

where $P^{(L-1)}(E)$ is the transfer matrix corresponding to the chain of the size $L-1$ which is obtained from the initial chain of the size $L$ by removing the first site. In particular this equation yields

$$
P_{12}^{(L)}(E)=-P_{11}^{(L-1)}(E)=-\operatorname{det}\left(E-H^{(L-1)}\right) .
$$

Substituting expressions (A.7) and (A.9) in Eq. (A.2) one obtains

$$
\operatorname{det}\left(E-H^{(L)}\right)-\operatorname{det}\left(E-H^{(L-1)}\right) \mathrm{e}^{i k}=0,
$$

which is equivalent to the following equation

$$
\operatorname{det}\left(H_{e f f}^{(L)}(E)-E\right)=0,
$$


where the effective Hamiltonian is obtained from the Hamiltonian of the closed system by adding $\mathrm{e}^{i k}$ to its first diagonal element i.e.

$$
H_{e f f}^{(L)}(E)=H^{(L)}+\mathrm{e}^{i k} \vec{e} \bigotimes \vec{e}=\left(\begin{array}{cccccc}
V_{1}+\mathrm{e}^{i k} & 1 & 0 & 0 & \cdots & 0 \\
1 & V_{2} & 1 & 0 & \cdots & 0 \\
\ldots \ldots & \ldots & \ldots & \ldots & \ldots & \ldots \\
0 & 0 & \cdots & 0 & 1 & V_{L}
\end{array}\right)
$$

where $\vec{e}=(1,0,0, \ldots, 0)^{T}$ is an $L$-dimensional vector.

The obtained equation (A.12) for the effective Hamiltonian is a special case of the general expression emerging in the construction for the $S$-matrix $[7,8]$ :

$$
S=I-2 i \pi W^{\dagger} \frac{1}{E-H_{e f f}} W,
$$

where $W$ is a rectangular $M \times L$ matrix describing the coupling of the system to the "outside world", $M$ is the number of open channels and the effective Hamiltonian is given by

$$
H_{e f f}=H-i \pi W W^{\dagger} \text {. }
$$

This form of the effective Hamiltonian suggests that the poles of the scattering matrix have negative imaginary part and can be written as

$$
\mathcal{E}_{n}=E_{n}-\frac{i}{2} \Gamma_{n}
$$

where $E_{n}$ and $\Gamma_{n}$ are called position and width of the resonances, respectively.

\section{A.2 Wigner delay time and poles of the S-matrix}

In this section we obtain a simple formula relating the Wigner delay time with the poles of the $S$-matrix.

We start with another representation of the $S$-matrix which can be obtained from Eq. (A.13). To this end we rewrite the term containing $H_{\text {eff }}$ as follows:

$$
\begin{aligned}
W^{\dagger} \frac{1}{E-H+i \pi W W^{\dagger}} W & =W^{\dagger} \frac{1}{I+i \pi \frac{1}{E-H} W W^{\dagger}} \frac{1}{E-H} W= \\
=\sum_{k=0}^{\infty}(-i \pi)^{k}\left[W^{\dagger} \frac{1}{E-H} W\right]^{k+1} & =\frac{1}{I+i \pi W^{\dagger} \frac{1}{E-H} W} W^{\dagger} \frac{1}{E-H} W= \\
=\frac{1}{i \pi} \frac{1}{I+i K} K &
\end{aligned}
$$


where the Hermitian matrix $K$ is defined as

$$
K=\pi W^{\dagger} \frac{1}{E-H} W
$$

Now inserting the last expression of Eq. (A.16) into Eq. (A.13) we find:

$$
S=I-2 i \frac{1}{I+i K} K=\frac{(I+i K)-2 i K}{I+i K}
$$

Thus finally we obtain

$$
S=\frac{I-i K}{I+i K}
$$

According to the definition Eq. (1.9) the Wigner delay time (we put $\hbar=1$ ) is given by

$$
\tau(E)=-i \frac{\partial}{\partial E} \ln \operatorname{Det} S
$$

Using Eq. (A.19), the latter quantity can be written as

$$
\begin{aligned}
\ln \operatorname{Det} S & =\ln \frac{\operatorname{Det}(I-i K)}{\operatorname{Det}(I+i K)}=\ln \frac{\operatorname{Det}\left(I-i \pi\left[1 /(E-H) W W^{\dagger}\right]\right)}{\operatorname{Det}\left(I+i \pi\left[1 /(E-H) W W^{\dagger}\right)\right.} \\
& =\ln \frac{\operatorname{Det}\left(E-H-i \pi W W^{\dagger}\right)}{\operatorname{Det}\left(E-H+i \pi W W^{\dagger}\right)}
\end{aligned}
$$

where we used that for any matrices $U, V$ the following identity is valid:

$$
\operatorname{Det}(I-U V)=\operatorname{Det}(I-V U)
$$

Since $\ln \operatorname{Det}(X)=\operatorname{Tr} \ln (X)$ for arbitrary matrix $X$ the last expression in the Eq. (A.21) can be written as

$$
\begin{aligned}
\ln \operatorname{Det} S & =\operatorname{Tr} \ln (X)-\operatorname{Tr} \ln \left(X^{*}\right) \\
X & =E-H-i \pi W W^{\dagger}
\end{aligned}
$$

with $X^{*}$ being the complex conjugate of $X$. Taking the derivative with respect to the energy and neglecting the energy dependence of the coupling matrix $W$ (this can be done, since usually this dependence is rather smooth) we obtain

$$
\begin{aligned}
\tau(E) & =-i\left[\operatorname{Tr} \frac{d \ln (X)}{d E}-\operatorname{Tr} \frac{d \ln \left(X^{*}\right)}{d E}\right]=-i\left[\operatorname{Tr} \frac{1}{X}-\operatorname{Tr} \frac{1}{X^{*}}\right]=-2 \operatorname{Im} \operatorname{Tr} \frac{1}{X^{*}}= \\
& =-2 \operatorname{Im} \operatorname{Tr} \frac{1}{E-H+i \pi W W^{\dagger}}=-2 \operatorname{Im} \sum_{n=1}^{N} \frac{1}{E-E_{n}+i \Gamma_{n} / 2}
\end{aligned}
$$

Thus finally we obtain a simple relation connecting the Wigner delay time and the poles of the $S$-matrix:

$$
\tau(E)=\sum_{n=1}^{N} \frac{\Gamma_{n}}{\left(E-E_{n}\right)^{2}+\Gamma_{n}^{2} / 4}
$$




\section{Appendix B}

\section{Single parameter scaling hypothesis}

In this appendix we follow the ideas of Ref. [136]. Let us consider a cubic $d$-dimensional disordered sample of size $L$ with dimensionless resistance $\rho$. The exact value of $\rho$ is determined by various microscopic details like for example impurity arrangement in the sample. Therefore it is convenient to define an ensemble of samples, with different impurity arrangements, and to study the probability distribution $\mathcal{P}_{L}(\rho)$ for the resistance at the given size $L$.

When the sample size $L$ is small this distribution depends again on many details specifying the ensemble (type of impurities, their statistics and concentration, etc). However when the sample size $L$ becomes large one can expect that the microscopic details will become irrelevant and the distribution $\mathcal{P}_{L}(\rho)$ will have some universal form, which can be characterized only by a few parameters completely.

Single parameter scaling in these terms means that the large-scale distribution is specified by just one parameter $\Delta_{L}$ :

$$
\mathcal{P}_{L}(\rho)=F\left(\rho ; \Delta_{L}\right),
$$

and that this parameter obeys a scaling law:

$$
\frac{d \ln \Delta_{L}}{d \ln L}=\beta\left(\Delta_{L}\right)
$$

Thus all other parameters needed to specify the microscopic system are replaced now by a single parameter $\Delta_{L}$. This parameter scales according to universal $\beta$-function, depending only on dimension of the system.

Single parameter scaling hypothesis was proven for the case of weak disorder, under one very important assumption - the so called random phase approximation (RPA).

In order to understand the meaning of the RPA let us consider a chain composed of $n$ 
quantum resistors or scatterers. We denote by $\rho_{n-1}$ the resistance of a chain of $n-1$ scatterers and by $\rho_{0}^{(n)}$ the resistance of the last $n$th scatterer. The resistance of the $n$ scatterer chain is given by [2]

$$
\rho_{n}=\rho_{n-1}+\rho_{0}^{(n)}+2 \rho_{(n-1)} \rho_{0}^{(n)}-2\left[\rho_{(n-1)} \rho_{0}^{(n)}\left(1+\rho_{n-1}\right)\left(1+\rho_{0}^{(n)}\right)\right]^{\frac{1}{2}} \cos \theta_{n},
$$

where $\theta_{n}$ is a sum of the reflection phases associated with the scatterers $\rho_{0}^{(n)}$ and $\rho_{n-1}$. The RPA assumes that the distribution of $\theta_{n}$ is uniform. Using this assumption and the central-limit theorem one can show that the variable $u_{n}=\ln \left(1+\rho_{n}\right)$ obeys Gaussian distribution:

$$
\mathcal{P}\left(u_{n}\right) \simeq\left(2 \pi \overline{\Delta u_{n}^{2}}\right)^{-\frac{1}{2}} \exp \left[-\frac{\left(u_{n}-\bar{u}_{n}\right)^{2}}{2 \overline{\Delta u_{n}^{2}}}\right]
$$

where $\bar{u}_{n}$ and $\overline{\Delta u_{n}^{2}}$ are the mean value and the variance of the distribution of $u_{n}$ correspondingly. Thus we get two independent scaling parameters. In the weak scattering limit these two parameters become related:

$$
\overline{\Delta u_{n}^{2}}=2 \bar{u}_{n}
$$

and thus the single parameter scaling hypothesis is proved.

Returning to the distribution of the reflection phases $\mathcal{P}_{\Phi}(\Phi)$ we conclude that its deviation from uniformity can indicate the violation of the single parameter scaling hypothesis. 


\section{Appendix C}

\section{From Hamiltonian map to Fokker-Plank equation}

\section{C.1 Expression for phases in terms of Hamiltonian map}

We start with the expression for the reflection coefficient $r$ in terms of the elements of the total transfer matrix $(2.7)$ :

$$
r=-\frac{P_{11}+P_{12} \mathrm{e}^{-i k}}{P_{11}+P_{12} \mathrm{e}^{i k}}
$$

Denoting $P_{11}+P_{12} \mathrm{e}^{-i k}$ as $z \equiv \rho \mathrm{e}^{i\left(\phi+\frac{\pi}{2}\right)}$, one obtains:

$$
r=-\frac{z}{z^{*}}=\mathrm{e}^{2 i \phi}
$$

Now we find $\phi$ :

$$
\begin{aligned}
z & =P_{11}+P_{12} \cos k-i P_{12} \sin k \\
\phi+\frac{\pi}{2}=\arg (z) & =\arctan \left(\frac{-P_{12} \sin k}{P_{11}+P_{12} \cos k}\right) \\
\tan \phi & =\left(\frac{P_{11}+P_{12} \cos k}{P_{12} \sin k}\right)
\end{aligned}
$$

which leads to Eq. (2.8). Introducing the Hamiltonian map by the similarity transformation $P=R^{-1} F R$ with

$$
R=\left(\begin{array}{cc}
1 & 0 \\
\frac{\cos k}{\sin k} & \frac{-1}{\sin k}
\end{array}\right)
$$


one finds the relations between matrix elements of $P$ and matrix elements of $F$ :

$$
\begin{gathered}
\left(\begin{array}{ll}
P_{11} & P_{12} \\
P_{21} & P_{22}
\end{array}\right)=\left(\begin{array}{cc}
1 & 0 \\
\cos k & -\sin k
\end{array}\right)\left(\begin{array}{cc}
F_{11} & F_{12} \\
F_{21} & F_{22}
\end{array}\right)\left(\begin{array}{cc}
1 & 0 \\
\frac{\cos k}{\sin k} & \frac{-1}{\sin k}
\end{array}\right)= \\
\quad=\left(\begin{array}{cc}
F_{11}+F_{12} \frac{\cos k}{\sin k} & -F_{12} \frac{1}{\sin k} \\
F_{11} \cos k-F_{21} \sin k+F_{12} \frac{\cos k}{\sin k}-F_{22} \cos k & -F_{12} \frac{\cos k}{\sin k}+F_{22}
\end{array}\right)
\end{gathered}
$$

Then Eq. (C.5) yields

$$
\tan \phi=\left(\frac{F_{11}+F_{12} \frac{\cos k}{\sin k}-F_{12} \frac{1}{\sin k} \cos k}{-F_{12} \frac{1}{\sin k} \sin k}\right)=\left(\frac{F_{11}}{-F_{12}}\right)
$$

\section{C.2 Recursion relation for phases}

Dividing the third equation in (2.14) by the second one we have

$$
\begin{aligned}
\tan \theta_{n+1} & =\frac{\sin \left(\theta_{n}-k\right)-A_{n} \cos \theta_{n} \cos k}{\cos \left(\theta_{n}-k\right)-A_{n} \cos \theta_{n} \sin k}=\frac{\sin \theta_{n} \cos k-\cos \theta_{n} \sin k-A_{n} \cos \theta_{n} \cos k}{\cos \theta_{n} \cos k+\sin \theta_{n} \sin k-A_{n} \cos \theta_{n} \sin k}= \\
& =\frac{\tan \theta_{n} \cos k-\sin k-A_{n} \cos k}{\cos k+\tan \theta_{n} \sin k-A_{n} \sin k}=\frac{\tan \theta_{n}-\tan k-A_{n}}{1+\tan \theta_{n} \tan k-A_{n} \tan k}
\end{aligned}
$$

The last equation can be written as

$$
\begin{aligned}
\tan \theta_{n+1}+\tan \theta_{n+1} \tan \theta_{n} & \tan k-A_{n} \tan \theta_{n+1} \tan k=\tan \theta_{n}-\tan k-A_{n} \\
\tan \theta_{n}\left(\tan \theta_{n+1} \tan k-1\right) & =-\tan \theta_{n+1}-\tan k-A_{n}\left(1-\tan \theta_{n+1} \tan k\right) \\
\tan \theta_{n} & =\frac{\tan \theta_{n+1}+\tan k}{1-\tan \theta_{n+1} \tan k}+A_{n} \\
\tan \theta_{n} & =\tan \left(\theta_{n+1}+k\right)+A_{n}
\end{aligned}
$$

Interchanging indices $n$ and $n+1$ and taking $n=L$ in the last equation one obtains the recursion relation for the inverse map (2.15).

\section{C.3 Recursion relation for Wigner delay times}

Differentiation of the sides of Eq. (2.15) with respect to $k$ yields

$$
\frac{1}{\cos ^{2} \phi_{L+1}} \frac{d \phi_{L+1}}{d k}=\frac{1}{\cos ^{2}\left(\phi_{L}+k\right)}\left(\frac{d \phi_{L}}{d k}+1\right)-A_{L+1} \cot k
$$


Multiplying both sides of this equation by $\cos ^{2} \phi_{L+1}$ and using that $\cos ^{2} x=1 /\left(1+\tan ^{2} x\right)$ we obtain

$$
\frac{d \phi_{L+1}}{d k}=G_{L}^{-1}\left(\frac{d \phi_{L}}{d k}+1\right)-\frac{A_{L+1} \cot k}{1+\tan ^{2} \phi_{L+1}}
$$

where $G_{L}=\frac{1+\tan ^{2} \phi_{L+1}}{1+\tan ^{2}\left(\phi_{L}+k\right)}$. Using again Eq. (2.15) one can substitute

$$
\tan ^{2} \phi_{L+1}=\left(\tan \left(\phi_{L}+k\right)+A_{L+1}\right)^{2}
$$

in the last term of Eq. (C.12) and in the expression for $G$

$$
\begin{aligned}
G & =\frac{1+\left(\tan \left(\phi_{L}+k\right)+A_{L+1}\right)^{2}}{1+\tan ^{2}\left(\phi_{L}+k\right)}=1+\left(2 \tan \left(\phi_{L}+k\right) A_{L+1}+A_{L+1}^{2}\right) \cos ^{2}\left(\phi_{L}+k\right)= \\
& =1+A_{L+1} \sin \left(2\left(\phi_{L}+k\right)\right)+A_{L+1}^{2} \cos ^{2}\left(\phi_{L}+k\right)
\end{aligned}
$$

Recalling the definition of the Wigner delay time

$$
\tau_{L}=\frac{d \Phi_{L}}{d E}=2 \frac{d \phi_{L}}{d k}\left|\frac{d k}{d E}\right|=\frac{1}{\sin k} \frac{d \phi_{L}}{d k}
$$

we obtain from Eq. (C.12) the recursion relation (2.25) for the Wigner delay times.

\section{C.4 Fokker-Planck equation for distribution of Wigner delay times}

We start with the recursion relation for $\phi_{L}$ given by Eq. (2.15). Finding $\phi_{L+1}$ as a function of $\phi_{L}$ and keeping only the terms up to the second order in $A_{L+1}$ one has

$$
\begin{aligned}
\phi_{L+1} & =\arctan \left(\tan \left(\phi_{L}+k\right)+A_{L+1}\right)= \\
& =\phi_{L}+k+\frac{1}{1+\tan ^{2}\left(\phi_{L}+k\right)} A_{L+1}+\frac{1}{2} \frac{-2 \tan \left(\phi_{L}+k\right)}{\left(1+\tan ^{2}\left(\phi_{L}+k\right)\right)^{2}} A_{L+1}^{2}+\cdots= \\
& =\phi_{L}+k+\cos ^{2}\left(\phi_{L}+k\right) A_{L+1}-\tan \left(\phi_{L}+k\right) \cos ^{4}\left(\phi_{L}+k\right) A_{L+1}^{2}
\end{aligned}
$$

Replacing the random variable $A_{L+1}^{2}$ by its variance $\sigma_{A}^{2}$ and going to the continuum limit we obtain a stochastic differential equation for the random variable $\phi(2.26)$.

In order to get a stochastic differential equation for the delay times we first multiply both sides of Eq. (2.25) by $\sigma_{V}^{2}$ and introduce the rescaled variable $\tilde{\tau}=\sigma_{V}^{2} \tau$. Keeping again only the terms up to the second order in $A_{L+1}$ one has

$$
\begin{aligned}
\tilde{\tau}_{L+1} & =G_{L}^{-1}\left(\tilde{\tau}_{L}+\frac{\sigma_{V}^{2}}{\sin k}\right)+\cdots= \\
& =\left(1-A_{L+1} \sin \left(2\left(\phi_{L}+k\right)\right)-A_{L+1}^{2}\left(\cos ^{2}\left(\phi_{L}+k\right)-\sin ^{2}\left(\phi_{L}+k\right)\right)+\cdots\right)\left(\tilde{\tau}_{L}+\frac{\sigma_{V}^{2}}{\sin k}\right)= \\
& =\tilde{\tau}_{L}-A_{L+1} \sin \left(2\left(\phi_{L}+k\right)\right) \tilde{\tau}_{L}-A_{L+1}^{2}\left(\cos ^{2}\left(\phi_{L}+k\right)-\sin ^{2}\left(2\left(\phi_{L}+k\right)\right)\right) \tilde{\tau}_{L}+\sigma_{A}^{2} \sin k
\end{aligned}
$$


Repeating the same procedure described below Eq. (C.16) we obtain a stochastic differential equation for the random variable $\tilde{\tau}(2.26)$.

The next step is to write the Fokker-Planck equation for the joint probability distribution $\mathcal{P}(\phi, \tilde{\tau}, L)$ which corresponds to the system of two stochastic differential equation (2.26) [61]

$$
\begin{aligned}
\frac{\mathcal{P}(\phi, \tilde{\tau}, L)}{\partial L}= & -\frac{\partial}{\partial \phi}\left(k-\sigma_{A}^{2} \sin (\phi+k) \cos ^{3}(\phi+k)\right)- \\
& -\frac{\partial}{\partial \tilde{\tau}}\left(-\sigma_{A}^{2}\left(\tilde{\tau}\left(\cos ^{2}(\phi+k)-\sin ^{2} 2(\phi+k)\right)-\sin k\right)\right)+ \\
& +\frac{\sigma_{A}^{2}}{2}\left[\frac{\partial}{\partial \phi}\left(\left(\cos ^{2}(\phi+k)\right)\left(\frac{\partial}{\partial \phi}\left(\cos ^{2}(\phi+k)\right)+\frac{\partial}{\partial \tilde{\tau}}(\tilde{\tau} \sin 2(\phi+k))\right)\right)+\right. \\
& \left.+\frac{\partial}{\partial \tilde{\tau}}\left((\tilde{\tau} \sin 2(\phi+k))\left(\frac{\partial}{\partial \phi}\left(\cos ^{2}(\phi+k)\right)+\frac{\partial}{\partial \tilde{\tau}}(\tilde{\tau} \sin 2(\phi+k))\right)\right)\right]
\end{aligned}
$$

Using the fact that $\phi$ follows the uniform distribution and assuming that the variables $\phi$ and $\tilde{\tau}$ are statistically independent, one can integrate Eq. (C.18) over $\phi$ and obtain the Fokker-Planck equation (2.27) for $\mathcal{P}_{\tilde{\tau}}(\tilde{\tau})$. 


\section{Appendix D}

\section{Mean free path and diffusion coefficient for the kicked rotor model}

\section{D.1 Calculation of the mean free path}

Let us consider the evolution operator of the two-dimensional kicked rotor introduced in Eq. (3.6) (to simplify the calculations we put $T=1$ )

$$
U=e^{-i H_{0}\left(\mathcal{L}_{1}, \mathcal{L}_{2}\right) / 2} e^{-i V\left(\theta_{1}, \theta_{2}\right)} e^{-i H_{0}\left(\mathcal{L}_{1}, \mathcal{L}_{2}\right) / 2} .
$$

The set of the orthogonal eigenfunctions of the free Hamiltonian $H_{0}$ is given by plane waves in the angle representation

$$
\phi_{\left(n_{1}, n_{2}\right)}\left(\theta_{1}, \theta_{2}\right)=\frac{1}{2 \pi} e^{i\left(n_{1} \theta_{1}+n_{2} \theta_{2}\right)} .
$$

They are normalized in such a way that

$$
\int_{0}^{2 \pi} \int_{0}^{2 \pi} d \theta_{1} d \theta_{2}\left|\phi_{\left(n_{1}, n_{2}\right)}\left(\theta_{1}, \theta_{2}\right)\right|^{2}=1
$$

Let us denote by $U_{\left(k_{1}, k_{2}\right),\left(n_{1}, n_{2}\right)}$ the matrix elements of the evolution operator in this basis

$$
U_{\left(k_{1}, k_{2}\right),\left(n_{1}, n_{2}\right)}=\left\langle\phi_{\left(k_{1}, k_{2}\right)}|U| \phi_{\left(n_{1}, n_{2}\right)}\right\rangle .
$$

According to the definition of the evolution operator the modulus square of its elements have the meaning of the probability to change the initial momentum $\left(n_{1}, n_{2}\right)$ to the final momentum $\left(k_{1}, k_{2}\right)$ in one kick. Therefore one can define the mean free path in momentum space [97] by

$$
l_{M}^{2}=\sum_{r_{1}} \sum_{r_{2}}\left(r_{1}^{2}+r_{2}^{2}\right)\left|U_{(0,0),\left(r_{1}, r_{2}\right)}\right|^{2}
$$


Here we used the fact that $\left|U_{\left(k_{1}+m_{1}, k_{2}+m_{2}\right),\left(n_{1}+m_{1}, n_{2}+m_{2}\right)}\right|^{2}=\left|U_{\left(k_{1}, k_{2}\right),\left(n_{1}, n_{2}\right)}\right|^{2}$, so without loss of generality one can take the initial momentum equal to $(0,0)$. In order to calculate the right hand side of Eq. (D.5) we first give an explicit expression to the matrix elements $U_{\left(k_{1}, k_{2}\right),\left(n_{1}, n_{2}\right)}$ :

$$
\begin{aligned}
& U_{\left(k_{1}, k_{2}\right),\left(n_{1}, n_{2}\right)}= \\
& \quad=\sum_{i, j, s, t}\left\langle\phi_{\left(k_{1}, k_{2}\right)}\left|e^{-i H_{0}\left(\mathcal{L}_{1}, \mathcal{L}_{2}\right) / 2}\right| \phi_{(i, j)}\right\rangle\left\langle\phi_{(i, j)}\left|e^{-i V\left(\theta_{1}, \theta_{2}\right)}\right| \phi_{(s, t)}\right\rangle\left\langle\phi_{(s, t)}\left|e^{-i H_{0}\left(\mathcal{L}_{1}, \mathcal{L}_{2}\right) / 2}\right| \phi_{\left(n_{1}, n_{2}\right)}\right\rangle= \\
& =e^{-i\left(H_{0}\left(k_{1}, k_{2}\right)+H_{0}\left(n_{1}, n_{2}\right)\right) / 2}\left\langle\phi_{\left(k_{1}, k_{2}\right)}\left|e^{-i V\left(\theta_{1}, \theta_{2}\right)}\right| \phi_{\left(n_{1}, n_{2}\right)}\right\rangle= \\
& =e^{-i\left(H_{0}\left(k_{1}, k_{2}\right)+H_{0}\left(n_{1}, n_{2}\right)\right) / 2} \frac{1}{4 \pi^{2}} \int_{0}^{2 \pi} \int_{0}^{2 \pi} d \theta_{1} d \theta_{2} e^{-i V\left(\theta_{1}, \theta_{2}\right)} e^{i\left(n_{1}-k_{1}\right) \theta_{1}+i\left(n_{2}-k_{2}\right) \theta_{2}}
\end{aligned}
$$

Taking $\left(k_{1}, k_{2}\right)=(0,0)$ and $\left(n_{1}, n_{2}\right)=\left(r_{1}, r_{2}\right)$ we obtain

$$
U_{(0,0),\left(r_{1}, r_{2}\right)}=e^{-i\left(H_{0}(0,0)+H_{0}\left(r_{1}, r_{2}\right)\right) / 2} \frac{1}{4 \pi^{2}} \int_{0}^{2 \pi} \int_{0}^{2 \pi} d \theta_{1} d \theta_{2} e^{-i V\left(\theta_{1}, \theta_{2}\right)} e^{i r_{1} \theta_{1}} e^{i r_{2} \theta_{2}}
$$

The substitution of this expression into Eq. (D.5) yields

$l_{M}^{2}=\sum_{r_{1}} \sum_{r_{2}}\left(r_{1}^{2}+r_{2}^{2}\right) \frac{1}{\left(4 \pi^{2}\right)^{2}} \int_{0}^{2 \pi} \int_{0}^{2 \pi} \int_{0}^{2 \pi} \int_{0}^{2 \pi} d \theta_{1} d \theta_{2} d \tilde{\theta}_{1} d \tilde{\theta}_{2} e^{-i\left(V\left(\theta_{1}, \theta_{2}\right)-V\left(\tilde{\theta}_{1}, \tilde{\theta}_{2}\right)\right)} e^{i r_{1}\left(\theta_{1}-\tilde{\theta}_{1}\right)} e^{i r_{2}\left(\theta_{2}-\tilde{\theta}_{2}\right)}$

Taking into account that

$$
r_{1}^{2} e^{i r_{1}\left(\theta_{1}-\tilde{\left.\theta 1_{1}\right)}\right.}=\frac{\partial^{2}}{\partial \theta_{1} \partial \tilde{\theta}_{1}} e^{i r_{1}\left(\theta_{1}-\tilde{\theta} 1_{1}\right)}
$$

and that the same is valid for $r_{2}$ the partial integration of the Eq. (D.8) gives

$$
\begin{aligned}
l_{M}^{2}= & \sum_{r_{1}} \sum_{r_{2}} \frac{1}{\left(4 \pi^{2}\right)^{2}} \int_{0}^{2 \pi} \int_{0}^{2 \pi} \int_{0}^{2 \pi} \int_{0}^{2 \pi} d \theta_{1} d \theta_{2} d \tilde{\theta}_{1} d \tilde{\theta}_{2}\left(\frac{\partial^{2}}{\partial \theta_{1} \partial \tilde{\theta}_{1}}+\frac{\partial^{2}}{\partial \theta_{2} \partial \tilde{\theta}_{2}}\right) \\
& e^{-i\left(V\left(\theta_{1}, \theta_{2}\right)-V\left(\tilde{\theta}_{1}, \tilde{\theta}_{2}\right)\right)} e^{i r_{1}\left(\theta_{1}-\tilde{\theta}_{1}\right)} e^{i r_{2}\left(\theta_{2}-\tilde{\theta}_{2}\right)} .
\end{aligned}
$$

The summation of the exponents over $r_{1}$ and $r_{2}$ yields two $\delta$-functions:

$$
\begin{aligned}
l_{M}^{2}= & \frac{1}{4 \pi^{2}} \int_{0}^{2 \pi} \int_{0}^{2 \pi} \int_{0}^{2 \pi} \int_{0}^{2 \pi} d \theta_{1} d \theta_{2} d \tilde{\theta}_{1} d \tilde{\theta}_{2}\left(\frac{\partial^{2}}{\partial \theta_{1} \partial \tilde{\theta}_{1}}+\frac{\partial^{2}}{\partial \theta_{2} \partial \tilde{\theta}_{2}}\right) \\
& e^{-i\left(V\left(\theta_{1}, \theta_{2}\right)-V\left(\tilde{\theta}_{1}, \tilde{\theta}_{2}\right)\right)} \delta\left(\theta_{1}-\tilde{\theta}_{1}\right) \delta\left(\theta_{2}-\tilde{\theta}_{2}\right)= \\
= & \frac{1}{4 \pi^{2}} \int_{0}^{2 \pi} \int_{0}^{2 \pi} \int_{0}^{2 \pi} \int_{0}^{2 \pi} d \theta_{1} d \theta_{2} d \tilde{\theta}_{1} d \tilde{\theta}_{2}\left(\frac{\partial V}{\partial \theta_{1}}\left(\theta_{1}, \theta_{2}\right) \frac{\partial V}{\partial \tilde{\theta}_{1}}\left(\tilde{\theta}_{1}, \tilde{\theta}_{2}\right)+\frac{\partial V}{\partial \theta_{2}}\left(\theta_{1}, \theta_{2}\right) \frac{\partial V}{\partial \tilde{\theta}_{2}}\left(\tilde{\theta}_{1}, \tilde{\theta}_{2}\right)\right) \\
& e^{-i\left(V\left(\theta_{1}, \theta_{2}\right)-V\left(\tilde{\theta}_{1}, \tilde{\theta}_{2}\right)\right)} \delta\left(\theta_{1}-\tilde{\theta}_{1}\right) \delta\left(\theta_{2}-\tilde{\theta}_{2}\right)= \\
= & \frac{1}{4 \pi^{2}} \int_{0}^{2 \pi} \int_{0}^{2 \pi} d \theta_{1} d \theta_{2}\left[\left(\frac{\partial V}{\partial \theta_{1}}\left(\theta_{1}, \theta_{2}\right)\right)^{2}+\left(\frac{\partial V}{\partial \theta_{2}}\left(\theta_{1}, \theta_{2}\right)\right)^{2}\right]
\end{aligned}
$$


The last expression can be written in a compact form

$$
l_{M}^{2}=\frac{1}{4 \pi^{2}} \int_{0}^{2 \pi} \int_{0}^{2 \pi} d \theta_{1} d \theta_{2}\left\|\vec{\nabla} V\left(\theta_{1}, \theta_{2}\right)\right\|^{2}
$$

Now we calculate the mean free path in the case where the potential $V\left(\theta_{1}, \theta_{2}\right)$ is given by Eq. (3.3):

$$
V\left(\theta_{1}, \theta_{2}\right)=k\left(\cos \left(\theta_{1}\right) \cos \left(\theta_{2}\right) \cos (\alpha)+\frac{1}{2} \sin \left(2 \theta_{1}\right) \cos \left(2 \theta_{2}\right) \sin (\alpha)\right)
$$

Taking the derivative of this expression with respect to $\theta_{1}$ and $\theta_{2}$ one has

$$
\begin{aligned}
\left(\frac{\partial V}{\partial \theta_{1}}\left(\theta_{1}, \theta_{2}\right)\right)^{2}= & k^{2}\left(\sin ^{2} \theta_{1} \cos ^{2} \theta_{2} \cos ^{2} \alpha+\cos ^{2} 2 \theta_{1} \cos ^{2} 2 \theta_{2} \sin ^{2} \alpha-\right. \\
& \left.-2 \sin \theta_{1} \cos 2 \theta_{1} \cos \theta_{2} \cos 2 \theta_{2} \cos \alpha \sin \alpha\right) \\
\left(\frac{\partial V}{\partial \theta_{2}}\left(\theta_{1}, \theta_{2}\right)\right)^{2}= & k^{2}\left(\cos ^{2} \theta_{1} \sin ^{2} \theta_{2} \cos ^{2} \alpha+\sin ^{2} 2 \theta_{1} \sin ^{2} 2 \theta_{2} \sin ^{2} \alpha+\right. \\
& \left.+2 \cos \theta_{1} \sin 2 \theta_{1} \sin \theta_{2} \sin 2 \theta_{2} \cos \alpha \sin \alpha\right)
\end{aligned}
$$

The integration over $\theta_{1}$ and $\theta_{2}$ yields

$$
l_{M}^{2}=\frac{1}{4 \pi^{2}} k^{2}\left(\pi^{2} \cos ^{2} \alpha+\pi^{2} \sin ^{2} \alpha+\pi^{2} \cos ^{2} \alpha+\pi^{2} \sin ^{2} \alpha\right)=\frac{k^{2}}{2}
$$

The analogous calculations for the one-dimensional kicked rotor yield

$$
l_{M}^{2}=\frac{1}{2 \pi} \int_{0}^{2 \pi} d \theta\left(\frac{\partial V}{\partial \theta}(\theta)\right)^{2}
$$

Inserting into this expression the potential $V(\theta)$ of the one-dimensional kicked rotor

$$
V(\theta)=k\left(\cos (\theta) \cos (\alpha)+\frac{1}{2} \sin (2 \theta) \sin (\alpha)\right)
$$

we find that the mean free path is given by exactly the same formula as in the Eq.(D.15):

$$
l_{M}=\frac{k}{\sqrt{2}}
$$

Thus the mean free path is in our model completely determined by the kicking strength. 


\section{D.2 Diffusion coefficient in the random phase approx- imation}

We start by writing the classical maps Eq. (3.4) for the general form of the potential $V\left(\theta_{1}, \theta_{2}\right)$ :

$$
\begin{aligned}
\theta_{1}(n+1)= & \theta_{1}(n)+\tau_{1} T \mathcal{L}_{1}(n) \bmod 2 \pi \\
\theta_{2}(n+1)= & \theta_{2}(n)+\tau_{2} T \mathcal{L}_{2}(n) \bmod 2 \pi \\
\mathcal{L}_{1}(n+1)= & \mathcal{L}_{1}(n)-\frac{\partial V}{\partial \theta_{1}}\left(\theta_{1}(n+1), \theta_{2}(n+1)\right) \\
\mathcal{L}_{2}(n+1)= & \mathcal{L}_{2}(n)-\frac{\partial V}{\partial \theta_{2}}\left(\theta_{1}(n+1), \theta_{2}(n+1)\right) \\
& \left.-\sin \left(2 \theta_{1}(n+1)\right) \sin \left(2 \theta_{2}(n+1)\right) \sin (\alpha)\right)
\end{aligned}
$$

The diffusion coefficient in momentum space is defined as

$$
D=\lim _{t \rightarrow \infty} \frac{\left\langle\mathcal{L}_{1}^{2}(t)+\mathcal{L}_{2}^{2}(t)\right\rangle}{t}
$$

The average in this expression is taken over an ensemble of trajectories, with different initial conditions. Using the classical maps for $\mathcal{L}_{1}$ and $\mathcal{L}_{2}$ we obtain

$$
\begin{array}{r}
D=\lim _{n \rightarrow \infty} \frac{1}{n T}\left[\sum_{i=1}^{n}\left\langle\left(\frac{\partial V}{\partial \theta_{1}}\left(\theta_{1}(i), \theta_{2}(i)\right)\right)^{2}+\left(\frac{\partial V}{\partial \theta_{2}}\left(\theta_{1}(i), \theta_{2}(i)\right)\right)^{2}\right\rangle+\right. \\
\left.+\sum_{i=1}^{n} \sum_{j=1, j \neq i}^{n}\left\langle\frac{\partial V}{\partial \theta_{1}}\left(\theta_{1}(i), \theta_{2}(i)\right) \frac{\partial V}{\partial \theta_{1}}\left(\theta_{1}(j), \theta_{2}(j)\right)+\frac{\partial V}{\partial \theta_{2}}\left(\theta_{1}(i), \theta_{2}(i)\right) \frac{\partial V}{\partial \theta_{2}}\left(\theta_{1}(j), \theta_{2}(j)\right)\right\rangle\right]
\end{array}
$$

For the large values of the kicking strength $k$, in a good approximation one can consider the phases $\theta_{1}(i)$ and $\theta_{2}(i)$ as random variables which are uncorrelated for different $i$ and distributed uniformly in the interval $[0,2 \pi]$. Using this random phase approximation it is easily to show that only the diagonal terms in Eq. (D.21) give non-zero contribution in the limit $n \rightarrow \infty$. Taking into account that the distribution of the phases is uniform one can convert the sum for the diagonal terms into an integral. Finally we obtain

$$
D=\frac{1}{4 \pi^{2} T} \int_{0}^{2 \pi} \int_{0}^{2 \pi} d \theta_{1} d \theta_{2}\left[\left(\frac{\partial V}{\partial \theta_{1}}\left(\theta_{1}, \theta_{2}\right)\right)^{2}+\left(\frac{\partial V}{\partial \theta_{2}}\left(\theta_{1}, \theta_{2}\right)\right)^{2}\right]
$$

Then the formula for the diffusion coefficient measured in number of kicks $D_{k}=T D$ is given by

$$
D_{k}=\frac{1}{4 \pi^{2}} \int_{0}^{2 \pi} \int_{0}^{2 \pi} d \theta_{1} d \theta_{2}\left\|\vec{\nabla} V\left(\theta_{1}, \theta_{2}\right)\right\|^{2}
$$


which has exactly the same form as one appearing Eq. (D.12). Thus we obtain that in the random phase approximation the following relation between the mean free path and the diffusion coefficient is valid:

$$
D_{k}=l_{M}^{2}=\frac{k^{2}}{2}
$$

Therefore changing the kicking strength we can easy tune the diffusion constant or Thouless conductance (for disordered systems). This allows us to investigate various regimes: ballistic, diffusive and localized. 


\section{Appendix E}

\section{Construction of the $S$-matrix for the kicked rotor model}

In Ref. [99] Fyodorov and Sommers suggested the following construction of the $S$-matrix for systems with discrete time dynamics (maps):

$$
S(\omega)=\sqrt{I-\tau^{\dagger} \tau}-\tau^{\dagger} \frac{1}{e^{-i \omega}-A} U \tau
$$

where $U$ is a unitary matrix describing the evolution of the closed system, $\tau$ is a rectangular $N \times M$ diagonal matrix with entries $\tau_{i j}=\delta_{i j} \tau_{i}, 1 \leq i \leq N, 1 \leq j \leq M, 0 \leq \tau_{i} \leq 1$. This matrix provides a coupling to the "outside world" that makes the system open. The corresponding evolution of the open system is described by the subunitary operator $A=$ $U \sqrt{I-\tau \tau^{\dagger}}$.

For the kicked rotor model it is natural to change this construction in such a way that the dynamics of the open system is given by the operator $A=\sqrt{I-W^{\dagger} W} U$ (first evolution of the closed system and then "the elimination of the particles which go out"). Having this in mind, one can rewrite the definition of the $S$-matrix as follows:

$$
S(\omega)=\sqrt{I-W W^{\dagger}}-W U \frac{1}{e^{-i \omega}-A} W^{\dagger} .
$$

In the case of the perfect coupling, $W$ is a rectangular $M \times N$ matrix with entries $W_{i j}=$ $\delta_{i j}, 1 \leq i \leq M, 1 \leq j \leq N$. Then $W W^{\dagger}=I_{M \times M}$ and $W^{\dagger} W$ is a $N \times N$ diagonal matrix with $M$ non-zero elements equal to one. So $P \equiv I-W^{\dagger} W$ is a projection operator that implies:

$$
\sqrt{I-W^{\dagger} W}=I-W^{\dagger} W
$$

Taking this into account, the expression (E.2) can be simplified:

$$
S(\omega)=-W U \frac{1}{e^{-i \omega}-\left(I-W^{\dagger} W\right) U} W^{\dagger}=W U \frac{1}{U-e^{-i \omega}-W^{\dagger} W U} W^{\dagger} .
$$


Using the unitarity of the evolution operator $U$ we can rewrite the last expression as follows:

$$
S=W \frac{1}{I-W^{\dagger} W-U^{\dagger} e^{-i \omega}} W^{\dagger}
$$

This equation is useful for the derivation of the Wigner-Smith operator and we will use it later.

Below we prove that the $S$-matrix constructed in this way is unitary.

\section{E.1 Unitarity of the $S$-matrix}

Let us rewrite the expression for $S$-matrix (E.4) in a more symmetric way. To this end we use a series expansion for inverse operator in (E.4):

$$
\begin{gathered}
{\left[U-e^{-i \omega}-W^{\dagger} W U\right]^{-1}=\left[\left(U-e^{-i \omega}\right)\left(I-\frac{1}{U-e^{-i \omega}} W^{\dagger} W U\right)\right]^{-1}=} \\
=\left[I-\frac{1}{U-e^{-i \omega}} W^{\dagger} W U\right]^{-1} \frac{1}{U-e^{-i \omega}}=\sum_{k \geq 0}\left(\frac{1}{U-e^{-i \omega}} W^{\dagger} W U\right)^{k} \frac{1}{U-e^{-i \omega}} .
\end{gathered}
$$

Substituting this expansion in Eq. (E.4) we obtain

$$
\begin{aligned}
S & =\sum_{k \geq 0} W U\left(\frac{1}{U-e^{-i \omega}} W^{\dagger} W U\right)^{k} \frac{1}{U-e^{-i \omega}} W^{\dagger}=\sum_{k \geq 0}\left(W U \frac{1}{U-e^{-i \omega}} W^{\dagger}\right)^{k+1}= \\
& =\frac{W U \frac{1}{U-e^{-i \omega}} W^{\dagger}}{I-W U \frac{1}{U-e^{-i \omega}} W^{\dagger}}=\frac{W \frac{U}{U-e^{-i \omega}} W^{\dagger}}{W\left(I-\frac{U}{U-e^{-i \omega}}\right) W^{\dagger}}=-\frac{W \frac{U}{U-e^{-i \omega}} W^{\dagger}}{W \frac{e^{-i \omega}}{U-e^{-i \omega}} W^{\dagger}}
\end{aligned}
$$

Now using the unitarity of the evolution operator $U$ we can calculate the Hermitian conjugate $S$-matrix:

$$
S^{\dagger}=-\frac{W \frac{U^{-1}}{U^{-1}-e^{i \omega}} W^{\dagger}}{W \frac{e^{i \omega}}{U^{-1}-e^{i \omega}} W^{\dagger}}=-\frac{W \frac{e^{-i \omega}}{e^{-i \omega}-U} W^{\dagger}}{W \frac{U}{e^{-i \omega}-U} W^{\dagger}}=-\frac{W \frac{e^{-i \omega}}{U-e^{-i \omega}} W^{\dagger}}{W \frac{U}{U-e^{-i \omega}} W^{\dagger}}=S^{-1} .
$$

Thus the unitarity of the $S$-matrix is proven.

\section{E.2 Wigner-Smith operator}

The Wigner-Smith operator generalizes the notion of Wigner delay time. It is defined as (for the kicked rotor model one should use quasi-energy instead of energy in the definition of Wigner-Smith operator)

$$
Q=\frac{1}{i} S^{\dagger} \frac{\partial S}{\partial \omega}
$$


Introducing a new operator

$$
K \equiv \frac{1}{I-W^{\dagger} W-U^{\dagger} e^{-i \omega}}
$$

and taking the derivative of both sides of Eq. (E.5) we obtain

$$
\begin{aligned}
\frac{\partial S}{\partial \omega} & =W \frac{\partial K}{\partial \omega} W^{\dagger}=W \frac{1}{I-W^{\dagger} W-U^{\dagger} e^{-i \omega}}(-i) e^{-i \omega} U^{\dagger} \frac{1}{I-W^{\dagger} W-U^{\dagger} e^{-i \omega}} W^{\dagger}= \\
& =-i e^{-i \omega} W K U^{\dagger} K W^{\dagger}
\end{aligned}
$$

Then the definition of the Wigner-Smith yields

$$
Q=-i e^{-i \omega} W K^{\dagger} W^{\dagger} W K U^{\dagger} K W^{\dagger}
$$

The trace of the above expression gives the value of the Wigner delay time allowing to calculate it without using numerical differentiation. 


\section{Bibliography}

[1] Y. Imry, Introduction to Mesoscopic Physics (Oxford University Press, New York, 1998).

[2] S. Datta, Electronic Transport in Mesoscopic Systems (Cambridge University Press, Cambridge, 1995).

[3] M. L. Mehta, Random Matrices (Academic, New York, 1991).

[4] C. W. J. Beenakker, Rev. Mod. Phys. 69, 731 (1997).

[5] R. Landauer, IBM Journ. Res. Development 1, 223 (1957).

[6] R. Landauer, Philos. Mag, 21, 863 (1970).

[7] C. Mahaux and H. A. Weidenmüller, Shell Model Approach in Nuclear Reactions (North-Holland, Amsterdam, 1969).

[8] Y. V. Fyodorov and H.-J. Sommers, J. Math. Phys. 38, 1918 (1997).

[9] v. I. Kukulin, V. M. Krasnopol'sky, and J. Horacek, Theory of Resonances (Kluwer Academic Publisher, Dordrecht, 1989).

[10] R. A. Jalabert, A. D. Stone, and Y. Alhassid, Phes. Rev. Lett. 68, 3468 (1992).

[11] I. L. Aleiner, P. W. Brouwer, and L. I. Glazman, Phys. Rep. 358, 309 (2002).

[12] D. S. Wiersma, M. P. V. Albada, and A. Lagendijk, Nature (London) 373, 203 (1995).

[13] A. L. Burin, M. A. Ratner, H. Cao, and S. H. Chang, Phys. Rev. Lett. 88, 093904 (2002).

[14] L. Eisenbud, Ph. D. Thesis, Princeton University, unpublished, 1948.

[15] E. P. Wigner, Phys. Rev. 98, 145 (1955).

[16] C. A. A. de Carvalho and H. M. Nussenzveig, Phys. Rep. 364, 83 (2002). 
[17] F. T. Smith, Phys. Rev. 118, 349 (1960).

[18] M. Büttiker, A. Prêtre, and H. Thomas, Phys. Rev. Lett. 70, 4114 (1993).

[19] M. H. Pedersen, S. A. van Langen, and M. Büttiker, Phys. Rev. B 57, 1838 (1998).

[20] S. Pilgram and M. Büttiker, Phys. Rev. Lett. 89, 200401 (2002).

[21] V. A. Gopar, P. A. Mello, and M. Büttiker, Phys. Rev. Lett. 77, 3005 (1996).

[22] C. W. J. Beenakker and P. W. Brouwer, Physica E 9, 463 (2001).

[23] A. D. Mirlin, Phys. Rep. 326, 259 (2000).

[24] E. P. Wigner, Ann. Math. 53, 36 (1951).

[25] F. J. Dyson, J. Math. Phys. 3, 140 (1962).

[26] O. Bohigas, M. J. Giannoni, and C. Schmit, Phys. Rev. Lett. 52, 1 (1984).

[27] M. C. Gutzwiller, Chaos in Classical and Quantum Mechanics (Springer-Verlag, New York, 1991).

[28] F. Haake, Quantum Signatures of Chaos, Vol. 54 of Springer Series in Synergetics (Springer-Verlag, Berlin, 1991), .

[29] H.-J. Stöckmann, Quantum Chaos. An Introduction (Cambridge University Press, Cambridge, 1999).

[30] A. V. Andreev, O. Agam, B. D. Simons, and B. L. Altshuler, Phys. Rev. Lett. 76, 3947 (1996).

[31] A. Altland and M. R. Zirnbauer, Phys. Rev. Lett. 77, 4536 (1996).

[32] K. Richter and M. Sieber, Phys. Rev. Lett. 89, 206801 (2002).

[33] G. Berkolaiko, H. Schanz, and R. S. Whitney, Phys. Rev. Lett. 88 (2002) 10410188 , 104101 (2002).

[34] L. P. Gor'kov and G. M. Eliashberg, Sov. Phys. JETP 21, 940 (1965).

[35] K. B. Efetov, Adv. Phys. 32, 53 (1983).

[36] K. B. Efetov, Supersymmetry in Disorder and Chaos (Cambridge University Press, Cambridge, 1997).

[37] P. W. Anderson, Phys. Rev. 109, 1492 (1958).

[38] A. Lee and T. V. Ramakrishnan, Rev. Mod. Phys. 57, 287 (1985). 
[39] A. Ossipov, T. Kottos, and T. Geisel, Phys. Rev. B 61, 11411 (2000).

[40] G. Casati, B. Chirikov, J. Ford, and F. M. Izrailev, Lect. Notes Phys. 93, 334 (1979).

[41] F. M. Izrailev, Phys. Rep. 196, 299 (1990).

[42] A. Ossipov, T. Kottos, and T. Geisel, Phys. Rev. E 65, 055209(R) (2002).

[43] A. Ossipov, T. Kottos, and T. Geisel, accepted by Europhys. Lett., 2003.

[44] F. Steinbach, A. Ossipov, T. Kottos, and T. Geisel, Phys. Rev. Lett. 85, 4426 (2000).

[45] A. Ossipov, M. Weiss, T. Kottos, and T. Geisel, Phys. Rev. B 64, 224210 (2001).

[46] T. Geisel, R. Ketzmerick, and G. Petschel, in Quantum Chaos: Between Order and Disorder, Eds. J. Casati and B. Chirikov (Cambridge University Press, Cambridge, 1995), S. 633.

[47] N. F. Mott and W. D. Twose, Adv. Phys. 10, 107 (1961).

[48] R. E. Borland, Proc. R. Soc. London Ser. A 274, 529 (1963).

[49] H. Frustenberg, Trans. Ann. Math. Soc. 108, 377 (1963).

[50] N. W. Ashcroft and N. D. Mermin, Solid State Physics (Saunders College Publishing, Florida, 1976).

[51] F. M. Izrailev, T. Kottos, and G. P. Tsironis, Phys. Rev. B 52, 3274 (1995).

[52] F. M. Izrailev, S. Ruffo, and L. Tessieri, J. Phys. A 31, 5263 (1998).

[53] C. Barnes and J. M. Luck, J. Phys. A 23, 1717 (1990).

[54] H. G. Schuster, Deterministic Chaos. An Introduction (Physik-Verlag, Weinheim, 1984).

[55] A. D. Stone, D. C. Allan, and J. D. Joannopoulos, Phys. Rev. B 27, 836 (1983).

[56] S. K. Joshi, A. K. Gupta, and A. M. Jayannavar, Phys. Rev. B 58, 1092 (1998).

[57] P. L. Sulem, Physica (Amsterdam) 70, 190 (1973).

[58] R. Kree and A. Schmid, Z. Phys. B 42, 297 (1981).

[59] J. Heinrichs, J. Phys.: Condens. Matter 2, 1559 (1990).

[60] L. I. Deych, A. A. Lisyansky, and B. L. Altshuler, Phys. Rev. Lett. 84, 2678 (2000).

[61] C. W. Gardiner, Handbook of Stochastic Methods (Springer-Verlag, Berlin, 1985). 
[62] A. M. Jayannavar, G. V. Vijayagovindan, and N. Kumar, Z. Phys. B 75, 77 (1989).

[63] C. J. Bolton-Heaton, C. J. Lambert, V. I. Falko, V. Prigodin, and A. J. Epstein, Phys. Rev. B 60, 10569 (1999).

[64] A. Comtet and C. Texier, J. Phys. A 30, 8017 (1997).

[65] C. Texier and A. Comtet, Phys. Rev. Lett. 82, 4220 (1999).

[66] S. A. Ramakrishna and N. Kumar, Phys. Rev. B 61, 1363 (2000).

[67] J. Heinrichs, Phys. Rev. B 65, 075112 (2002).

[68] G. Casati, G. Maspero, and D. Shepelyansky, Phys. Rev. Lett. 82, 524 (1999).

[69] M. Titov and Y. V. Fyodorov, Phys. Rev. B 61, R2444 (2000).

[70] M. Terraneo and I. Guarneri, Eur. Phys. J. B 18, 303 (2000).

[71] A. J. Lichtenberg and M. A. Lieberman, Regular and Chaotic Dynamics (SpringerVerlag, Berlin, 1992).

[72] B. L. Altshuler, V. E. Kravtsov, and I. V. Lerner, in Mesoscopic Phenomena in Solids, Eds. J. Casati and B. Chirikov (North Holland, Amsterdam, 1991), S. 449.

[73] B. A. Muzykantskii and D. E. Khmelnitiskii, Phys. Rev. B 51, 5480 (1995).

[74] Y. V. Fyodorov and A. D. Mirlin, Int. J. Mod. Phys. B 8, 3795 (1994).

[75] Y. V. Fyodorov and A. D. Mirlin, Phys. Rev. B 51, 13403 (1995).

[76] V. I. Falko and K. B. Efetov, Europhys. Lett. 32, 627 (1995).

[77] V. I. Falko and K. B. Efetov, Phys. Rev. B 52, 17413 (1995).

[78] L. E. Reichl, The Transition to Chaos (Springer-Verlag, New York, 1992).

[79] S. Fishman, D. R. Grempel, and R. E. Prange, Phys. Rev. Lett. 49, 509 (1982).

[80] A. D. Mirlin, J. Math. Phys. 38, 1888 (1997).

[81] A. D. Mirlin, in International School of Physics 'Enrico Fermi' Course CXLIII "New Directions in Quantum Chaos", Eds. G.Casati, I. Guarneri, and U. Smilansky (IOS Press, Amsterdam, 2000).

[82] I. E. Smolyarenko and B. L. Altshuler, Phys. Rev. B 55, 10451 (1997).

[83] U. Meirav, M. A. Kastner, and S. J. Wild, Phys. Rev. Lett. 65, 771 (1990). 
[84] V. Uski, B. Mehlig, R. A. Römer, and M. Schreiber, Phys. Rev. B 62, 7699(R) (2000).

[85] V. Uski, B. Mehlig, and M. Schreiber, Phys. Rev. B 63, 241101(R) (2001).

[86] B. Nikolić, Phys. Rev. B 64, 014203 (2001).

[87] V. Zelevinsky, B. A. Brown, N. Frazier, and M. Horoi, Phys. Rep. 276, 85 (1996).

[88] A. F. Brunello, T. Uzer, and D. Farrelly, Phys. Rev, Lett. 76, 2874 (1996).

[89] G. Benenti, G. Casati, and D. L. Shepelyansky, Phys. Rev. A 56, 3297 (1997).

[90] H.-J. Stöckmann and J. Stein, Phys. Rev. Lett. 64, 2215 (1990).

[91] S. Sridhar, Phys. Rev. Lett. 67, 785 (1991).

[92] A. Kudrolli, V. Kidambi, and S. Sridhar, Phys. Rev. Lett. 75, 822 (1995).

[93] P. Pradhan and S. Sridhar, Phys. Rev. Lett. 85, 2360 (2000).

[94] J. U. Nockel and A. D. Stone, Nature 385, 45 (1997).

[95] R. Blümel and U. Smilansky, Phys. Rev. Lett. 69, 217 (1992).

[96] G. Casati, R. Graham, I.Guarneri, and F. Izrailev, Phys. Lett. 190, 159 (1994).

[97] E. Doron and S. Fishman, Phys. Rev. Lett. 60, 867 (1988).

[98] F. Borgonovi, I. Guarneri, and D. Shepelyansky, Phys. Rev. A 43, 4517 (1991).

[99] Y. Fydorov and H.-J. Sommers, JETP Lett. 72, 422 (2000).

[100] V. M. Apalkov, M. E. Raikh, and B. Shapiro, Phys. Rev. Lett. 89, 126601 (2002).

[101] J.-P. Bouchaud and A. Georges, Phys. Rep. 195, 127 (1990).

[102] P. W. Brouwer, K. M. Frahm, and C. W. J. Beenakker, Phys. Rev. Lett. 78, 4737 (1997).

[103] H.-J. Sommers, D. V. Savin, and V. V. Sokolov, Phys. Rev. Lett. 87, 094101 (2001).

[104] H. Schomerus, K. M. Frahm, M. Patra, and C. W. J. Beenakker, Physica A 278, 469 (2000).

[105] B. Mehlig and J. T. Chalker, J. Math. Phys. 41, 3233 (2000).

[106] T. Kottos and M. Weiss, Phys. Rev. Lett. 89, 056401 (2002).

[107] R. Peierls, Z. Phys. 80, 763 (1933). 
[108] L. Onsager, Phil. Mag. 43, 1006 (1952).

[109] J. Zak, Phys. Rev. 168, 686 (1968).

[110] H.-J. Schellnhuber and G. Obermair, Phys. Rev. Lett. 45, 276 (1980).

[111] P. G. Harper, Proc. Roy. Soc. London A 68, 874 (1955).

[112] R. Ketzmerick, Dissertation, Frankfurt, 1992.

[113] Z. M. Stadnik, Physical Properties of Quasicrystals (Springer-Verlag, Berlin, 1999).

[114] D. Shechtman, I. Blech, D. Gratias, and J. V. Cahn, Phys. Rev. Lett. 53, 1951 (1984).

[115] D. Levine and J. P. Steinhardt, Phys. Rev. Lett. 53, 2477 (1984).

[116] Quasicrystals, Eds. J.-B. Suck, M. Schreiber, and P. Häussler (Springer-Verlag, Berlin, 2002).

[117] M. Kohmoto, L. P. Kadanoff, and G. Tang, Phys. Rev. Lett. 50, 1870 (1983).

[118] S. Ostlund, R. Pandit, D. Rand, H. J. Schellnhuber, and E. D. Siggia, Phys. Rev. Lett. 50, 1873 (1983).

[119] J. M. Luck and D. Petritis, J. Stat. Phys. 42, 289 (1986).

[120] J. Bellisard, B. Iochum, E. Scoppola, and D. Testard, Comm. Math. Phys. 125, 527 (1989).

[121] R. Merlin, K. Bajema, R. Clarke, F.-Y. Yuang, and P. K. Bhatacharaya, Phys. Rev. Lett. 55, 1768 (1985).

[122] T. Schlösser, K. Ensslin, J. P. Kotthaus, and M. Holland, Europhys. Lett. 33, 683 (1996).

[123] I. N. Harris, H. D. M. Davies, J. F. Ryan, A. J. Turberfield, C. T. Foxon, and J. J. Harris, Europhys. Lett. 29, 333 (1995).

[124] U. Kuhl and H.-J. Stöckmann, Phys. Rev. Lett. 80, 3232 (1998).

[125] S. Aubry and G. Andre, Ann. Israel Phys. Soc. 3, 133 (1980).

[126] J. B. Sokolov, Phys. Rep. 126, 189 (1985).

[127] T. Geisel, R. Ketzmerick, and G. Petschel, Phys. Rev. Lett. 66, 1651 (1991).

[128] Y. Last, Commun. Math. Phys. 164, 421 (1994).

[129] R. Ketzmerick, K. Kruse, F. Steinbach, and T. Geisel, Phys. Rev. B 58, 9881 (1998). 
[130] D. R. Hofstadter, Phys. Rev. B 14, 2239 (1976).

[131] K. Kruse, Dissertation, Frankfurt, 1998.

[132] F. M. Dittes, H. L. Harney, and A. Müller, Phys. Rev. A 45, 701 (1992).

[133] W. H. Press and S. A. Teukolsky, Numerical Recipes in Fortran 90 (Cambridge University Press, Cambridge, 1996).

[134] M. Dittes, Phys. Rep. 339, 215 (2000).

[135] R. Blümel and U. Smilansky, Phys. Rev. Lett. 60, 477 (1988).

[136] A. Cohen, Y. Roth, and B. Shapiro, Phys. Rev. B 38, 12125 (1988). 


\section{Acknowledgments}

I would like to thank Prof. Dr. Theo Geisel for giving me the opportunity to stay in his group after finishing my diploma thesis and for his support during all this time. Due to his efforts outstanding working conditions and a stimulating environment have been created in the group.

I would also like to express my deepest gratitude to Dr. Tsampikos Kottos for his permanent guidance and advice. Without his fruitful ideas and criticism, his readiness for discussions and enthusiastic support this thesis would not appear.

Special thanks are extended to Dmitri Bibitchkov, Matthias Kaschube and Michael Schnabel for various inspiring discussions of scientific as well as nonscientific character.

I am grateful to all the members of the group for a friendly atmosphere: Steffen Arnrich, Rita Bartels, Oliver Bendix, Kai Broeking, Dr. Wolf-Dieter Brandt, Dirk Brockmann, Michael Buschermöhle, Michael Denker, Markus Diesmann, Dr. Silke Dodel, Dr. Udo Ernst, Dr. Ragnar Fleischmann, Dr. Denny Fliegner, Sven Goedeke, Dr. Michael Herrmann, Moritz Hiller, Dr. Lars Hufnagel, Katharina Jeremias, Dr. Roland Ketzmerick, Dr. Norbert Mayer, Abigail Morrison, Björn Naundorf, Gerhard Nolte, Dr. Marc-Felix Otto, Felix Petri, Mathias Puhlmann, Dr. Holger Schanz, Dr. Dennis Springsguth, Dr. Frank Steinbach, Yorck-Fabian Temme, Tom Tetzlaff, Dr. Marc Timme, Corinna Trautsch, Dr. Matthias Weiß, Dr. Fred Wolf, Regina Wunderlich, and Alexander Zumdieck. 


\section{Lebenslauf}

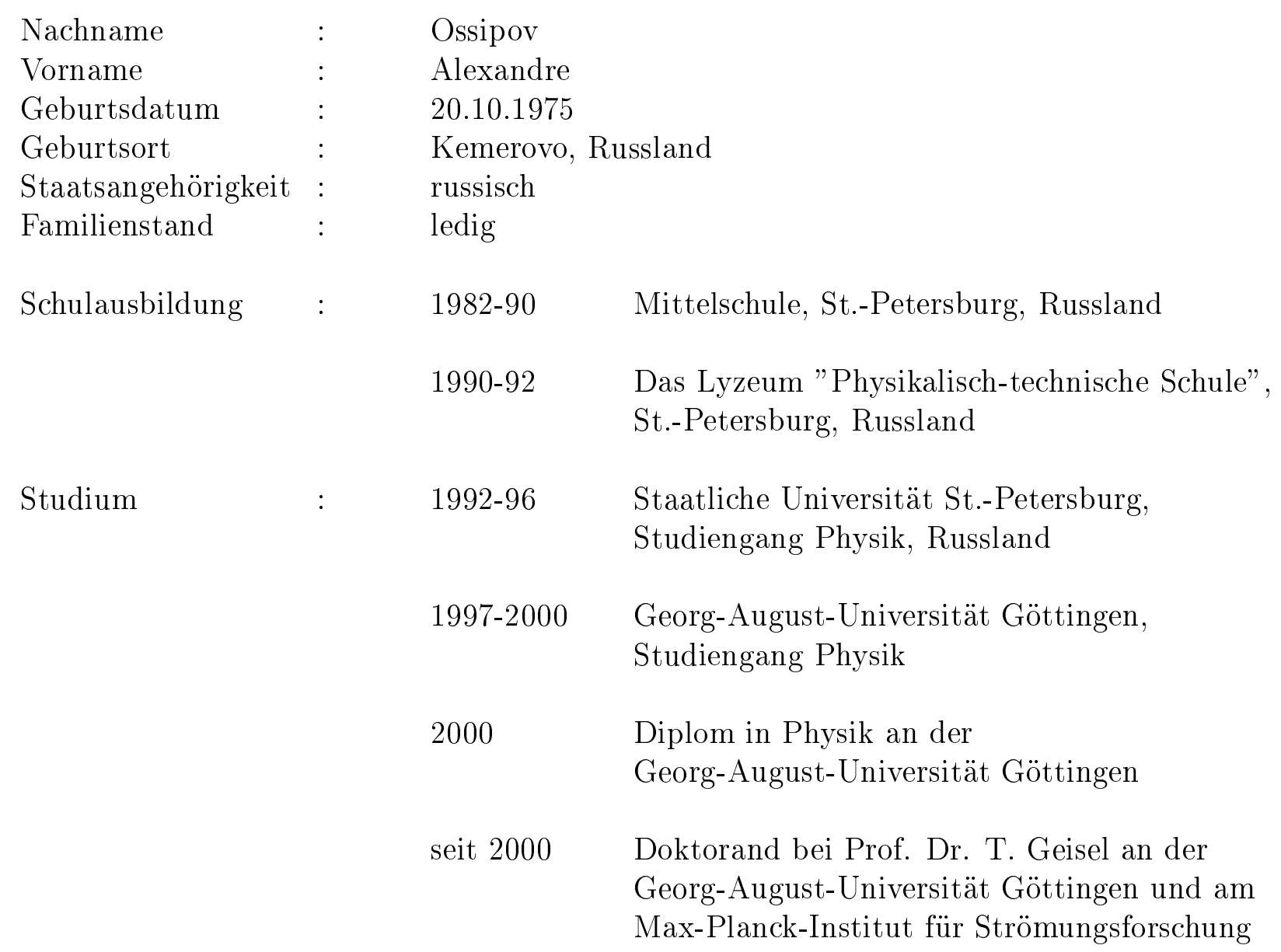

